University of Louisville

ThinkIR: The University of Louisville's Institutional Repository

Electronic Theses and Dissertations

$5-2018$

\title{
The involvement of epithelial cells in arenavirus-induced pathogenesis.
}

Nikole Leslie Margaret Warner

University of Louisville

Follow this and additional works at: https://ir.library.louisville.edu/etd

Part of the Virology Commons

\section{Recommended Citation}

Warner, Nikole Leslie Margaret, "The involvement of epithelial cells in arenavirus-induced pathogenesis." (2018). Electronic Theses and Dissertations. Paper 2964.

https://doi.org/10.18297/etd/2964

This Doctoral Dissertation is brought to you for free and open access by ThinkIR: The University of Louisville's Institutional Repository. It has been accepted for inclusion in Electronic Theses and Dissertations by an authorized administrator of ThinkIR: The University of Louisville's Institutional Repository. This title appears here courtesy of the author, who has retained all other copyrights. For more information, please contact thinkir@louisville.edu. 


\title{
THE INVOLVEMENT OF EPITHELIAL CELLS IN ARENAVIRUS-INDUCED PATHOGENESIS
}

\author{
By \\ Nikole Leslie Margaret Warner \\ B.S., Marian University, 2010 \\ M.S., University of Louisville, 2014
}

\author{
A Dissertation \\ Submitted to the Faculty of the \\ School of Medicine of the University of Louisville \\ In Partial Fulfillment of the Requirements \\ for the Degree of
}

Doctor of Philosophy in Microbiology and Immunology

\author{
Department of Microbiology and Immunology \\ University of Louisville \\ Louisville, KY
}

May 2018 
Copyright 2018 by Nikole Leslie Margaret Warner

All rights reserved 



\title{
THE INVOLVEMENT OF EPITHELIAL CELLS IN ARENAVIRUS-INDUCED PATHOGENESIS
}

\author{
By \\ Nikole Leslie Margaret Warner \\ B.S., Marian University, 2010 \\ M.S., University of Louisville, 2014
}

A Dissertation Approved on

April 16, 2018

By the Following Dissertation Committee Members:

Igor Lukashevich, Ph.D.

(Dissertation Director)

Kevin Sokoloski, Ph.D.

(Co-Advisor)

Juliane Beier, Ph.D.

Donghoon Chung, Ph.D.

Michele Kosiewicz, Ph.D.

Thomas Mitchell, Ph.D. 


\section{DEDICATION}

This dissertation is dedicated to my late mother, whose love and support has yet to be rivaled. To my father, who taught me that hard work, grit, and dedication are priceless qualities to have. To my brother, Kris, who is always there to bring

me back down to Earth. And to my love, John, for being my best friend and rock when I needed it most. I love you all, more than I can express. 


\section{ACKNOWLEDGEMENTS}

I would like to first thank Dr. Igor Lukashevich, for giving me the opportunity to join the lab and taking a chance on me. I had little experience before graduate school, but he has allowed me to learn and to grow the last 5.5 years as a person and a scientist. Thank you for allowing me the opportunity to work with you.

To Dr. Kevin Sokoloski; you have been a beacon of light in dark times for me. Thank you for taking me under your wing, for your guidance, and reminding me time and time again, not to sweat the small stuff.

Thank you to Dr. Hoon Chung, for always making me feel welcome to ask questions, no matter how silly, and for being an outstanding lab neighbor. Your continued encouragement and humor has been greatly appreciated the last 5 years.

I am grateful for such a wonderful committee, Dr. Tom Mitchell, Dr. Juliane Beier, and Dr. Michele Kosiewicz, for your support and guidance time and time again. Your encouragement and direction have meant so much to me, and has allowed me to grow over the years of my training. All three of you are not only incredible scientists, but you are some of the best people I have met. Thank you for caring for me and guiding me through my graduate school career.

A special thank you to the Department of Microbiology and Immunology faculty, staff, and students. Thank you for taking a chance on me, for providing me life-long friendships, and being a place where I could be myself. This experience was highlighted by the love and support of this department.

To Marlene and the CPM staff; thank you for doing the most incredible job running the facilities at UofL. You are some of the most hardworking people I have ever met, and I could not have finished my degree without the proper facilities. Thank you for teaching me, and keeping our facilities safe and running smooth.

Thank you to the past and present members of the Lukashevich Lab. Jenny, you have been my lab mom for 5 years and I could not have done it without your support and advice. You have been through it all with me, and I truly appreciate all you do for our lab. Dylan, we did not have a lot of time together, but best of luck to you in the future of pursuing this wild ride. To my past 
members Dr. Min Wang, who was essential for our second publication and provided several figures of Chapter 3 , and to Patrick Whang, who was a friend, colleague, and teacher my early years in the lab, and continues to be my friend today.

Thank you to Gretchen Holz. It is hard to put into words what you have meant to me through this experience. We have been through a roller coaster of emotions, successes, failures, losses, and wins, but through it all, you have been there. Thank you for being the best lab mate anyone could ask for; for listening to my hairbrained ideas, the woes of my life, and especially for the continued laughs that we shared. Thank you for our late-night lab dates, and for being like a big sister to me. You are truly, an incredible woman and your friendship is one of the best things to have come from this experience.

Thank you to Mr. Scott and Drewbie who have been incredible lab neighbors. Scott, I will truly miss our kitty cat talks and deep conversations that we've had through the years. Thank you for being one of the kindest, most down to Earth people I have had the pleasure of calling my friend. Drew, thank you for always being my chatterbox, lunch partner, and streak of positivity that I have needed the last 3 years. You are a great scientist, and I'm very proud of you for how far you've come. I can't wait to see where your future takes you.

A special thanks to my M\&l friends who have been essential in my journey. Juanita, you have been such a sweet and wonderful addition to my life. Thank you for always being there to lend an ear or give a hug. The laughs we have shared, have fueled me through this trip. I can't wait to meet Kai and continue our friendship. James, thank you for your encouragement and support and being a wonderful running partner. To Bethany, Ashley, Tiva, Amanda, Sarah, Snake, and Chris; thank you for the endless laughs, jokes, and conversations we have shared. You have gotten me through the last 6 years, and I couldn't have done it without you.

To the Louisvirology members, Autumn, Claire, and Trey. Thank you for sharing Kevin, and always being there to listen and laugh with me when I needed it most.

To my beautiful and funny friends that I have made throughout my life who continually supported me on this journey; Kelly, Ashley, Emily, and Amanda. To Abbey, who is the whole reason I decided to take on this adventure. And thank you to my incredibly supportive and silly family.

Thank you to Daddy, for being the most supportive, incredible person I have ever met. You have taught me that nothing is too hard to accomplish, to do what I love and what I wanted to do, and enjoy life as much as I can. Thank you for teaching me how to work hard, grind when I need to, and laugh every minute I 
can. Thank you for pushing me and reminding me: DLTBW. I love you more than words can express.

Thank you to Momma, who taught me what unconditional love meant. Thank you for being the most loving, silly person I ever knew. I miss you every day and I wish you could be here, but I know you would be proud.

To my little Bekah, who I could not thank enough for being a wonderful partner for Kris. You are an amazing, strong, intelligent woman and I love you like a little sister. I'm so proud of you and I'll be here for you through your graduate school experience, every step of the way.

Thank you to my bubby, Kris. Thank you for bringing me back down to reality when I'm on the anxiety wagon, for listening to my problems, but for most of all, always knowing how to make me laugh. You are my best friend and I love you with all my heart. I'm so proud of you.

And finally, thank you to my love, John. You have brought such light to my life the last 3 years. It's hard to put into words what you mean to me, but you and Kuzco have gotten me through some of the toughest times in my life. You have always been my biggest fan and loved me, no matter how hangry I got, and for that, thank you. I love you my sweets, and I could not have finished this without you. 


\title{
ABSTRACT \\ THE INVOLVEMENT OF EPITHELIAL IN ARENAVIRUS-INDUCED PATHOGENESIS
}

\author{
Nikole Leslie Margaret Warner
}

April 16, 2018

Mammalian Arenaviruses are a geographically and genetically diverse family of viruses, which is separated into two sub-groups; the Old World (OW) and New World (NW) groups. Of the OW viruses, Lassa virus (LASV), found endemically in Western Africa, is an important human pathogen, causing hundreds of thousands of infections, and several thousand deaths annually. Interestingly, some villages in endemic regions, up to $45 \%$ of the population show seropositivity for the virus. It is hypothesized that seropositivity is a result of natural infection through inhalation or ingestion of infectious particles. However, the exact mechanism is still unknown.

LASV's natural reservoir is Mastomys natalensis, a common rat found in sub-Saharan Africa. Epidemiological studies have identified the inhalation, and/or ingestion of infectious rodent excreta as the primary route of transmission from rodent reservoir to human hosts. Additionally, controlled experiments investigating intragastric (i.g.) versus intravenous (i.v.) routes of inoculation of non-human primates (NHPs) have continued transmission through these routes. 
These studies utilized Lymphocytic Choriomeningitis Virus (LCMV)-WE, a strain of LCMV that results in Lassa Fever (LF)-like disease in NHPs, and LCMVArmstrong (ARM), a strain of LCMV that mimics subclinical infection. When administered i.v., LCMV-WE-infected NHPs became systemically infected, showing clinical signs much like that of LF, and died. However, when orally infected with this virus through i.g. inoculation, some of these animals recovered, and later, were protected from lethal doses of i.v. WE challenge.

Due to the nature of natural transmission from rodent to humans, epithelial cells are amongst the first cells to come in contact with the virus. However, the role(s) of the epithelial barrier during these infections have yet to be investigated. In order to investigate the role of these cells during arenaviral infection, here, a cell culture model was developed to investigate the interaction of OW mammalian arenaviruses at the site of intragastric inoculation. An important finding of this works is that the patterns of entry and release are viral dependent, and attachment to epithelial surfaces may play a role in these phenomena. Furthermore, regardless of their pathogenic potential in NHPs, both strains of LCMV, as well as LASV's close relative, MOPV, showed similar patterns of entry and release when exposed to the apical and basolateral surfaces of polarized intestinal epithelia. Additionally, the replication patters of vaccine candidate ML29; a reassortant virus that contains the $L$ segment of MOPV, and $S$ segment of LASV, providing the exact same GP1 of LASV, were characterized. Interestingly, ML-29 virus entered and released in a different pattern than was observed with LCMV and MOPV. 
To determine if patterns of viral entry and release were driven by attachment differences, LCMV, MOPV, and ML-29 viral attachment to the surface of polarized epithelia was analyzed. LCMV an MOPV attaches to the apical and basolateral surfaces of the cells with similar efficiency. However, ML-29 showed decreased attachment to the basolateral surface of these cells as compared to the apical surface.

Due to differences in pathogenicity seen in NHPs infected i.v. with LCMVWE and ARM, we hypothesized that these viruses would show differences in entry and release patterns in the polarized Caco-2 cells. However, these viruses replicated in much the same way. From these observations, we sought to further investigate differences in viral replication that may explain pathogenic differences between these closely related viruses. To do so, we investigated intracellular trafficking under the hypothesis that it may be responsible for these differences.

Through the use of chemical inhibitors and immunofluorescence with confocal microscopy, a number of differences through the intracellular trafficking of LCMV-ARM and WE. The data indicates that LCMV-WE bypasses the TLR-2 receptor interaction in early endosome, and does not produce an IL-6 response in infected macrophages, opposed to LCMV-ARM-infected cells. Additionally, co-staining with LCMV and late endosome marker RAB7, showed more colocalization with LCMV-ARM than that of LCMV-WE. Furthermore, when blocking acidification of late endosome/lysosome with bafilomycin treatments, LCMV-ARM was more sensitive to $\mathrm{pH}$ change in the late endosome, indicating 
that fusion occurs at less acidic conditions. These less acidic conditions promote earlier release at viral RNA in the case of LCMV-ARM versus that of WE.

Together, these results signify differences in viral replication are tissue and viral specific. Furthermore, this research provides a platform to continue investigating key differences in viral replication between viruses of close genetic relatedness. 


\section{TABLE OF CONTENTS}

DEDICATION

ACKNOWLEDGEMENTS ………………………………...................................... iv

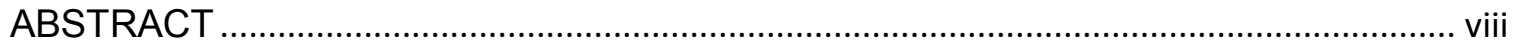

LIST OF FIGURES ...............................................................................................ii

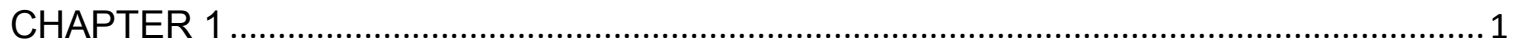

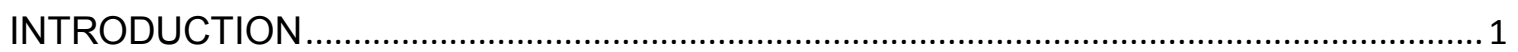

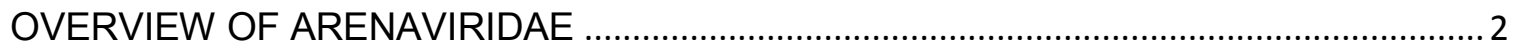

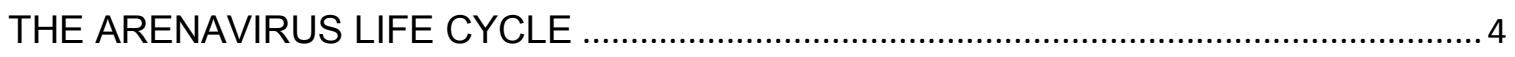

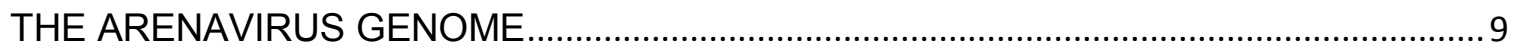

INDIVIDUAL VIRUSES OF MEDICAL CONCERN..........................................................

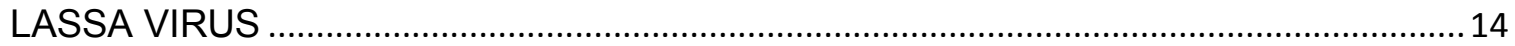

LYMPHOCYTIC CHORIOMENINGITIS VIRUS …………............................................

TRANSMISSION OF RODENT-BORN ARENAVIRUSES TO HUMANS ………..............20

IMMUNE RESPONSE TO OW ARENAVIRAL .................................................................

ANIMAL MODELS MIMICKING NATURAL TRANSMISSION …………………………...25

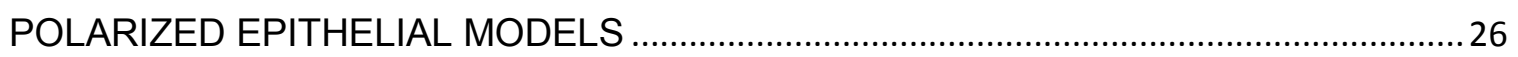

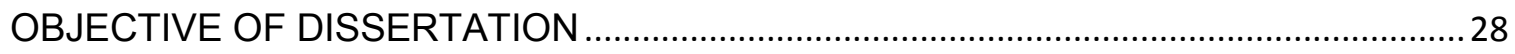

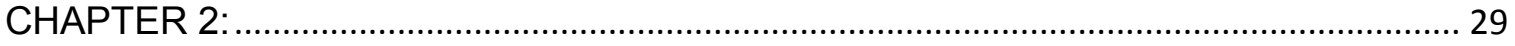

THE INTERACTION OF MAMMALIAN ARENAVIRUSES WITH POLARIZED

EPITHELIAL CELLS ……………………………………………………………….... 29

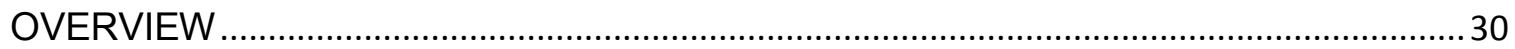

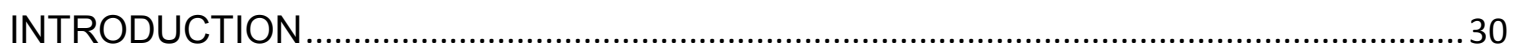

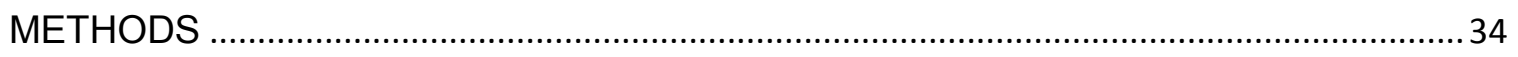

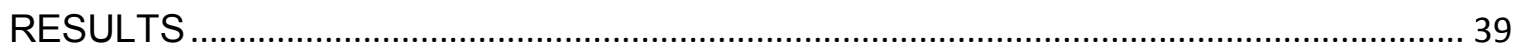

DISCUSSION

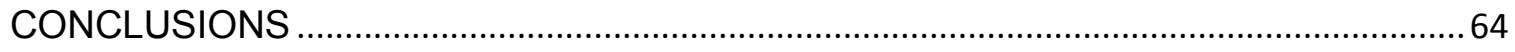

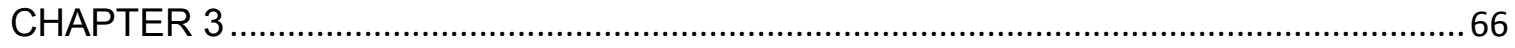

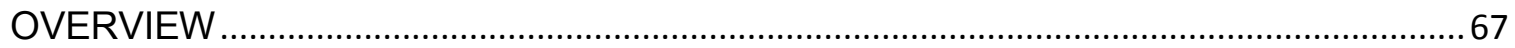

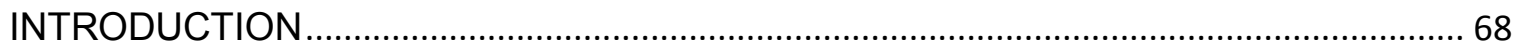

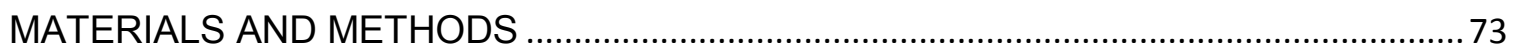

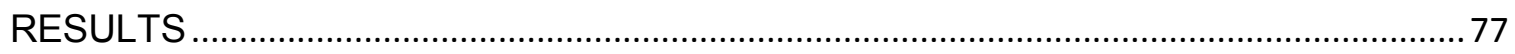

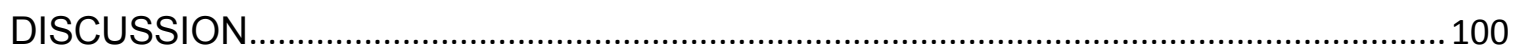




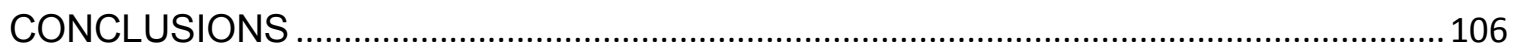

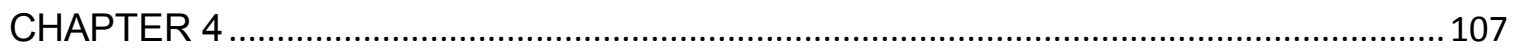

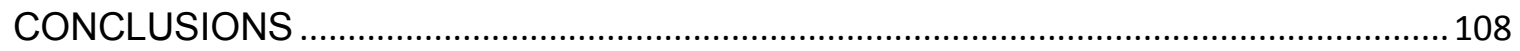

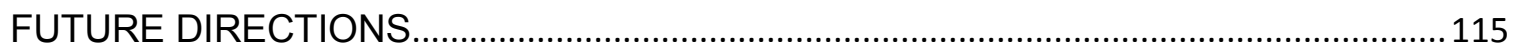

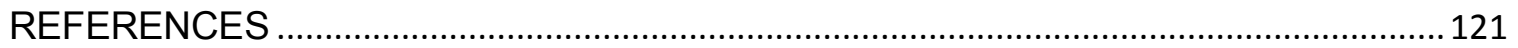

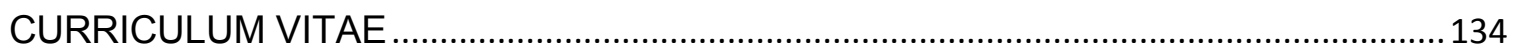




\section{CHAPTER 1}

\section{LIST OF FIGURES}

Figure 1. Taxonomy of Mammarenaviruses. ................................................... 3

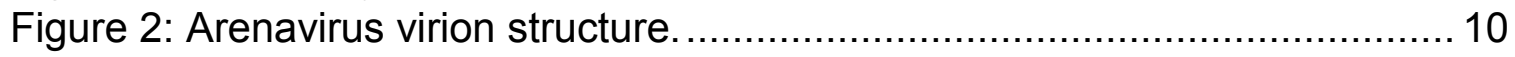

Figure 3. Arenavirus Genome................................................................... 11

\section{CHAPTER 2}

Figure 4 LCMV-Armstrong does not replicate well in polarized human bronchial

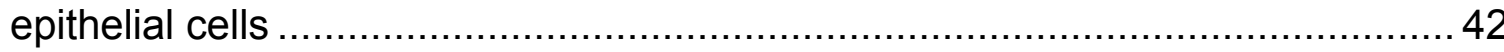

Figure 5. LCMV-ARM, but not WE is impacted by polarization of MDCK cells ... 44

Figure 6. MDCK cells are not permissive to LCMV-Armstrong ......................... 45

Figure 7. Old World (OW) mammarenaviruses do not alter integrity of model

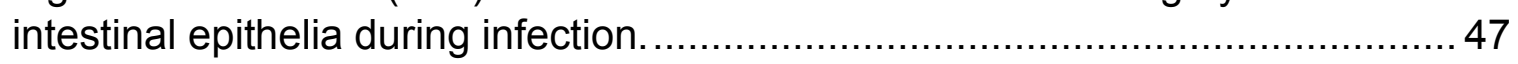

Figure 8 Polarization of Caco-2 cells does not significantly impact OW arenaviral replication

Figure 9 LCMV-Armstrong and LCMV-WE show similar patterns of entry and release in polarized Caco-2 cells regardless of pathogenic differences. ............51 Figure 10. ML-29 replication in polarized Caco-2 cells differ from the replication patterns of LCMV.

Figure 11. Mopeia virus replication in polarized Caco-2 cells follows a similar pattern as LCMV replication.

Figure 12. Summary of entry and exit patterns of OW arenaviruses in polarized Caco-2 cells 56

Figure 13 Attachment of ML-29 on the basolateral surface of polarized Caco-2 cells is significantly lower than apical attachment.

\section{CHAPTER 3}

Figure 14. TLR2 siRNA transfection down regulates IL-6 in LCMV-ARM-infected murine macrophages.

Figure 15. LCMV-ARM has strong co-localization patterns of LCMV NP with EEA-1 and TLR-2 markers.

Figure 16. LCMV-ARM shows more co-localization with RAB-7 marker, compared to LCMV-WE.

Figure 18. LCMV-WE has significant co-staining patterns with IRAK-1 compared to LCMV-ARM.

Figure 22. LCMV-ARM and LCMV-WE replication is inhibited by depletion of membrane cholesterol, but not by microtubule disruption in non-polarized Caco-2 cells.

Figure 24. Blocking of late endosome acidification results in a decrease in the percentage of viral infection in both LCMV-ARM and LCMV-WE in a strainspecific manner. 
CHAPTER 1

INTRODUCTION 


\section{OVERVIEW OF ARENAVIRIDAE}

Arenaviridae is a family of viruses that are known to cause asymptomatic, chronic infections of rodents, and human disease ranging from asymptomatic infection to deadly hemorrhagic fever. These viruses have been identified worldwide, utilizing rodents as the host reservoir. Arenaviridae gets its name from the term arenosus which is Latin for "sandy" due to their appearance in electron microscope sections, caused by ribosomes obtained from their infected host cells [1]. When these viruses are transmitted to humans, they can cause minor to severe infection that may lead to hemorrhagic fevers. Others seem to be nonpathogenic for humans, or rarely found in human hosts.

Initially, arenaviruses were thought to only infect mammals, but recently, arenaviruses have been identified as the causative agent of inclusion body disease in the boid family of snakes [2]. These findings spurred a change in arenaviral taxonomy, leading to a new genus of arenaviruses; mammarenavirus, which utilize a mammalian host (Figure 1), and of genus reptarenavirus which target reptilian hosts [3]. Among the mammarenavirus genus, there is further division into the Old World (OW) or LCMV-Lassa virus complex, and the New World (NW), or Tacaribe virus (TCRV) complex $[3,4]$. The mammarenaviruses are separated into the OW and NW complexes based on geographical location and genetic relatedness. The OW complex has a single lineage containing five species; Lassa virus (LASV), Mopeia virus (MOPV), Ippy virus, 


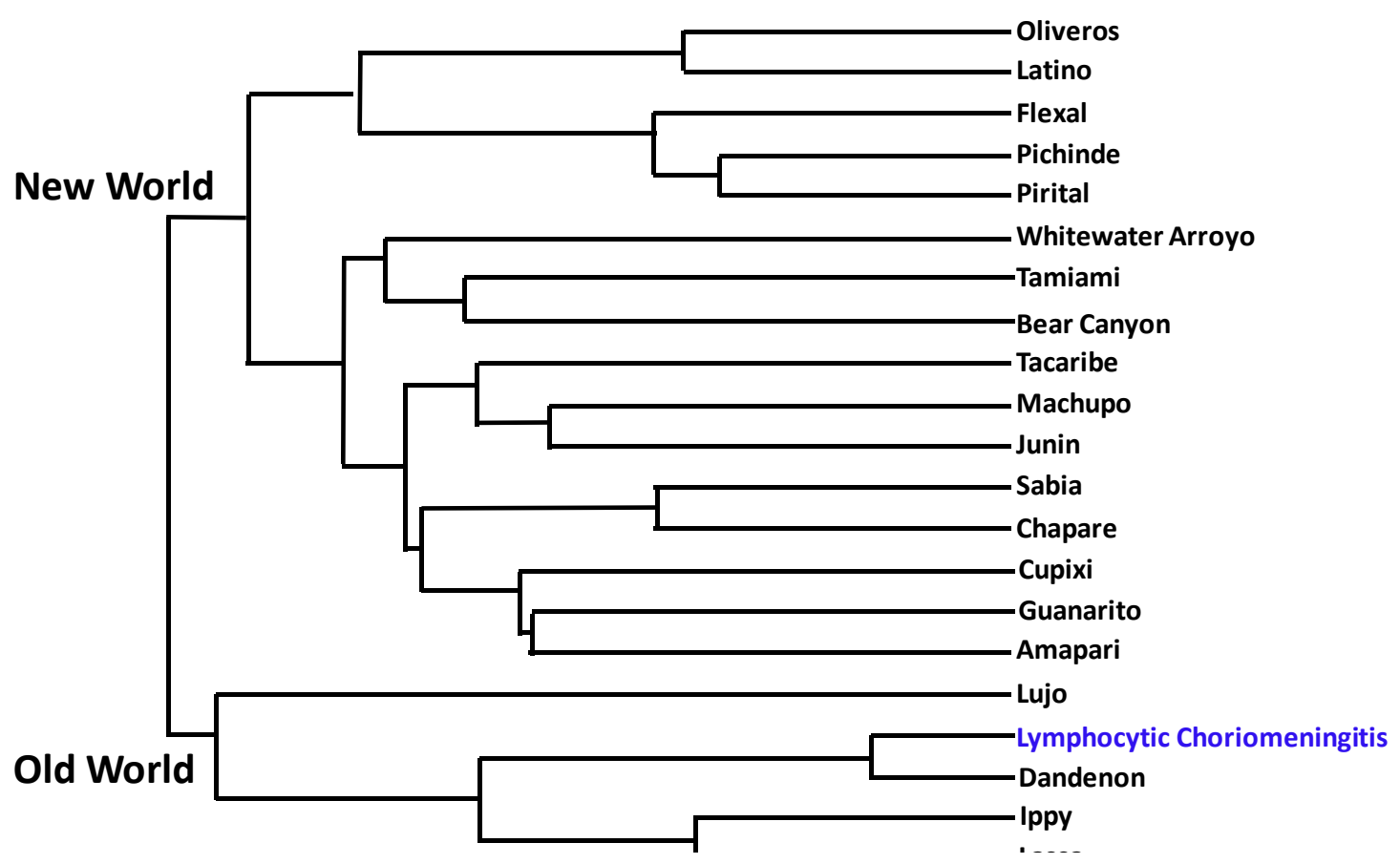

\section{Figure 1. Taxonomy of Mammarenaviruses.}

Multiple sequence alignment of the complete GPC from different arenaviruses were analyzed via CLUSTALW analysis. A phylogenetic tree was generated using MacVector 12.6.0. Hoizontal distances represent protein differences. Viruses highlighted in blue represent viruses used in studies presented here. Image adapted from McLay et. al 2014 [5]. 
Mobala virus, and Lymphocytic Choriomeningitis virus (LCMV), as well as some newly discovered viruses including Lujo, Morogoro, Dandenong, and Kodoko virus [6-8]. The NW complex has several lineages; clade A, B, C, and A/Rec. Clade A of the NW complex contains 5 species; Flexal mammarenavirus, Parana mammarenavirus, Pichinde mammarenavirus, Pirital mammarenavirus, and Allpahuayo mammarenavirus. Most of the NW viruses are not known to be pathogenic for humans, and are located in South America. Arenaviruses of Clade B also reside in South America where viruses of Clade A are found. This clade contains eight viruses including four in which are known to be significant human pathogens; Sabia virus, Guanarito, Junin, and Machupo virus. Other viruses of this clade include Tacaribe, Chapare, Cupixi, and Amapari virus. Clade $\mathrm{C}$ is comprised of only two arenaviruses, Latino virus and Oliveros virus; both of which exist in South America and are not known to be pathogenic to humans. Finally, Clade A/Rec is comprised of the virus species that are found in North America and are either pathogenic (Whitewater Arroyo virus) or nonpathogenic (Tamiami virus, and Bear Canyon virus) for humans.

\section{THE ARENAVIRUS LIFE CYCLE}

During viral infection, binding with host receptors has been shown to be the signal that facilitates entry into the host cells [9]. The most common, and first to be discovered, cellular receptor used by OW arenaviruses, and clade $\mathrm{C}$ of the NW group, is alpha-dystroglycan ( $\alpha-D G)[10,11]$. Dystroglycan is a cellular component found in most mammalian tissues, and is used as a linkage between the extracellular matrix (ECM) and the actin cytoskeleton of cells. Alpha-DG 
normally binds a component of the extracellular matrix, laminin, while $\beta-D G$ is the membrane portion of the receptor that anchors the receptor with its binding to dystrophin in the cytoplasm. Dystroglycan undergoes processing of the core protein that results in extracellular component, $\alpha-D G$, and transmembrane protein $\beta$-DG. During processing of DG, $\alpha-D G$ goes through a complex O-glycosylation process [12]. This process is essential for $\alpha-D G$ function as both a cellular component and receptor for arenaviruses $[13,14]$. It has been shown that viral attachment is dependent upon modification of like-acetylglucosaminyltransferase (LARGE) of DG [15], and binding affinity is influenced by the length of these LARGE-derived glycans [13]. A genome-wide study of residents in Western Africa showed that there is a positive selection for specific LARGE alleles in these populations [16-18], indicating that DG modification may play a potential role in viral-host evolution. However, it is still unclear whether or not these selections of LARGE alleles play any role in susceptibility or transmission of these viruses. Rojek and colleagues have hypothesized that $\alpha-D G$ binding by LASV destabilizes the membrane due to its lack of binding to laminin, thus creating a disturbance in the membrane and intracellular signaling, contributing to LF disease pathogenesis [19].

Not all arenaviruses bind $\alpha-D G$ with equal affinity. Differences in receptor binding are also related to infection seen with LCMV; LCMV-WE 54 and LASV strains have a high affinity for $\alpha-D G$, and cause persistent infection. However, those with lower affinity, are readily cleared (e.g. LCMV WE HPI, WE2.2, and ARM) [10, 20-24]. Serine at position 153, and Leucine at position 260, have 
been associated with high-affinity binding of a-DG [24-26]; however, LCMV-WE HPI encodes S153 and L260, but binds a-DG poorly, suggesting that additional residues may play a role in $\alpha-D G$ binding. Hastie et al. identified that $Y 155$ was also required for high-affinity binding to $\alpha-D G$, as well as H136 and R190 [27]. Additionally, this group investigated the role of full-length LCMV GPC or the GP1 subunit. Previous research has reported that LASV required full-length GPC to pull down $\alpha-D G$ and not GP1 alone [28, 29], and indeed, LCMV showed similar results [27].

Along with $\alpha-D G$, there have been reports of a number of alternative receptors that OW arenaviruses may utilize to attach to and enter into host cells. These receptors include cell surface receptor Axl and Tyro3/Dtk, both of which are Tyro3/Axl/Mer (TAM) tyrosine kinases; as well as C-type lectins DC-specific ICAM-3-grabbing nonintegrin (DC-SIGN) and LSECtin [30, 31]. Tyro3 and AxI are broadly expressed in a number of different mammalian tissues and are involved in the removal of apoptotic cells $[32,33]$. Given the co-expression of viral receptors DG and TAM, this supports the idea that LASV and other OW arenaviruses appear to have an incredibly complex receptor usage, which is still not entirely understood.

Once bound to $\alpha-D G, L C M V$ and LASV enters cells via a unique form of endocytosis that is clathrin, caveolin, dynamin, and actin-independent [34-38]. It has recently been discovered that when LASV binds, $\beta$-DG is phosphorylated, which results in dissociation of $\beta-D G$ from the cytoskeleton and may be used in facilitating endocytosis [39]. Additionally, it has been recently been identified 
that LASV and LCMV utilize sodium hydrogen exchangers (NHEs), indicating that viral entry is associated with micropinocytosis giving additional support for a micropinocytosis-like pathway [40]. The path of viral entry through micropinocytosis is not new to virology. A number of other viruses including human papilloma virus (HPV-16), adeno-associated virus 2 (AAV2), and Influenza A virus (IAV) utilize a form of micropinocytosis during viral entry [41]. Other factors associated with LASV entry include the need for GTPase Cdc23 and the downstream effectors PAK1 and N-Wasp, however factors including Rac1, RhoA, the Arp2/3 complex, myosinll, and myosin light chain kinase are not essential during LASV entry [40].

Many factors of LASV entry support the concept that LASV entry uses a unique form of micropinocytosis. Macropinosomes, like those of early endosomes, must go through a maturation process [42]. However, the maturation process is not completely understood for LASV. What is known, is that LASV passes through the late endosome on its path to replication [43], but the point in which LASV moves from a micropinocytosis pathway into the classical late endosome pathway is still unknown. What has been identified is that delivery of the multivesicular body to the late endosome is dependent upon microtubular transport $[35,44]$. The virus-receptor complexes are sorted into intraluminal vesicles with the help of ESCRT (endosomal sorting complexes required for transport) proteins to be transported to the late endosome [43]. This method of trafficking results in a bypassing of the early endosome, the compartment that contains TLRs responsible for recognizing viral RNA. The 
utilization of this trafficking system may provide pathogenic OW arenaviruses the ability to avoid triggering immune responses within host cells [45]. Once LASV has reached the late endosome, acidification causes LASV to switch from $\alpha-D G$ to an intracellular receptor, LAMP1 [28].

Lysosome-associated membrane protein (LAMP) 1 , is a protein that is partially responsible for the maintenance of lysosomal integrity at very low $\mathrm{pH}$ $[46,47]$. Binding of LAMP1 is driven by a triad of histidines on the GP1 and that binding of LAMP1 triggers the LASV spike to catalyze membrane fusion by potentiating its response to $\mathrm{pH}[29,48]$. While this histidine triad is conserved among other OW arenaviruses, LAMP-1 utilization has been shown to be specific for LASV, and LAMP1 is not utilized by LCMV or other OW arenaviruses, regardless of $\alpha-D G$ affinity [27, 49]. Additionally, recent research has identified that LAMP 1 increases LASV efficiency during entry, and increases the $\mathrm{pH}$ of the late endosomes/lysosomes to promote replication [50]. While LAMP1, even in low amounts, supports robust entry of LASV, LAMP1 is not necessary for viral entry, although attenuation is seen without LAMP1 present [50].

While there is not a complete understanding of viral assembly and budding of arenaviruses, the $Z$ protein, or matrix protein, has been shown to be essential for budding progeny, as it is capable of forming virus like particles (VLPs) when expressed alone [51]. During the budding process, $Z$ forms an inner layer under the viral envelope where it is able to recruit NP and GPC to the viral particles, and has been shown to require ESCRT pathway to be successful [52-55]. It does seem that one or more proline rich domains in the C-terminus, 
and/or the YxxL motif in the RING domain need to be present in order for productive assembly to occur, however the number and combinations of these domains vary among OW arenaviruses.

\section{THE ARENAVIRUS GENOME}

Arenaviruses are enveloped viruses, decorated with glycoprotein spikes on the outside, that have a single-stranded, negative-sense, bi-segmented RNA genome. Arenaviral particles can range from 40 to 200 nanometers in diameter $[6,56]$. One segment, the large $(L)$ segment, encodes the small RING finger protein that serves as the matrix protein $(Z)$, and the RNA dependent RNA polymerase [57]. The other segment, the small (S) segment, encodes nucleoproteins (NPs) and glycoproteins (GP) [57]. The viral genome uses an ambisense coding strategy, synthesizing two polypeptides in opposite orientation, which is separated by a noncoding intergenic region (IGR), with a hairpin structure which contains 2 segments (Figure 2 and 3 ). The term ambisense was coined to describe the one region on both the $S$ and $L$ segments that were negative sense, and in a nonoverlapping region, the segments are pseudo-positive sense. Arenaviral RNA acts as the template for transcription and replication to occur.

Due to the nature of the ambisense genome, the NP and $L$ genes are transcribed into genomic complementary mRNA. However, the GP and Z RNA 


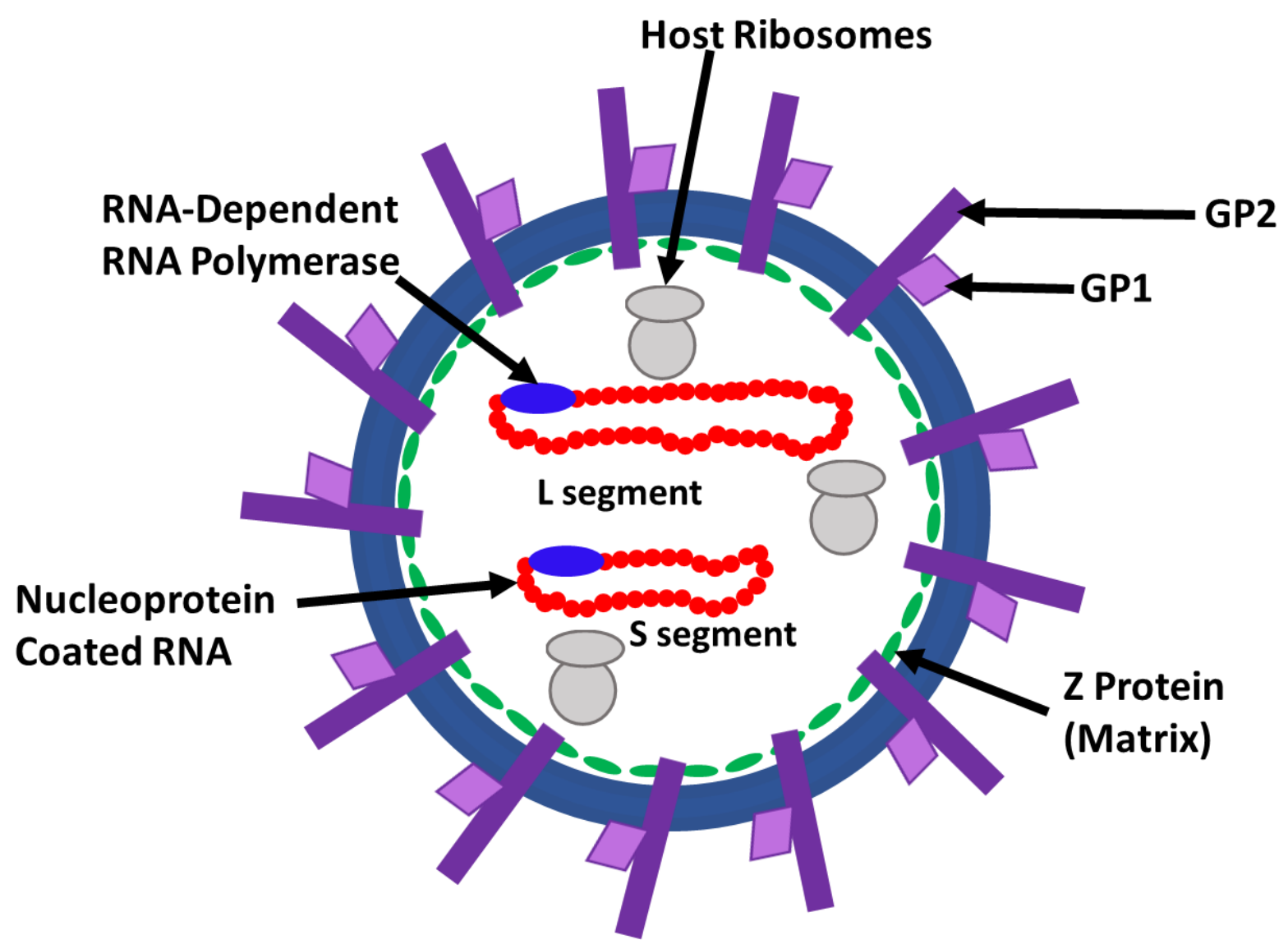

Figure 2: Arenavirus virion structure.

The Glycoprotein (GP) 1, responsible for attachment to cellular receptors, and GP2, responsible for fusion of host membranes, are depicted in dark and light purple, respectively. Beneath the viral envelope (dark blue) the matrix (Z) protein is depicted in green. Inside the virion are host ribosomes, that give arenaviruses their "sandy" appearance in electron micrographs, are depicted in gray. Additionally, ribonucleoprotein complexes are composed of nucleoprotein (NP- in red) that encapsidate viral RNA. The ribonucleoprotein complexes are associated with RNA-dependent RNA polymerase $(L)$ depicted in blue. Image adapted from Wolff et al. 2013 [52]. 

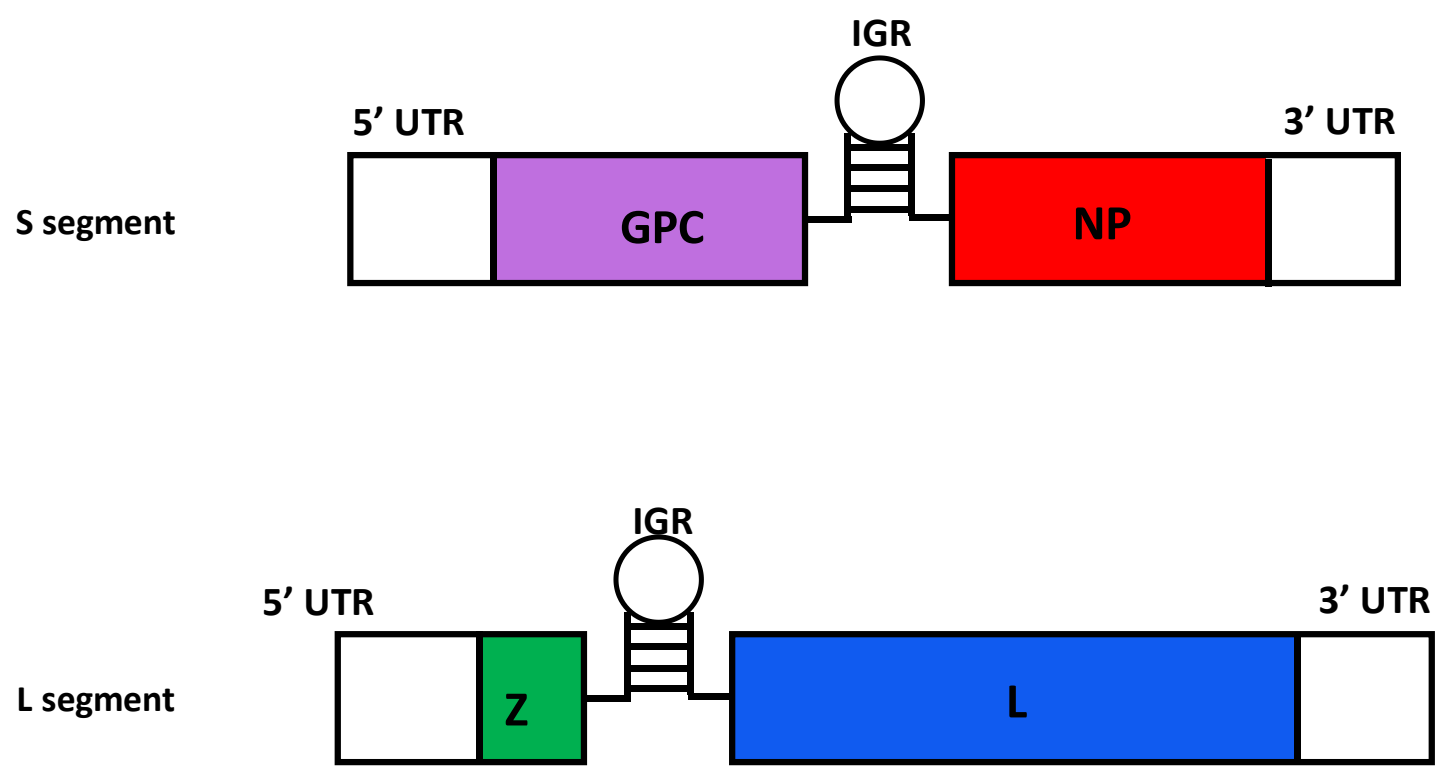

Figure 3. Arenavirus Genome.

The genes of the small (S) segment encode the glycogroptein complex (GPCpurple) and Nucleoprotein (NP-red). The Matrix protein (Z), and RNA-Dependent RNA Polymerase (L-blue) are encoded on the Large (L) segment. These genes on two segments that are arranged in ambisense orientation. Image adapted from Wolff et al. 2013 [52]. 
must go through genomic RNA replication, and only then these genes are transcribed into mRNAs being translated into GPC and Z proteins. Highly conserved regions at the 3 ' end of both the $L$ and $S$ segments, suggests these are the site of viral polymerase initiation $[58,59]$. Through the use of electron microscopy, scientists have observed that both the 5' and 3' ends of the $L$ and $S$ genome segments form panhandle structures thought to have a role in controlling RNA synthesis, and these arenavirus genomes and antigenomes show a high degree of complementarity between their $5^{\prime}$ and 3 ' termini [60-63]

Due to the inconsistency in the location of S-derived NP and GP transcription termination, it has been predicted that the structural motif, rather than the sequence of the IGR, is what promotes the release of viral polymerase from the template RNA $[59,61]$. There are significant differences between the location of S and L IGR sequences in regards to RNA folding, however, among isolates and strains of the same species, S and L IGR sequences are highly conserved. Viruses such as LASV and LCMV contain a single stem loop, whereas MOPV and TACV are predicted to have two $[59,64]$.

The glycoprotein complex (GPC) is translated as a precursor polyprotein, which is translocated into the ER where a stable signal peptide (SSP) is cleaved from its $\mathrm{N}$-terminus. Further posttranslational modifications result in two subunits; the peripheral GP1 responsible for receptor attachment, and the transmembrane protein responsible for membrane fusion, GP2 [58]. The SSP remains associated with the GP1 and GP2 virion and serves several important functions. One function is that the SSP is required for cleavage and maturation 
of the GPC. Additionally, the SSP associates with the transmembrane domain of the GP2, and potentially may play a role in $\mathrm{pH}$-dependent fusion of the GP [27, 65-73]. After arenaviruses enter the cell via a micropinocytosis-like pathway, the GPC is exposed to an acidic environment in the late endosome. This $\mathrm{pH}$ change triggers irreversible conformational changes that the virus utilizes for fusion to host membranes.

Previous work has determined that the GP1 from JUNV, LCMV, and MACV all have a similar core of a six-stranded $\beta$-sheet, and most of the helices on the helix-loop face are maintained between the three viruses [27]. The $\mathrm{N}$ - and C- termini of LCMV, MACV, and JUNV GP1 are oriented towards the GP1-GP2 surface, LASV GP1 termini are oriented in the opposite directions [27]. Additionally, these major helixes of LASV GP1 is nearly perpendicular to the orientation of these helices in LCMV, JUNV, and MACV GP1 [27].

The GP2 subunit contains both heptad repeats and fusion regions. Crystallization of GP2 subunits of arenaviruses show six-helix bundles structures that typically are found in the class I viral glycoproteins [74-76]. Research shows that LASV employs both an N-terminal fusion peptide (termed F1), like that of class I viral glycoproteins, and a fusion loop (termed F2), as seen in Ebola virus [77]. Interestingly, the amino acid sequences of these F1 and F2 are well conserved and nearly identical between the arenavirus family [27]. Additionally, there have been extreme differences between the post-fusion form of LCMV GP2 and the pre-fusion form. In post-fusion conformation, HR1 and HR2 form a single helix with a 'T loop' between them, and three copies of each HR1 and HR2 
form an antiparallel 6-helix bundle. In the pre-fusion GP2, HR1 is broken into 4 different segments, HR1a, HR1b, HR1c, and HR1d. HR1a, c, and d form discrete helices, while b forms an extended loop structure. Additionally, the T loop forms two anti-parallel $\beta$ strands, rather than an $\alpha$-helix observed in postfusion structures [27]. These conformational differences suggest that GP2 conformational rearrangements occur in the T loop and in the heptad repeat regions during entry.

\section{INDIVIDUAL VIRUSES OF MEDICAL CONCERN}

\section{LASSA VIRUS}

Though Arenaviridae is a highly diverse group of viruses, the most significant of the OW group is Lassa virus (LASV). LASV is the most pathogenic of the OW arenaviruses, and is responsible for several hundred thousand infections, and thousands of deaths annually in Western Africa [45, 78-80]. Although most individuals exposed to LASV mount an immune response to defend against disease, LASV causes a significant number of deaths annually. Interestingly, over $45 \%$ of the populations in some villages of endemic regions is seropositive for LASV, but it is not well understood why some of the population succumb to disease and others do not [81]. LASV is carried by its natural host Mastomys natalensis. While transmission to human is not completely understood, it is widely accepted that it likely occurs via ingestion of contaminated food-stuffs, or by the inhalation of infectious particles [45]. The 
rodent reservoir for LASV and other OW arenaviruses has been shown to maintain low viral titers without presence of disease onset [82].

LASV is designated by the Centers for Disease Control and Prevention as a category A agent due to its high lethality and transmissibility via aerosols. Currently, ribavirin is the only treatment available for LASV, but caveats to this antiviral strategy include severe side effects, and a requirement for early administration in order to have positive effects on LASV disease [83]. Currently, there are no clinically approved vaccines for Lassa virus. Among several vaccine candidates, a MOPV/LASV reassortant (clone ML-29) has demonstrated safety and high efficacy in all available animal models including immunocompromised NHPs [84-90]. ML-29 is a reassortant virus between two unrelated arenaviruses created in vitro after co-infection of cells with both LASV and Mopeia virus (MOPV). Specifically, ML-29 is composed of the L segment of MOPV and the $S$ segment of LASV [91].

Diagnosing LASV in the early stages of infection is difficult as LASV disease can often be misdiagnosed for other co-endemic infections such as malaria or influenza [92]. Disease manifestation of LASV can range from nonsymptomatic subclinical illness, to organ failure and even death. A number of signs and symptoms may be observed from LASV-infected patients including fever, malaise, nausea, petechial hemorrhage, vomiting, diarrhea [93, 94]. Interestingly, sensorineural hearing loss has been observed in up to one-third of patients [95]. Infections with fatal outcomes often exhibit encephalopathy, mucosal bleeding, shock, and coma [93, 96, 97]. 
While LASV results in the most infections and deaths of the OW arenaviruses, the underlying pathogenic mechanisms and virulence factors are still poorly understood. One predictor of disease survival is the level of viremia. Patients that have a higher load of LASV often have poor prognosis and show exacerbated signs and symptoms of disease [98]. Viremia higher than $3.5 \log 10$ TCID 50, typically result in death $[21,96]$. Alternatively, patients that display a low level of viremia tend to survive [98]. While LASV can cause hemorrhagic manifestations, the death related to LASV is far from typical hemorrhaging due to tissue damage. LASV replicates to high titers in vital organs including the liver, lung, and spleen, however, any histological damage in these organs is not severe enough to cause death. The most common and consistent infectionassociated lesions are seen on the liver of infected patients, and consist of hepatocellular necrosis, mononuclear phagocytic reaction, and focal hepatocellular cytoplasmic degeneration, with a very small amount of immune infiltration [99]. Nonetheless, even these pathological lesions are insufficient to cause death in these Lassa Fever (LF) patients [99].

LASV, unlike other hemorrhagic fevers, does not cause severe hemorrhaging in infected individuals. In the minority of patients that exhibit hemorrhaging, it is mostly present at mucosal surfaces [45]. Thus the amount of blood loss and tissue damage is not sufficient to result in shock and death observed in lethal cases [99]. When significant bleeding does occur, usually thrombocytopenia and platelet dysfunction are co-observed [100, 101]. 
LASV has a non-lytic life cycle, and has been shown to not induce cell death in infected macrophages, endothelial cells, and monocytes [102]. Vascular endothelium has been shown to be permissive to LASV, resulting in high titer production of virus, and death of these cells was not observed [102].

Experimentally infected NHPs, as well as human patients, have shown signs of vascular distress and permeability, followed by shock and death [99, 103]. Although human and NHPs infected with LASV do not show lesions in vascular tissue, vascular permeability is present during infection. The mechanism leading to vascular permeability has yet to be identified, but is hypothesized to be caused by LASV manipulating normal cellular functions of the endothelial tissue, which results in increased fluid leakiness from these tissues. Immune regulators are likely the culprit of this increased permeability of tissue, as such is observed with hemorrhagic fever viruses Ebola (EBOV) and dengue (DENV) [104]. However, LASV does not share the same cytopathic profile, but rather has low levels of IL8 and interferon (IFN)-inducible protein (IP)-10 [105]. In vitro macrophages and dendritic cells (DCs) are not able to be activated when infected with LASV and pro-inflammatory markers are not released [106, 107], and pro-inflammatory cytokines are not detected in the sera of fatally infected LASV patients [105]. Interestingly, human umbilical vein endothelial cells (HUVECs) exposed to LASV responded with no change in IL-8 production, a feature commonly seen in LASV patients. However, when apathogenic MOPV, a close relative to LASV, infected these cells, IL-8 was produced in significant amounts [102], indicating LASV's ability to downregulate innate immune responses. 


\section{LYMPHOCYTIC CHORIOMENINGITIS VIRUS}

Due to LASV's status as a Category A biothreat, LCMV is the prototypic arenavirus utilized to study many aspects of arenaviral pathogenicity. LCMV was originally discovered in 1933 when it was the first arenavirus to be isolated during an encephalitis epidemic in St. Louis [108]. After its isolation, it soon was associated as the agent that was chronically infecting mouse colonies [109] as well as causing aseptic meningitis in humans [110]. LCMV is an OW mammalian arenavirus that can be found worldwide, partly due to its reservoir, the house mouse. This virus can be transmitted vertically in mouse populations via intrauterine infection. These mice that are infected in utero are unable to mount an immune response to LCMV, which leads to development chronic, asymptomatic, life-long infection, and the ability to shed virus in large quantities through bodily secretions [111].

LCMV's natural reservoir is the house mouse, mus musculus, and LCMV can infect humans who come in contact with rodent secretions. While direct human-to-human transmission has not been observed, LCMV infection may be transmitted through solid-organ transplantation, and from an infected mother to her fetus [8, 112-114]. While the house mouse is the natural reservoir for LCMV, other rodent species are capable of being infected with the virus. For instance, the largest outbreak of LCMV occurred in the US where 181 humans in 12 different states came in contact with hamsters from a single distributor that were infected with LCMV $[115,116]$. LCMV has infected approximately $5 \%$ of the human population in the US [117-120], and higher seropositivity rates in other 
areas of the world, including over $36 \%$ in Croatia and Bratslavia in Slovakia [121, 122]. Additional countries and regions have been assayed for LCMV seropositivity including Nova Scotia [123] and North and South Germany [124]. Additionally, countries including Argentina [125], Spain [126], and Italy [127] have measured seropositive human populations (1\%-2.5\%) and rodent populations $(5.6-12.9 \%)$.

When humans become infected with LCMV, the infections are often asymptomatic or mild, resulting in fever, chills, nausea, vomiting, myalgia, and headache. Typically, 1-3 weeks after infection, it resolves itself without treatment [58]. Due to most LCMV cases being minor or having no symptoms associated with them, the true incidence of LCMV infections is not known, but based off of the seropositivity data available, it is presumably low [128]. However, in a minority of the population, progress into aseptic meningitis or meningoencephalitis, with potential for additional neurological complications including transverse myelitis, Guillain-Barre-type syndrome, hydrocephalus and sensorineural hearing loss [129]. While neurological symptoms are most common, non-neurological symptoms including pancreatitis, orchitis, arthritis, parotitis, and pericarditis, have been reported [130]. While these symptoms have been reported, most adults infected with LCMV fully recover, and fatality is exceptionally rare [131].

LCMV infections in healthy individuals are usually not dangerous; however, in immune compromised individuals, LCMV can produce a disease that closely resembles LF [58]. There have been 17 cases of reported LCMV 
infections in transplant recipients, 14 of which were fatal $[8,113,114,131]$. Infection from transplantation has been associated with multisystem organ failure and hepatitis.

While the mortality rate of LCMV is less than $1 \%$ [131], some LCMV infections can be serious, resulting in spontaneous abortion if pregnant women are infected during the first trimester $[132,133]$. Infection in the second and third trimester has been linked to congenital neurological dysfunction including hydrocephalus, macro- or microcephaly, gyral dysplasia, focal cerebral destruction, loss of vision, and chorioretinitis [130, 132-136]. True prevalence of congenital LCMV infection is unknown, due to the similarity to cytomegalovirus infection and congenital toxoplasmosis, but it is estimated that $35 \%$ of infants die from complications of congenital LCMV.

\section{TRANSMISSION OF RODENT-BORN ARENAVIRUSES TO HUMANS}

Transmission of arenaviruses from their natural rodent reservoirs to human hosts has been identified to occur via routes of ingestion of contaminated food stuff or inhalation of infectious particles [45, 137]. An epidemiological study in the Republic of Guinea showed a link between the consumption of contaminated food as a risk factor for rodent-to-human transmission [137]. There have been some in vitro studies investigating arenavirus interactions in culture models of kidney and bronchial epithelia $[138,139]$. However, there has yet to 
be in vitro investigation using cell types representative of the sight of intragastric infection.

During rodent-to-human transmission, epithelial cells of the gastrointestinal system and upper respiratory tract are among the first host cells to come into contact with the viral pathogen. Transmission via aerosolized viral particles is generally considered to be initiated by the interaction of the virus with the apical side of the epithelia, whereas viruses that are transmitted via scratch or bite from an infected host is interacting directly with circulating blood cells or the basolateral sides of epithelial cells [139-141]. The route exposure can alter infection due to receptor location, resulting in difference entry and adherence to cells. In addition, epithelial polarization has been shown to impact the location of viral receptors [138, 142-144]. Interestingly, infection of different organ tissues has resulted in differences in entry and exit patterns, as seen with infection of thyroid and colon cell lines with Semliki forest and Sindbis viruses [145]. Additionally, members of the same virus family have shown opposing results in replication within the same tissue [146], making transmission of viruses even more unclear.

\section{IMMUNE RESPONSE TO OW ARENAVIRAL}

When humans do become infected with LASV, a T-cell mediated response to LASV seems to be critical in survival [147-150]. In NHP studies, animals that survived LASV infection showed T-cell activation to be one key to survival, as 
monkeys that showed a delay in T-cell activation (as well as a high viral load), did not survive [151]. Clinical observations indicated that the antibody response does not seem to be effective in controlling LASV infection, indicating that antibodies produced against LASV, are non-functional and not neutralizing antibodies. This inability to control viral infection may be a result of NK cells or downregulation of cytotoxic T-cell populations, both of which have yet to be investigated in patients with LF. The levels of antibodies in sera did not correlate with recovery or progression, signifying that these antibodies are not functional or capable of neutralizing viral activity [98].

Macrophages and antigen presenting cells (APCs) are the first cells to come in contact with the virus after the virus crosses the epithelial barrier. Notably, in an in vitro study, LASV was seen to readily infect human dendritic cells (DCs) and macrophages, but was unable to activate these cells [106]. This was signified by lack of increase in several immune mediators including tumor necrosis factor alpha (TNF $\alpha$ ) and interleukins such as IL-1 $\beta$, IL-6, or IL-19, as well as the absence of costimulatory molecules such as CD40, CD54, CD80, CD86, or HLAs; all of which signify a lack of DC phagocytic activity [106]. A recent study however, has compared levels of pro- and anti-inflammatory cytokines and chemokines from the serum of LASV-infected patients [105]. These samples included serum from LASV-infected humans fatally-infected and non-fatally-infected, as well as uninfected control samples. Interestingly, proinflammatory markers IL-8 and interferon-inducible IP10 protein, were significantly higher in patients with non-fatal LASV infections than in control 
samples. However, patients with fatal LASV infections, had little or undetectable levels of IL-8 and IP10. Also notable, that although TNFa concentrations were not elevated in any of the LASV-infected patients, those patients did show an increase in TNFa receptors compared to uninfected individuals. Additionally, IL-6 has been noted as an important factor for determining the outcome of patients infected with LASV [98]. IL-6 has been seen to be abundant in fatally infected NHPs as well as humans, and along with elevated liver enzymes, aspartate aminotransferase (AST) and alanine aminotransferase (ALT); all potential biomarkers for progressed LASV infection [90, 98, 151-156].

Mopeia virus (MOPV) is genetically a close relative to LASV; however, MOPV lacks pathogenic potential in humans and non-human primates, and induced protective immune responses against LASV challenge in NHPs [149]. MOPV, like LASV, lacks the ability to activate DCs in vitro, however, MOPV is able to activate macrophages, as signified by increases in the transcription of several immunoregulatory genes for interferon alpha (INF $\alpha$ ), IFN $\beta$, TNF and IL-6 [157].

An effective innate response to viral infection, that results in an adaptive response, is essential for the control and prevention of disease manifestation. Additionally, strong activation of these responses is typical of non-pathogenic arenaviruses [102, 158-162]. However, LASV is known for its suppression of immune systems in infected patients. One mechanism in which OW arenaviruses do this is by preventing type I IFN production. Several studies have shown that LASV NP may be responsible for inhibiting type I IFNs, related to the 
establishment or maintenance of persistent infections in natural rodent reservoirs. These NPs result in silencing of innate and adaptive-immune responses by degrading double-stranded RNAs (dsRNAs) [163, 164]. dsRNAs act as pathogen-associated molecular patterns (PAMPs) and have been shown to trigger several different proteins such as RIG-I and MDA5, both of which trigger an antiviral signaling cascade $[165,166]$. Notably, NP of tacaribe virus (TCRV), a virus isolated from ticks with no identified mammalian host, does not inhibit IFN type I response [54].

Additionally, LCMV-WE and LASV have been shown to down-regulate innate pro-inflammatory responses in vitro, and in vivo, compared to nonpathogenic LCMV-ARM and MOPV $[90,102,153,156,167]$. LCMV-WE and LASV-infected cells inhibited Toll-like receptor 2 (TLR2)/MyD88 adaptor-like (Mal)-dependent cytokines. In contrast, LCMV-ARM and MOPV induced NF-kBmediated proinflammatory responses in monocytes, macrophages, and in epithelial cells [167]. Interestingly, previous research has shown that internalization and viral replication is required for the TLR2/Mal-dependent signaling. When cells were exposed to UV- or heat-inactivated LCMV-ARM, the cells did not induce TLR2/Mal-dependent pro-inflammatory cytokines [167], indicating that successful viral infection needs to occur, and not just interaction with viral receptors and the host. 


\section{ANIMAL MODELS MIMICKING NATURAL TRANSMISSION}

Natural transmission from rodents to human hosts occurs through ingestion or inhalation of contaminated food stuffs or aerosolized infectious particles. Studies in mice and NHPs, using inhalation and ingestion routes of infection provide insights to arenaviral transmission $[155,168,169]$. Mice that were treated intravenously (i.v.) had viral dissemination of LCMV within 72 hours post infection. When infected intragastrically (i.g.), mice showed a delay of dissemination, but by 96 hours post infection, all tissues that showed positive infection via i.v. were positive in the i.g. infected mice as well [170]. NHPs infected either via i.g. or i.v. with LCMV-Armstrong (ARM) or LCMV-WE had differential infections. LCMV-ARM produces a sub-clinical infection when administered to NHPs intravenously, as compared to LCMV-WE in which animals succumb to fatal infection with LF-like hepatitis and hemorrhage manifestations. Interestingly, when infected i.g. with a lethal dose of LCMV-WE, some monkeys recovered from manifested infection [171]. Interestingly, when these NHPs were challenged with a lethal dose of LCMV-WE after being intragastrically infected with LCMV-ARM or -WE, these animals did not succumb to LF-like disease as seen with PBS pre-treated animals [171]. Therefore, intragastric infection of NHPs with LCMV resulted in limited viral dissemination, that led to protective immunity. While animal models have been deployed to further characterize arenavirus pathogenicity several questions remain, and there is a need for an in 
vitro model for studying interaction of mammalian arenaviruses with epithelial cells derived from major gates of virus entry.

\section{POLARIZED EPITHELIAL MODELS}

While there have been in vivo studies mimicking natural routes of infection, an in vitro system has yet to be developed to investigate the interaction of virus with intestinal epithelia at the site of intragastric inoculation. Caco-2 cells have been an established model of intestinal epithelial cells for some time. The cells were isolated from a gastrointestinal tumor by Jorgen Fogh in the 1970s [172]. Although many cell types had been isolated from tumors, these cells were different due to their ability to spontaneously differentiate upon reaching confluence. Upon further investigation in these cells, Caco-2 cells were found to mimic, quite closely, enterocytes of humans. These cells started to polarize once confluency was reached, and developed characteristics that other cell lines did not provide. Caco-2 cells are commonly grown on permeable filter inserts. These inserts allow the cells to obtain morphological and function characteristics of enterocytes [173]. Over time, Caco-2 cells change in dimension, growing taller and width of these cells decreased, moving to a more enterocyte-like cell [173]. Additionally, via electron microscopy, after three days occluding junctional complexes were formed; and by day 6 , desmosomes were formed and the lateral membranes of neighboring cells were interlocking. Additionally, the formation of the brush border and microvilli were becoming more organized and numerous. 
By day 16, these Caco-2 cells had formed a monolayer with a morphology similar to that of simple columnar epithelium of the small intestine. Additionally, an increase in the amount and location of alkaline phosphatase, sucrase, and aminopeptidase was observed as the monolayers polarized [173, 174].

In addition to morphological studies, functional studies have also been investigated, such as transepithelial electrical resistance (TEER) being measured. TEER is a way to test the integrity of the Caco-2 monolayers by measuring the resistance across the membranes. TEER measurements increased over time as these cells polarized on inserts, suggesting a confluent monolayer had been formed [173]. Furthermore, transport studies can also be done to test the permeability of this monolayer. Several studies have investigated the permeability coefficients and absorption data in humans compared to Caco-2 cell lines and found high correlation between the two [175178]. Not surprisingly, the transport of molecules slowed as time of polarization of these cells increased, signifying that monolayer formation and integrity increased over time.

While Caco-2 cell characteristics closely mimicked that of enterocytes, these cells did possess some characteristics of colonocytes [179]. Interestingly, while these cells were found as a tumor in the colon, it has yet to be explored as to why their morphology and physiology tend to be more like enterocytes than colonocytes. 


\section{OBJECTIVE OF DISSERTATION}

LASV is the most pathogenic of the OW arenaviruses infecting thousands of people annually through ingestion of contaminated food stuffs or inhalation of infectious particles. However, the role of the intestinal epithelial cells at the site of intragastric infection has yet to be explored. Furthermore, factors determining differences in viral pathogenesis between close genetic relatedness has yet to be determined. Collectively, the current studies show support that viral dissemination and interaction with epithelia may be host, tissue, and viral specific. We explored these topics with the following aims:

1) To investigate the interaction of LCMV-ARM, LCMV-WE, Mopeia virus (MOPV), and a reassortant virus simulating LASV, ML-29, with polarized intestinal epithelial cells mimick the role of barrier systems in arenaviral infection.

2) To characterize differences between pathogenic and non-pathogenic strains of LCMV, Armstrong and WE respectively, during intracellular trafficking in epithelial and macrophage cell lines. 


\section{CHAPTER 2:}

THE INTERACTION OF MAMMALIAN ARENAVIRUSES WITH POLARIZED

\section{EPITHELIAL CELLS}

Warner NL, Jokinen JD, Beier JI, Sokoloski KJ, Lukashevich IS. Mammarenaviral Infection Is Dependent on Directional Exposure to and Release from Polarized Intestinal Epithelia. Viruses. 2018;10(2). doi: 10.3390/v10020075. PubMed PMID: 29439402. 


\section{OVERVIEW}

Mammarenaviruses are single-stranded RNA viruses with a bisegmented ambisense genome. Ingestion has been shown as a natural route of transmission for both Lassa virus (LASV) and Lymphocytic choriomeningitis virus (LCMV).

Due to the mechanism of transmission, epithelial tissues are among the first host cells to come in contact with the viruses, and as such they potentially play a role in spread of virus to naïve hosts. The role of the intestinal epithelia during arenavirus infection remains to be uncharacterized. We have utilized a wellestablished cell culture model, Caco-2, to investigate the role of intestinal epithelia during intragastric infection. We found that LCMV-Armstrong, LCMVWE, and Mopeia (MOPV) release infectious progeny via similar patterns. However, the reassortant virus, ML-29, containing the L segment of MOPV and $S$ segment of LASV, exhibits a unique pattern of viral release relative to LCMV and MOPV. Furthermore, we have determined attachment efficacy to Caco-2 cells is potentially responsible for observed replication kinetics of these viruses in a polarized Caco-2 cell model. Collectively, our data shows that viral dissemination and interaction with intestinal epithelia may be host, tissue, and viral specific.

\section{INTRODUCTION}

Arenaviruses are enveloped viruses that have a single-stranded, bisegmented, ambisense RNA genome. The Large $(L)$ segment, encodes the matrix protein (Z), and the RNA dependent RNA polymerase [57]. The Small (S) segment, encodes the nucleoproteins (NPs) and glycoproteins (GP) [57]. 
Initially, arenaviruses were thought to only infect mammals; however, recently arenaviruses have been identified as the causative agent of inclusion body disease in the boid family of snakes [2]. Hence, Arenaviruses have been separated into two genera on the basis of their natural reservoir hosts; mammarenavirus, which infect mammalian hosts, and reptarenavirus which infect reptilian host species [3]. Among the mammarenavirus genera, there is further subdivision into the Old World (OW) LCMV-Lassa virus complex and New World (NW), Tacaribe virus complex [3, 4].

Lassa virus (LASV), the causative agent of Lassa fever (LF), is recognized as the most prevalent and most pathogenic of the OW arenaviruses. Annually in Western Africa, there are several hundred thousand clinical LASV infections, and thousands of deaths due to LF $[45,78-80]$. Although LASV causes a significant number of deaths, the majority of infections are apparently subclinical, or not severe enough to warrant emergency medical intervention, as over $45 \%$ of the population in endemic regions is seropositive for LASV; however, why some of the population develop disease and others do not it is not well understood [81]. Most recently, a study of almost 200 LASV sequences has shown that reservoirto-human transmission is a primary driving force of LASV epidemics in Western Africa [17]. LASV is carried by its natural host Mastomys natalensis, and it is widely accepted that transmission of LASV to humans likely occurs via the ingestion of contaminated food-stuffs, or by the inhalation of infectious particles [45]. Indeed, an epidemiological study in the Republic of Guinea showed a link 
between the consumption of contaminated food as a risk for rodent-to-human transmission [137].

Due to its high lethality and transmissibility via aerosols, LASV is categorized by the Center for Disease Control and Prevention as a category A select agent. Currently, there are no clinically approved vaccines for LASV; and the antiviral drug ribavirin is the only treatment available for LASV infection. Nonetheless, caveats to this antiviral strategy/treatment regimen include severe side effects, and the requirement for early administration in order to have positive therapeutic effects [83]. Among limited vaccine candidates, only a LASV/Mopeia (MOPV) reassortant virus, ML-29, has been demonstrated to induce protective immunity against LASV strains from clade IV (Sierra-Leone, Liberia, Republic of Guinea) and clade II (Nigeria) [84-90]. Specifically, ML-29 is composed of the MOPV L segment, a non-pathogenic relative of LASV, and the S segment of LASV [91]. MOPV and ML-29 share the L RNA encoding L protein (RNA polymerase), and $Z$ protein (matrix). Previous studies have determined that the $L$ RNA segment of MOPV is the major factor of ML29 attenuation in vivo. Comparison of the ML-29 L segment with the parental MOPV L segment revealed the presence of numerous point mutations that may contribute to the attenuated phenotype associated with ML-29 [85]. While this reassortant has ML29 specific mutations in the NP and GP2 proteins encoding by LASV S RNA, the attachment glycoprotein, GP1, is genetically identical to LASV.

Similar to LASV, the prototypic arenavirus Lymphocytic Choriomeningitis virus (LCMV), is genetically and biologically diverse. Transmission of this virus 
has been shown to share a similar mechanism to LASV, with transmission from rodents-to-non-human primates (NHP) and humans. Like the epidemiological study from the Republic of Guinea, a natural route of infection was observed within zoo kept tamarin populations, as animals that consumed LCMV-infected mice succumbed to LF-like illness and disease [180, 181]. Importantly, these results have been recapitulated experimentally via the intragastric inoculation of NHPs with LCMV $[155,182]$.

As mentioned earlier, LCMV strains are genetically and biologically diverse. LCMV-Armstrong (LCMV-ARM), a neurotropic strain, is highly adapted for infection in murine models. As such, exposure of NHPs to LCMV-ARM through either intravenously (i.v.) or intragastrically (i.g.) routes produced deeply attenuated sub-clinical infection $[155,182]$. In contrast, LCMV-WE has limited passage history in mice and tissue culture models of infection, and induced fatal LF-like disease in i.g. and i.v. infected NHPs, providing a surrogate model of LF at biosafety level (BSL)-3 containment [155, 182]. Notably, infections via mucosal (i.g.) inoculation were attenuated during interaction with, and/or crossing the mucosal barrier of the gastrointestinal tract. Therefore, the intestinal epithelial are likely one of the natural gates of rodent-to-human transmission. Interestingly, when the LCMV-ARM or -WE i.g. infected NHPs were challenged with lethal doses of LCMV-WE intravenously, the animals did not succumb to LF-like disease as observed with PBS pretreated animals [182].

Due to the mechanism of transmission of arenaviruses from rodents to humans, epithelial tissue are among the first host cells to come in contact with 
the viruses, and as such they potentially play a decisive role in the spread of virus to naïve hosts [183]. The role of epithelial barriers on infection has been investigated extensively with a number of other viruses; however, the specific role of the intestinal epithelia on arenavirus infection remains to be exhaustively characterized [138, 142-144]. Natural transmission via the intragastric route is generally considered to initiate with the interaction and infection of the epithelial cells from the apical side, whereas basolateral exposure of viruses requires damage, layer such as from a scratch or bite from an infected host, to the epithelial cell layer [139-141]. Here, we investigated the interaction of LCMVArm, LCMV-WE, Mopeia virus (MOPV), and the LASV/MOPV reassortant ML-29 with polarized Caco-2 intestinal epithelial cells, to investigate the role of barrier systems in viral dissemination, and to further elucidate the interactions of $\mathrm{OW}$ mammarenaviruses with the gastrointestinal epithelial. Collectively, our current studies support the model that viral dissemination and interaction with epithelia may be host, tissue, and viral specific.

\section{METHODS}

Viruses and Titration Assays. VeroE6 (C1008) cells, Caco-2 (HTB-37) cells, purchased from American Type Culture Collection (ATCC), 16HBE140 cells (a gift from Jonsson Lab, The University of Tennessee Health Science Center), and Madin-Darby Canine Kidney (MDCK- a gift from Chung Lab, University of Louisville) were grown in minimal essential media using Dulbecco's modified eagle medium (Invitrogen, Carlsbad, CA, USA) containing 10\% fetal bovine serum (Fisher Scientific, Hampton, NH, USA) and 1\% antibiotic-antimycotic (Life 
Technologies, Carlsbad, CA, USA) in a humidified chamber at $37{ }^{\circ} \mathrm{C}$ under $5 \%$ CO2. HBE cells were seeded on plates and flasks coated in a collagen, fibronectin solution containing the following concentrations in a $100 \mathrm{~mL}$ solution: $88 \mathrm{~mL}$ of LHC basal medium (Invitrogen), $10 \mathrm{~mL}$ bovine serum albumin (1 mgBSA/mL LHC media), $1 \mathrm{~mL}$ of Vitrogen 100 (BD Biosciences), $1 \mathrm{~mL}$ Human Fibronectin (BD Laboratories).

Cells were infected with LCMV-Armstrong (strain 53b), LCMV-WE (strain 54), Mopeia virus (MOPV, strain AN20410), or the Mopeia/Lassa reassortant virus, clone ML-29 [184, 185]. All viral stocks were generated using low multiplicity of infection (MOI) and stocks with titers ranging from $1 \times 107 \mathrm{PFU} / \mathrm{mL}$ to $1 \times 108 \mathrm{PFU} / \mathrm{mL}$ were stored at $-80^{\circ} \mathrm{C}$ until needed [88].

Viral titers were determined using a standard plaque assay with minor modifications [167]. Briefly, VeroE6 cells were seeded in the wells of a 12-well cell culture plate, and incubated until $80-90 \%$ confluent. Virus samples were serially diluted, and used to infect the Vero cells. Infection was carried out for $1 \mathrm{~h}$ in $37^{\circ} \mathrm{C}$. After this period, the cells were washed with DMEM without phenol red, and a semi-solid overlay containing $1 \mathrm{X}$ MEM, $5 \%$ FBS, and $0.5 \%$ Avicel (FMC BioPolymer, Philadelphia, PA, USA) (LCMV and ML-29) or 0.5\% Agarose (MOPV). Cells were then incubated in a $37^{\circ} \mathrm{C}$ humidified chamber with $5 \% \mathrm{CO} 2$ for 5 days. The plaque assay cells infected with LCMV and ML-29 had the overlay media removed, were fixed with $4 \%$ paraformaldehyde solution for 15 min and cells were stained with $1 \%$ Crystal Violet solution to identify virusinfected cell foci. For titration of MOPV, virus-infected cells were covered with 
semisolid overlay of $0.5 \%$ agarose $/ 5 \%$ FBS overlay. A $0.04 \%$ neutral red, $0.5 \%$ agarose, $5 \%$ FBS solution was added to wells after 4 days incubation. Both plaque assays have a limit of detection of approximately $80 \mathrm{PFU} / \mathrm{mL}$.

Polarization of Caco-2 Cells and Infection of Polarized Cells. Caco-2 cells were seeded on 24-well plate Transwell inserts (Corning, New York, NY, USA) with a 0.45 micron filter as previously described $[173,186]$. Briefly, 160,000 cells in $0.5 \mathrm{~mL}$ were plated on "apical" side of insert, and $1 \mathrm{~mL}$ of nutrient media was added to each well. For basolateral seeding of the Caco-2 cells, the Transwell inserts were flipped upside down in a sterile container, and $0.5 \times 106$ cells in a volume of $100 \mu \mathrm{L}$ was placed on each Transwell surface. The cells were incubated for $1 \mathrm{~h}$ at $37^{\circ} \mathrm{C}$ to allow for adherence to the membrane. Cells were then put back into the 24 -well plate and $0.5 \mathrm{~mL}$ of nutrient media was added to each insert. Cells were maintained at $37^{\circ} \mathrm{C}$ in $5 \% \mathrm{CO} 2$ for 21 days until polarization was completed. Media was changed every $2-3$ days with fresh media for the entirety of the polarization process. To determine if polarization of epithelial monolayers was complete, Transepithelial Electrical Resistance (TEER) was measured with an EVOM2 Epithelial Voltohmmeter (World Precision Instruments, Sarasota, FL, USA) as previously described [187]. In experiments with non-polarized Caco-2, cells confluent monolayers on day 3 after seeding were used. Tight junction proteins were analyzed with western blot (WB) and qRT-PCR. Antibodies for WB were obtained from the following: ZO-1 (61-7300), Claudin-1 (Thermo Scientific 51-9000, Waltham, MA, USA), Occludin (Thermo 
Scientific 71-1500). Pre-made primers/probe sets against human ZO-1 (Hs01551861) and Occludin (Hs00170162) were purchased from ThermoFisher.

Polarized Caco-2 cells were infected either apically, or basolaterally, with an MOI of $0.3 \mathrm{PFU} / c e$ ll of the corresponding viruses: LCMV-Arm, LCMV-WE, MOPV, or ML-29. Wells and inserts were washed two times with DMEM without phenol red. To each insert, virus was added, and $1 \mathrm{~mL}$ of DMEM without phenol red was to each well. Infection was carried out for $1 \mathrm{~h}$ at $37^{\circ} \mathrm{C}$ in $5 \% \mathrm{CO}$. After this period, the inserts and wells were washed two times with $1 \times$ DPBS (Invitrogen), and $1 \mathrm{~mL}$ of nutrient media was added to each well and $0.5 \mathrm{~mL}$ was added to each insert chamber. Cells were maintained at $37^{\circ} \mathrm{C}$ in $5 \% \mathrm{CO}$. TEER was measured daily for 5 days, and the tissue culture supernatants from the Transwell inserts and wells were harvested daily and the media was replaced.

Confocal Microscopy. Caco-2 cells were grown apically on 12-well, $0.45 \mu \mathrm{M}$ Transwell inserts (Corning) for 21 Days until a polarized monolayer was formed. Cells were then fixed with ice-cold methanol for $10 \mathrm{~min}$ at $-20^{\circ} \mathrm{C}$, washed and stained on both the apical and basolateral sides of the inserts with antibodies against ZO-3 using monoclonal antibody against zonal occludin-3 (Cell Signaling, cat. \# 3704, Danvers, MA, USA) at a 1:1600 dilution. Alpha-dystroglycan ( $\alpha-D G)$ antibody clone $11 \mathrm{H} 6 \mathrm{C} 4$, recognizing fully glycosylated $\alpha-\mathrm{DG}$, was used at a 1:100 dilution (Milipore, cat. \# 05-593, Billerica, MA, USA). Hoescht 33342 (Thermo Fisher Scientific) was used for nucleus staining at 1:10,000 dilution was added for $10 \mathrm{~min}$. Transwell filters were cut out and placed on microscope slides, 
followed by 10 uL of ProLong Gold (Life Technologies), and a cover slip placed on top. Slides were analyzed using an Olympus FV1000 laser scanning confocal microscope and analysis was done using IMARIS software (Bitplane, Version 7.7.1, Zurich, Switzerland).

Attachment Assay. Caco-2 cells were seeded and polarized for 21 days. Cells were infected with an $\mathrm{MOI}$ of $0.3 \mathrm{PFU} / \mathrm{cell}$ on either the apical, or basolateral, surface of polarized cells for all viruses. The cells were infected at $4{ }^{\circ} \mathrm{C}$ for $1 \mathrm{~h}$ to allow cells to attach to the cell surface, but not penetrate the cell. After the attachment period, the Input samples consisting of the cells and inoculum were directly harvested, and the experimental cells were washed several times with 1XPBS to remove unbound virus particles. Trizol-LS (Invitrogen) was used to harvest all of the aforementioned cells. RNA was isolated according to the manufacturer's directions. Quantitative Real-Time Polymerase Chain Reaction (qRT-PCR) was used to quantitate attached viral particle. Primers and probe for ML-29/MOPV targeting the L segment: Forward (5' TCCTCAATTAGGCGTGTGAA), Reverse (5' TACACATCCTTGGGTCCTGA) and probe (5' CCCTGTTCCCTCCAACTTGTTCTTTG). Primer and probe targeting LCMV-Armstrong L segment: Forward (5' CCT TAA AGA GGT GAG AGC ATG A), reverse (5' TTTCATTGATATTCTTGGTTAGGTG) and probe ( $5^{\prime}$ CAGCCACACCTGGATTCTGTAATTGG). Primer and Probe targeting LCMV-WE L segment: Forward (5' CCT GGA CTC TGT AAT TGG CA), Reverse (5' TTA 
CAT GCT CAG CAG CAC AG), and probe (5' TCA CAG TGG ATT TCA CAC ACA ACC AGA).

The attachment of viral particles was assessed quantitatively via the $\Delta \Delta \mathrm{Ct}$ method [60]. Briefly, Ct values corresponding to the viral targets were normalized internally via the subtraction of the $18 \mathrm{~S}$ rRNA levels detected within each sample. The resulting $\Delta \mathrm{Ct}$ values of the washed tissue culture cells were then compared relative to the bound unwashed Input controls. The resulting $\Delta \Delta \mathrm{Ct}$ values were used to determine the relative quantities of viral nucleic acids in the Bound (washed) and Input (unwashed) samples; these values were then plotted, and attachment was reported as further calculated via the ratio of Basolateral/Apical attachment.

Statistical Analyses. Statistical significance was analyzed using 3 biological replicates per experimental time point, using a Standard Student t-Test. All statistical values of $p \leq 0.05$ were deemed as statistically significant.

\section{RESULTS}

Identification of appropriate models to study the interaction of mammalian arenaviruses with epithelial tissue. Mammalian arenaviruses have been shown to be transmitted to human hosts via inhalation and ingestion of contaminated particles $[45,137]$. However, cell culture models have not been well established to investigate the exact nature of interaction and cellular mechanisms during arenaviral infection, as well as a model to investigate potential differences in pathogenicity between these viruses. There have been 
several studies investigating in vitro models mimicking the bronchial tissue in mice [139] as well as a model investigating excretion of these viruses by utilizing Madin-Darby canine kidney (MDCK) epithelial cells [138]. To investigate potential differences in host-viral interactions between different arenaviruses and strains of different pathogenic potential, we sought to explore different cell culture models to identify and classify viral-host patterns with cell lines that were capable of polarizing, in order to mimic interactions in vivo. Our goal was to identify one or more in vitro models suitable of characterizing differences between our viruses of interest.

Inhalation of viral particles has been shown to be one way in which humans may become infected with mammalian arenaviruses. In addition to natural infection, multiple studies have been conducted to investigate this route of infection both in vivo and in vitro [139, 188, 189]. Here, our goal was to utilize a human bronchial epithelial (HBE) cell line that closely mimicked an in vivo model. In order to determine whether these cells would be a beneficial model to use, viral growth kinetics were assessed by standard plaque assay in both polarized and non-polarized HBE cells (Fig. 4). LCMV-Arm showed poor replication in polarized and non-polarized HBE cells, reaching only 1-1.5 logs increased replication by 5 days post infection. The poor replication seen in both polarized and non-polarized HBE cells indicates that they are a poor model to utilize in determining the role of epithelial barriers during arenaviral infection and studies were not further pursued. 


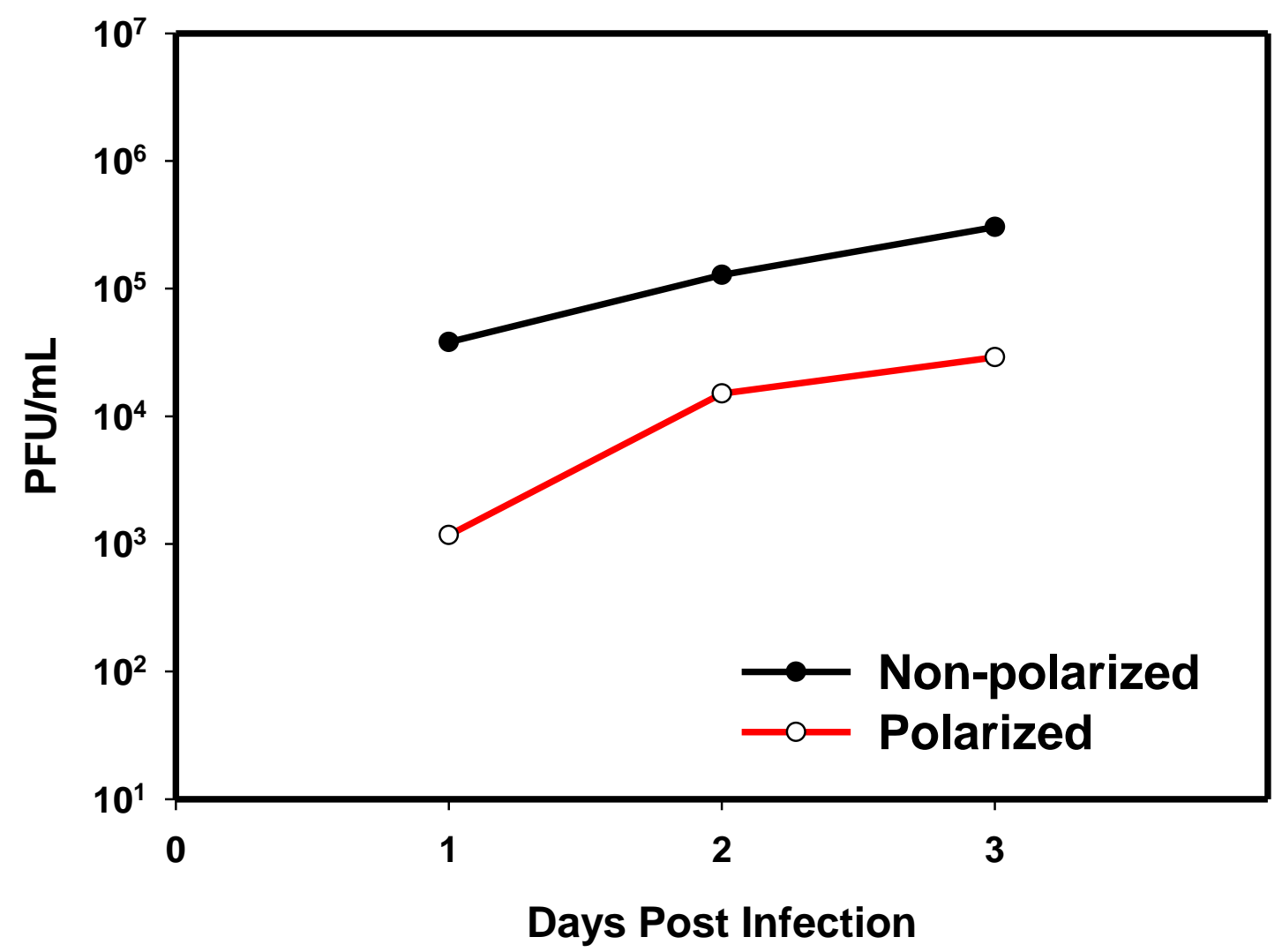

Figure 4 LCMV-Armstrong does not replicate well in polarized human bronchial epithelial cells

Human bronchial epithelial cells were grown on 12-well tissue culture plates and infected with LCMV-ARM at an MOI of 0.3PFU/cell. Supernatants were collected every 24 hours for 3 days. Supernatants were tested using a standard plaque assay. 
Due to arenaviral transmission being involved in aerosolized viral particles, most likely due to the excreta of infected rodents hosts, the next approach was to investigate a cell line that mimicked the excretion of viral particles from the host. Previous studies have utilized the well-established Madin-Darby Canine Kidney (MDCK) epithelial cell line to investigate cellular factors associated with LASV entry and release [138]. Here, we investigated viral replication to determine if MDCK cells were a suitable model to determine viral-host interactions at the epithelial layer, as well as to determine if polarization of these cells played any role on replication (Fig. 5). LCMV-ARM (Fig. 5 A) and LCMV-WE (Fig. 2 B) both replicated well in both polarized and non-polarized MDCK cells. LCMV-ARM reached peak titers of around $1 \times 10^{7}$ in non-polarized epithelia, and $1 \times 10^{6} \mathrm{PFU} / \mathrm{mL}$ in polarized MDCK cells (Fig. $5 \mathrm{~A}$ ).

Interestingly, LCMV-Arm replicated about 2 logs lower in polarized MDCK cells as compared to non-polarized MDCK cells. However, LCMV-WE replicated to similar titers, $1 \times 10^{7}$, in both polarized and non-polarized cells, showing no significant difference based on polarity of these cells (Fig. 5 B). Due to LCMV being a close relative, but not entirely indicative of LASV behavior, ML-29 was utilized to determine how LASV-GP interacted with MDCK cells (Fig. 5 C). Interestingly, ML-29 had very poor replication kinetics in both polarized and nonpolarized MDCK cells, reaching only peak titers of about $5 \times 10^{\wedge} 4$ in non-polarized cells and $5 \times 10^{3}$ in polarized epithelia.

Due to the importance of mimicking LASV infection, we sought to identify an epithelial cell line that resulted in successful replication of both LCMV and 

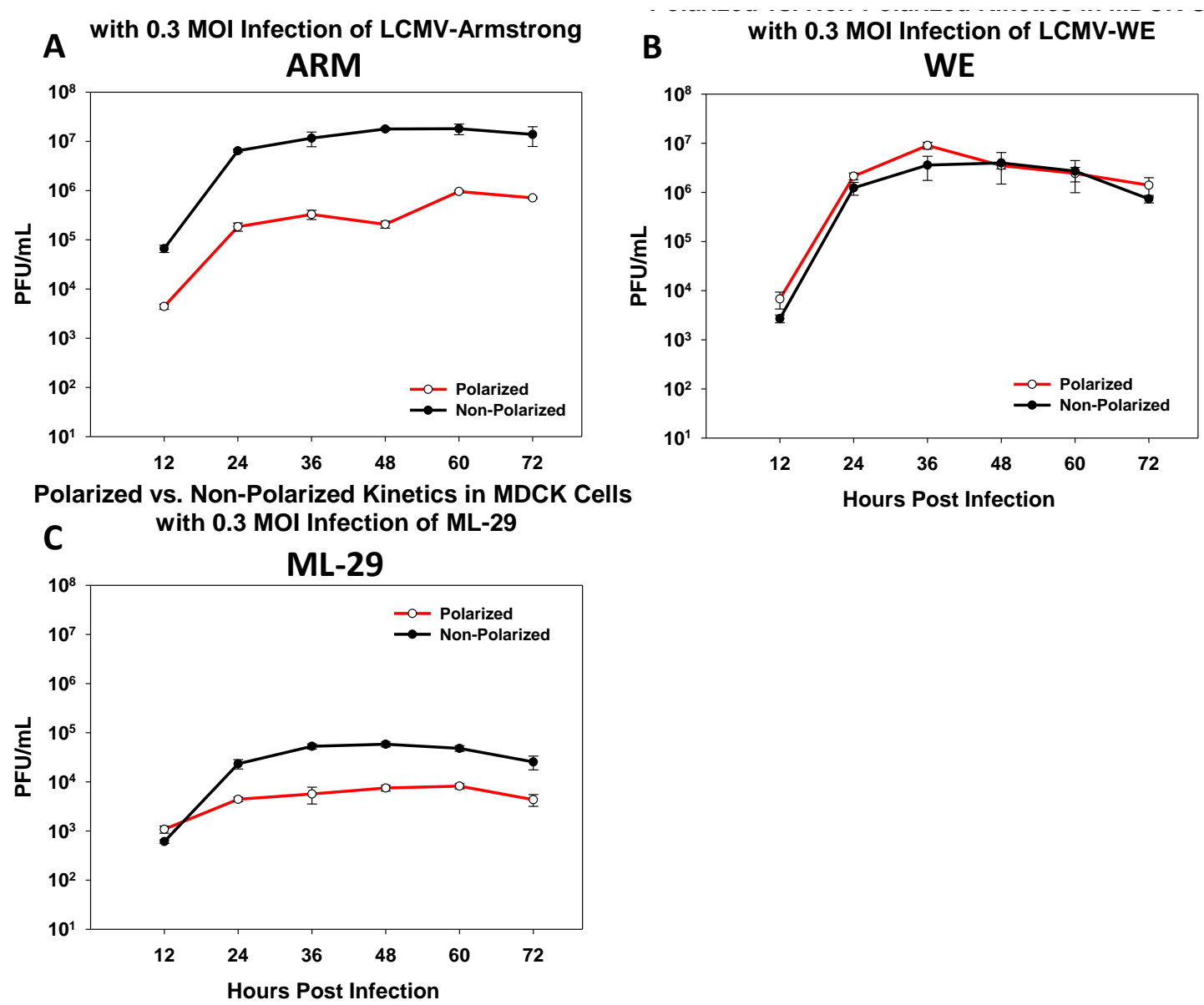

Figure 5. LCMV-ARM, but not WE is impacted by polarization of MDCK cells MDCK cells were non-polarized (black) or polarized (red) in 96-well plates. Cells were infected at an MOI of 0.3 PFU/cell with LCMV-ARM (A), LCMV-WE (B), or ML-29 (C). Supernatants were collected every 12 hours for 72 hours and tested using a standard plaque assay. Values shown are the means of 3 biological replicates with the error bar representing the standard deviation of the mean. 


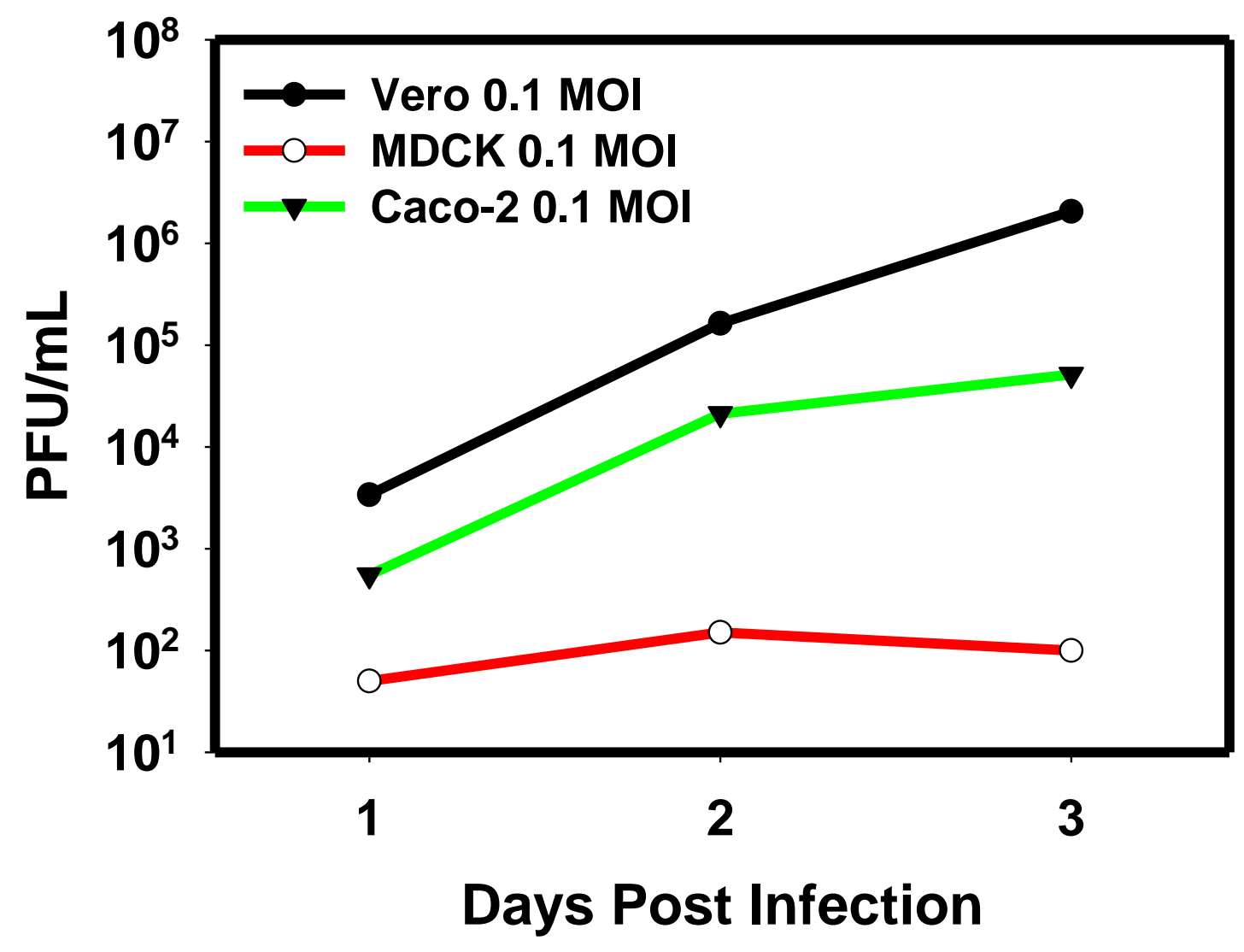

Figure 6. MDCK cells are not permissive to LCMV-Armstrong MDCK cells, VeroE6 cells, and Caco-2 cells were infected with an MOI of 0.1 $\mathrm{PFU} / \mathrm{mL}$ of LCMV-Armstrong. Supernatants were collected over a course of 3 days and tested using a standard plaque assay 
ML-29. VeroE6 cells, MDCK cells, and intestinal adenocarcinoma cell line, Caco-2, were infected with an MOI of 0.1 PFU/cell of ML-29 (Fig. 6). Here, ML-29 shows poor growth kinetics in MDCK cells, reaching peak titers of only $1 \mathrm{X} 10^{2}$, barely above the limit of detection for plaque assays. However, ML-29 replicates exceedingly well in VeroE6 cells, reaching titers of $1 \times 10^{6}$. Additionally, replication of ML-29 was successful in Caco-2 cells as well, reaching titers close to $1 \times 10^{5}$. Due to the replication kinetics of ML-29 in these three cell types, we pursued Caco-2 cells as a model for mimicking the interaction of arenaviruses at the level of epithelial tissue.

\section{Infection of Polarized Caco-2 Cells with OW Arenaviruses Does Not Affect}

the Monolayer Integrity. Since the gastrointestinal tract likely plays an essential role in the arenavirus rodent-to-human transmission, we used the human adenocarcinoma Caco-2 cell line to establish an in vitro model of the intestinal epithelia lining of the gut (Figure 7 A) [167]. The formation of a polarized monolayer was assessed by TEER and by detection of the tight junction protein Zonal-Occludin-3 (ZO-3), a partition marker of the apical and basolateral sides of the cells, was readily detected in between sister cells (Figure 7 B). Furthermore, polarized Caco-2 were stained with antibody against $\alpha-D G$, a principal cell receptor for OW arenaviruses (Figure 7 B).

In general, the arenavirus species used in this study are not associated with cytopathic effects. Nonetheless, it was essential to the utility of our model to confirm that the apical and basolateral separations were intact during infection. In 

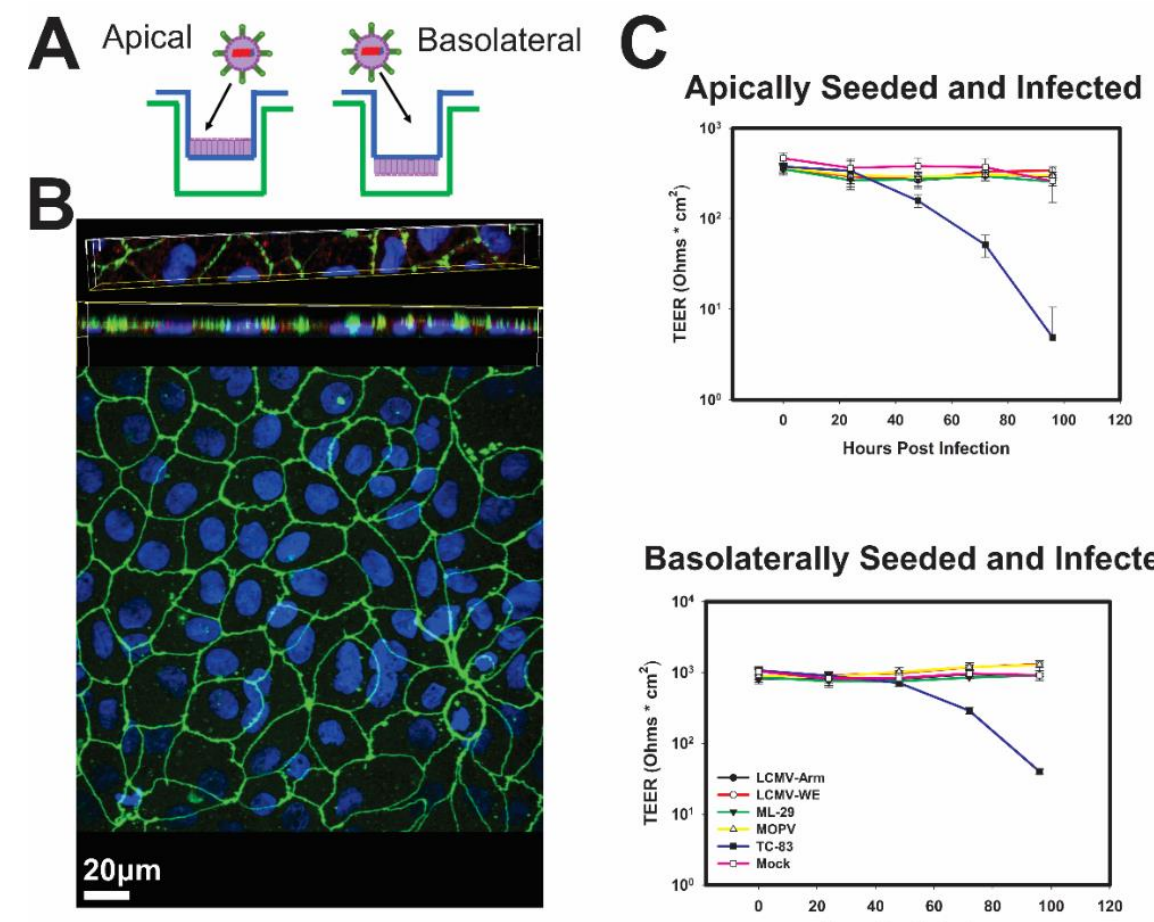

Basolaterally Seeded and Infected

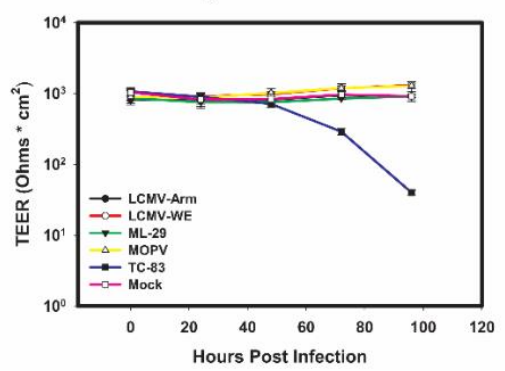

Figure 7. Old World (OW) mammarenaviruses do not alter integrity of model intestinal epithelia during infection.

(A) Diagram of Caco-2 cell seeding for apical infection (left) or basolateral infection (right) during polarization; (B) After 21-day polarization period, Caco-2 cells form confluent monolayers with markers of polarization such as apical tight junction protein ZO-3 (green), and a-DG (red) on the basolateral side of cells; (C) Caco-2 cells were seeded for apical infection (top) or basolateral infection (below) and polarized for 21 days. After which the cells were either Mock infected with PBS, or lymphocytic choriomeningitis virus (LCMV)-Armstrong, LCMV-WE, ML-29, Mopeia (MOPV), or Venezuelan Equine Encephalitis (VEE) virus (vaccine strain TC-83) at an MOI of $0.3 \mathrm{PFU} /$ cell. TEER measurements were taken daily for 5 days. Values shown are the means of 3 replicates, with the error bar representing the standard deviation of the means. 
line with their non-cytopathic nature, the OW arenaviruses used in this study did not negatively affect electric resistance of epithelial monolayers during the 5-day observation period, suggesting that integrity of monolayers was preserved during infection (Figure $7 \mathrm{C}$ ). In contrast, the alphavirus Venezuelan equine encephalitis (VEEV) strain TC-83, which is known to be highly cytopathic, readily disrupted the integrity of polarized Caco-2 cells. Analysis of the mRNA and protein levels of tight junction proteins was tested and no significant change in quantity of tight junction proteins was observed in infected cells as compared to mock infected.

To determine if cellular polarization unexpectedly perturbed the replication of OW arenaviruses, polarized and non-polarized Caco- 2 cells were infected with both strains of LCMV, MOPV, as well as reassortant virus ML-29. The replication kinetics were monitored in both polarized and non-polarized cells by plaque assay. LCMV-Arm (Figure 8 A) and LCMV-WE (Figure 8 B) exhibited similar replication kinetics regardless of the polarization state of the Caco-2 cells. It should be noted that at $24 \mathrm{~h}$ post infection LCMV-WE did show a slight difference in titer between the polarized and non-polarized Caco-2 cells, but these differences were not observed in further time points. As such, neither LCMV-Arm, nor LCMV-WE, infections were significantly impacted by the polarization of Caco2 cells during apical infection (Figure 8). In addition, similar to LCMV, ML-29 was not significantly impacted by polarization of these cells (Figure $8 \mathrm{C}$ ); however, peak viral titers were approximately 2-log lower than those for LCMV or MOPV (Figure $8 \mathrm{D}$ ). Taken together these results indicate that polarization of Caco-2 
A

LCMV-Armstrong

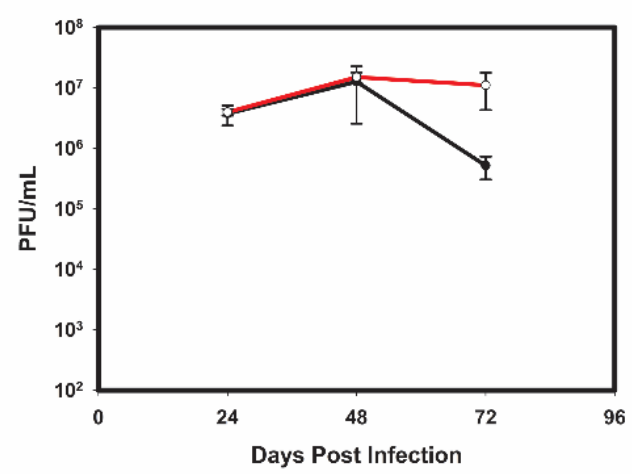

C

ML-29

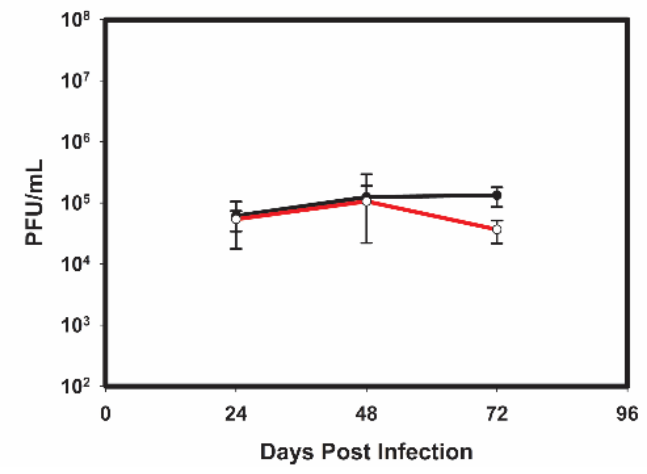

LCMV-WE

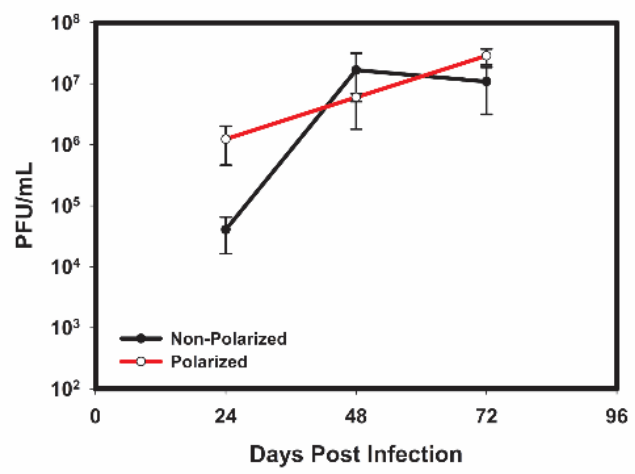

MOPV

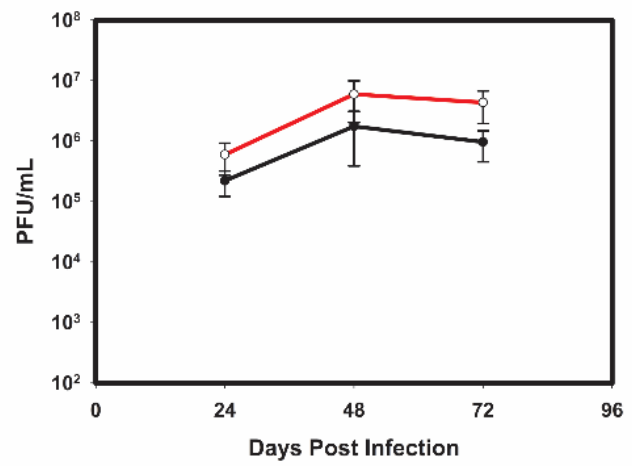

Figure 8 Polarization of Caco-2 cells does not significantly impact OW arenaviral replication

Caco-2 cells were seeded in 96-well plates and polarized for 2 weeks, or plated for 3 days (non-polarized) and infected with either LCMV-Arm (A); LCMV-WE (B); ML-29 (C); or MOPV (D) at an multiplicity of infection (MOI) of 0.3 PFU/cell. Supernatants were collected every $24 \mathrm{~h}$ for a $72 \mathrm{~h}$ period, and virus production was determined via standard plaque assay. Values shown are the means of 3 biological replicates with the error bar representing the standard deviation of the mean. 
has minimal, if any, inadvertent effect on replication kinetics of the OW arenaviruses.

\section{LASV/MOPV Reassortant ML-29 Exhibits Different Viral Entry and Exit Patterns Compared to Either LCMV or MOPV. As described earlier, the} gastrointestinal tract is one of the major gates of arenavirus entry during rodentto-human transmission $[17,45,137]$. During transmission, epithelial barriers may affect pathogenicity of the OW arenaviruses $[155,169]$. To assess the role of the intestinal epithelial barrier during infections of LCMV strains with different pathogenic potential for NHPs, the polarized Caco-2 cells were exposed either apically or basolaterally to the aforementioned OW arenaviruses. To verify the integrity of the polarized monolayer during the experiment, TEER was measured regularly, and the apical and basolateral supernatants were collected every $24 \mathrm{~h}$ for a period of 5 days.

Apical exposure of polarized Caco-2 cells to both strains of LCMV resulted in robust infection and virus release from primarily the apical cell surface. Nonetheless, basolateral release of infectious virus particles was observed at later times post infection. Therefore, while infectious particles were released from both surfaces, the release was more efficient from the apical surface, with a 2log difference between the two supernatants (Figure 9 A). In contrast, when polarized Caco-2 cells were exposed to LCMV-Arm and LCMV-WE via the basolateral side, infection resulted in roughly the equivalent release of infectious particles from both cell surfaces (Figure $9 \mathrm{~B}$ ). 

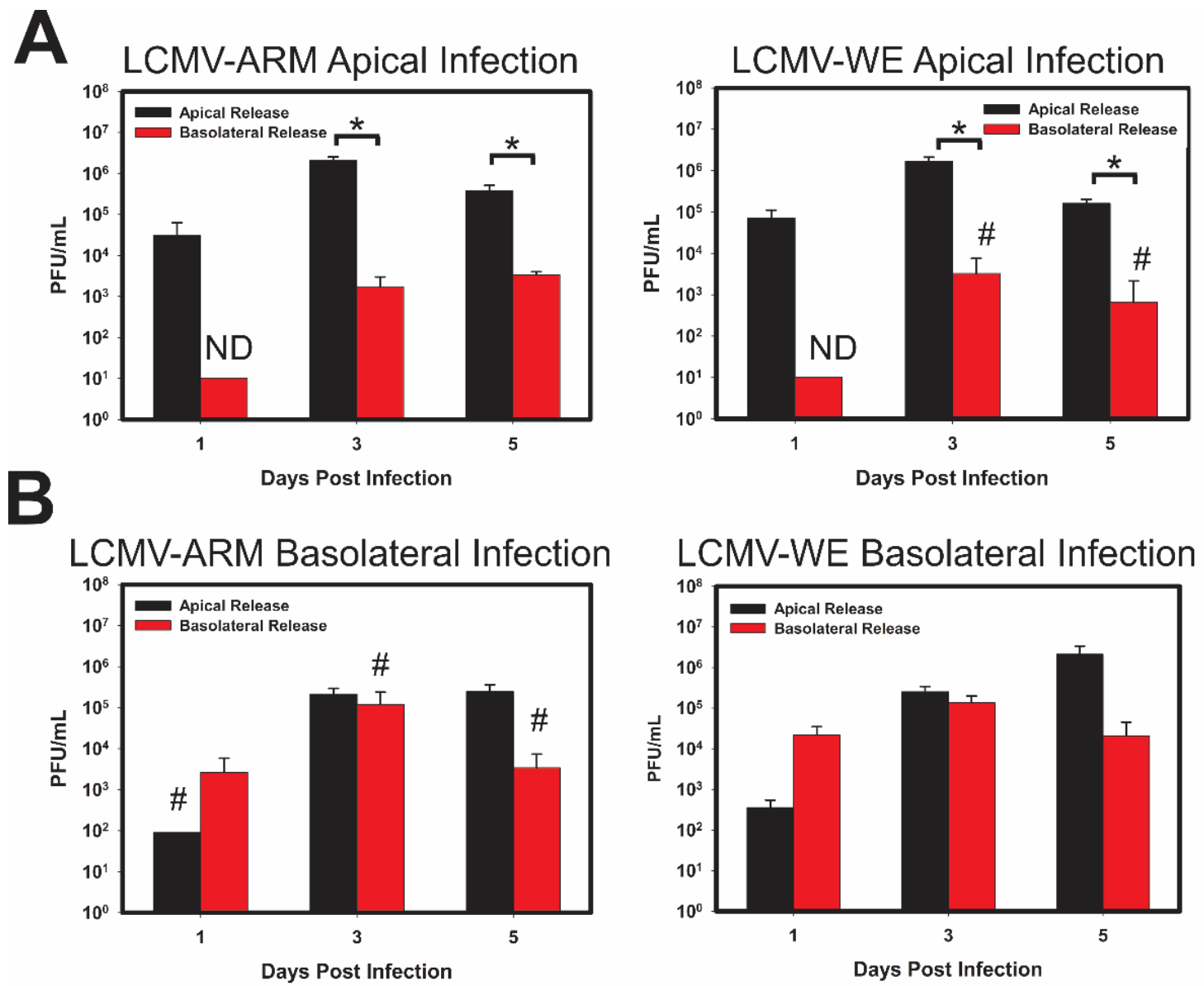

Figure 9 LCMV-Armstrong and LCMV-WE show similar patterns of entry and release in polarized Caco-2 cells regardless of pathogenic differences.

Caco-2 cells were polarized on $0.4 \mu \mathrm{m}$ Transwell inserts in apical or basolateral orientation, for 21 days. After integrity of the monolayer was verified using TEER, the cells were infected with either LCMV-Arm, LCMV-WE, at an MOI of 0.3 PFU/cell on either the apical (A); or basolateral (B) side of polarized Caco-2 cells. Supernatants were collected from both the insert, and well of the Transwells, to determine viral release from the apical or basolateral surfaces independent from one another. Viral titer was measured using standard plaque assay. Release from the Apical surface (black) and the Basolateral surface (red) is plotted with respect to time, with initial viral load subtracted. Values shown are the means of 
3 biological replicates with the error bar representing the standard deviation of the mean. If viral plaque forming units (PFUs) were not observed, data received a place-holder value to signify samples were tested, but no data (ND) was collected. \# indicates that one or more biological replicates was below limit of detection. ${ }^{*} p$-value $\leq 0.05$ 
A

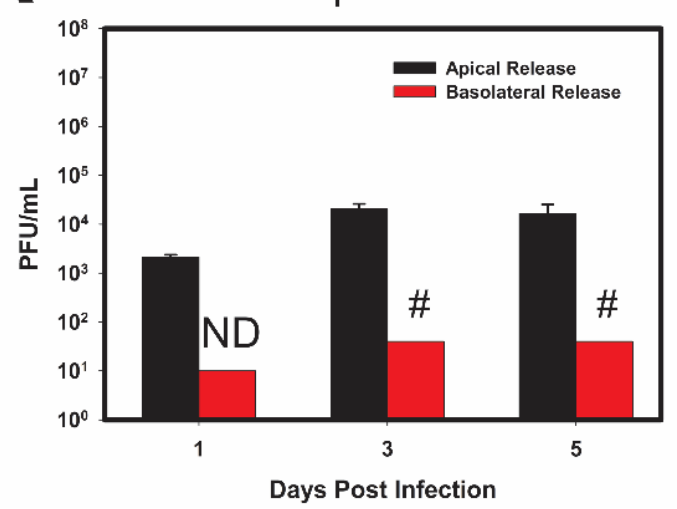

B ML-29 Basolateral Infection

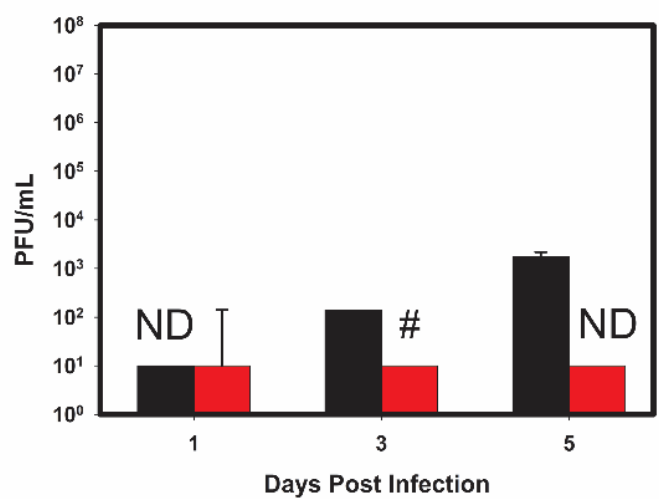

Figure 10. ML-29 replication in polarized Caco-2 cells differ from the replication patterns of LCMV.

Caco- 2 cells were polarized on $0.4 \mu \mathrm{m}$ Transwell inserts as stated for Figure 9. Cells were then infected on the apical $(A)$ or the basolateral $(B)$ surface of the cells with an $\mathrm{MOI}$ of $0.3 \mathrm{PFU} /$ cell of reassortant vaccine candidate ML-29.

Supernatants were collected from transwells and inserts to determine viral release from the apical or basolateral surfaces independent of one another, on every day for 5 days. Release from the Apical surface (black) and the Basolateral surface (red) is plotted with respect to time, with initial viral load subtracted.

Values shown are the means of 3 biological replicates with the error bar representing the standard deviation of the mean. If viral PFUs were not observed, data received a place-holder value to signify samples were tested, but no data (ND) was collected. \# indicates that one or more biological replicates was below limit of detection. * $p$-value $\leq 0.05$. 
In addition to the two strains of LCMV described above, the patterns of viral entry and release of ML-29 was assessed in the polarized Caco-2 model. As shown in (Figure 10 A) Caco-2 cells apically infected with ML-29 failed to produce detectable virus particles from the basolateral surface, despite the apparent release of infectious viral particles from the apical side. Comparative analysis indicates a 2-3-fold difference between viral apical and basolateral release during apical ML-29 infections of polarized Caco-2 cells. Curiously, infection of the polarized Caco-2 cells via the basolateral side resulted in only apical release (Figure $10 \mathrm{~B}$ ). Notably, the release of infectious ML-29 progeny were temporally delayed during basolateral infections, and resulted in the formation of low viral titers.

Parallel analysis of MOPV infection reveals a pattern of viral release similar to that observed for LCMV. As shown in (Figure $11 \mathrm{~A}$ ), the apical infection of polarized Caco-2 cells primarily resulted in the release of infectious viral particles from the apical surface; however, basolateral release was observed. Similar to LCMV, and different from ML-29, basolateral infection of polarized Caco-2 cells resulted in the more-or-less equivalent release of viral progeny apically and basolaterally. A summary of entry and exit kinetics can be seen in Figure 12.

\section{Attachment and Binding of OW Arenaviruses to Polarized Caco-2 Cells.}

To assess if the aforementioned OW arenaviruses differed in their capacity to attach/bind to intestinal epithelia, polarized Caco-2 cells were infected 

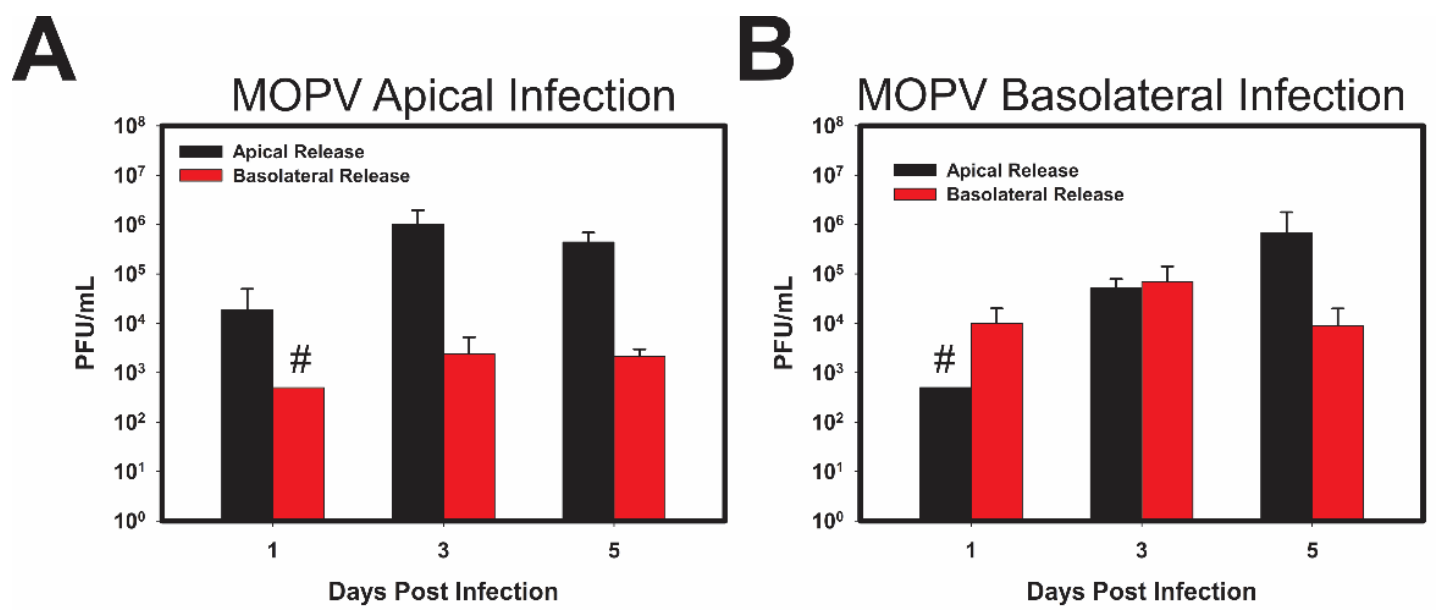

Figure 11. Mopeia virus replication in polarized Caco-2 cells follows a similar pattern as LCMV replication.

Caco-2 cells were polarized on $0.4 \mu \mathrm{m}$ Transwell inserts in apical or basolateral orientation, for 21 days. After integrity of the monolayer was verified using TEER, the cells were infected with MOPV, at an $\mathrm{MOI}$ of $0.3 \mathrm{PFU} /$ cell on either the apical (A) or basolateral (B) side of polarized Caco-2 cells. Supernatants were collected from both the insert, and well of the Transwells, to determine viral release from the apical or basolateral surfaces independent from one another. Viral titer was measured using standard plaque assay. Release from the Apical surface (black) and the Basolateral surface (red) is plotted with respect to time, with initial viral load subtracted. Values shown are the means of 3 biological replicates with the error bar representing the standard deviation of the mean. If viral PFUs were not observed, data received a place-holder value to signify samples were tested, but no data (ND) was collected. \# indicates that one or more biological replicates was below limit of detection. 

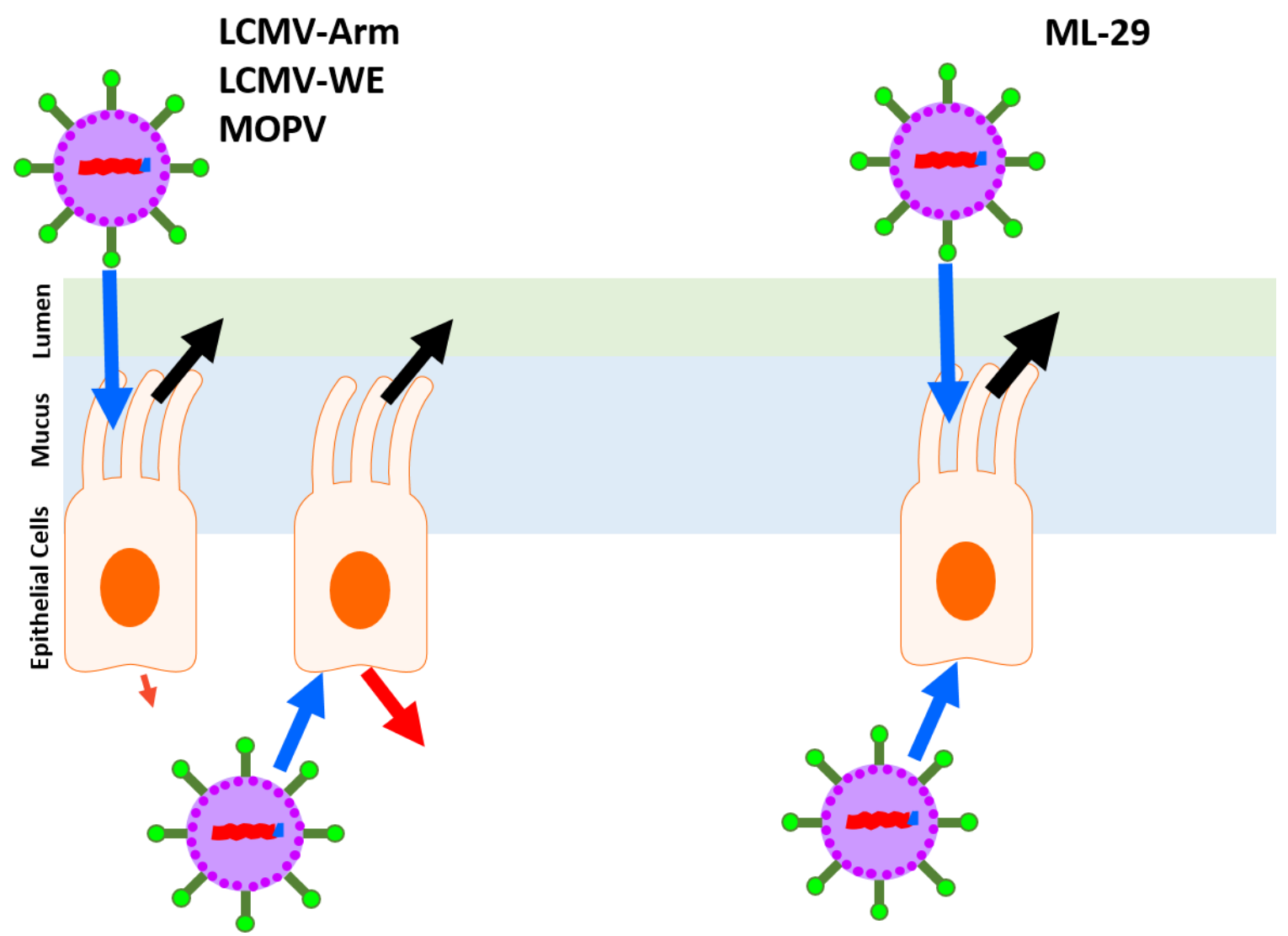

Figure 12. Summary of entry and exit patterns of $O W$ arenaviruses in polarized Caco-2 cells

LCMV-ARM, LCMV-WE, and MOPV entered (Blue arrow) on the apical surface of Caco-2 cells and released primarily to the apical surface (Black arrow) with slight release from the basolateral surface (Red arrow). When exposed to the basolateral surface, LCMV and MOPV released from both the apical (Black arrow) and basolateral (Red arrow) surfaces of the cells. ML-29, when exposed to either the apical or basolateral surface of polarized Caco-2 cells released from the apical (Black arrow) surface of the epithelia. 
at an equal $\mathrm{MOI}$ (0.3 PFU/Cell), via the apical or basolateral surfaces. Cells were incubated at $4{ }^{\circ} \mathrm{C}$ for $1 \mathrm{~h}$ to allow virus to attach, but not penetrate the host cell. To determine the relative attachment rates of the viral particles, the total RNA was extracted from unwashed and washed tissue culture cells of at least three biological replicates derived from at least two independently generated viral stocks. The relative abundance of viral RNA was detected by qRT-PCR using virus-specific primers to determine the percent of input of virus particles that bound to the polarized Caco-2 cells.

Analysis of LCMV attachment indicated that for both LCMV-ARM and LCMV-WE $\sim 5 \%$ of the input virus adsorbed to the polarized Caco-2 cells (Figure $13 \mathrm{~A}, \mathrm{~B})$. Comparisons of apical- and basolateral-bound viral levels indicated that LCMV-ARM exhibited preferential binding to the basolateral surface of the polarized Caco-2 cells (Figure 13 A). LCMV-WE, in contrast, did not exhibit preferential binding to either surface (Figure $13 \mathrm{~B}$ ). Collectively, these data indicate a potential difference between the two LCMV strains in regards to cell attachment. Assessment of ML-29 binding indicated a strong attachment preference to the apical surface of the cells. As shown in (Figure $13 \mathrm{C}$ ), approximately 4 -fold more virus attached to the apical surface relative to the basolateral surface of polarized Caco-2 cells. These data are in apparent congruence with the observations reported in Figure 4, where basolateral infection were less efficient as compared to parallel apical infections. Quantitative analysis of MOPV attachment reveals similar observations to LCMV-WE. 
LCMV-ARM Attachment to
Polarized Caco-2 Cells

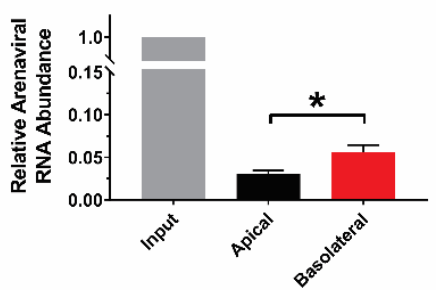

B

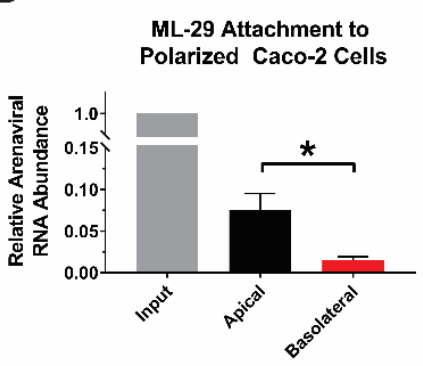

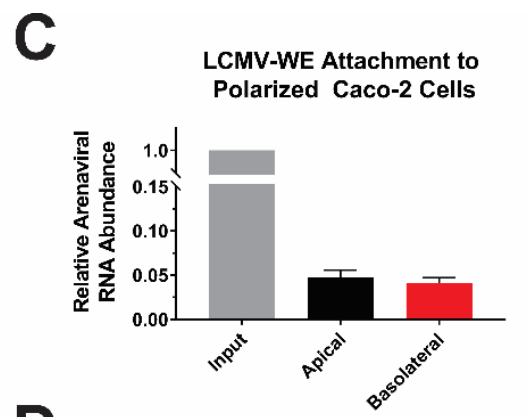

D

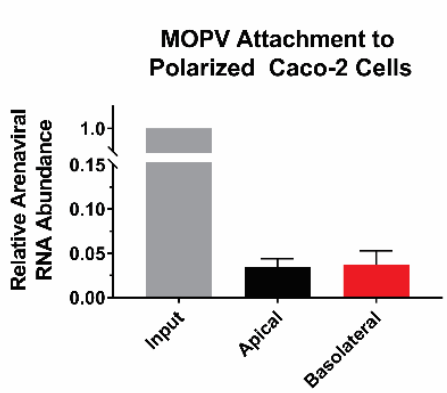

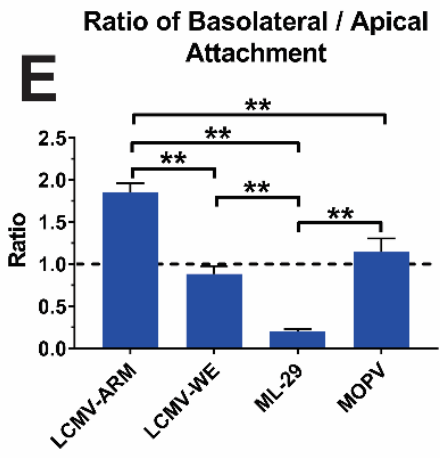

Figure 13 Attachment of ML-29 on the basolateral surface of polarized Caco-2 cells is significantly lower than apical attachment

Caco-2 cells were polarized on $0.4 \mu \mathrm{m}$ Transwell inserts for 21 days in either apical or basolateral orientation. Cells were then infected with an $\mathrm{MOI}$ of 0.3 PFU/cell of LCMV-Arm (A); LCMV-WE (B); ML-29 (C); or MOPV (D) at $4{ }^{\circ} \mathrm{C}$ for 1 $\mathrm{h}$ to allow virus to attach to the epithelial cell surfaces, but not enter the cells. After $1 \mathrm{~h}$, cells were either unwashed (input) or washed and cellular RNA and supernatants were collected for quantitative real-time polymerase chain reaction analysis. Ratio of basolateral to apical attachment was taken by dividing basolateral $\Delta \Delta C T$ values of washed cells by the apical $\Delta \Delta C T$ values of washed cells (E). ${ }^{*} p$-value $\leq 0.05,{ }^{* *} p$-value $\leq 0.01$. 
Specifically, as shown in (Figure $13 \mathrm{D}$ ) the attachment of MOPV virus was equivalent amongst the apical and basolateral surfaces.

Since we cannot directly compare the absolute levels of attachment for each surface between the individual virus species, we must compare the ratios of apically and basolaterally bound viruses to identify statistical differences between the four OW arenaviruses used in this study. Overall, these comparisons indicate that LCMV-ARM and ML-29 differ from the other viruses, and each other, in regards to their binding proclivities. LCMV-Arm has a ratio of apical and basolateral attachment of approximately 1.5 , indicating that attachment has a slight preference to the basolateral surface of the polarized Caco-2 cells (Figure $13 \mathrm{E})$. The ratio of basolateral and apical attachment of LCMV-WE and MOPV of approximately 1 , indicates that LCMV-WE and MOPV attachment is more or less equivalent in its binding to the apical and the basolateral surfaces of Caco-2 cells (Figure $13 \mathrm{E}$ ). While LCMV-ARM is unique in its binding preference as compared to the other viruses, this indicates that LCMV-ARM, LCMV-WE, and MOPV all attach to some degree on the apical and basolateral surface of the cells. However, the ratio of basolateral and apical attachment of ML-29 indicates that there is a large difference between viral attachment between the two surfaces, with a significant preference for the apical side of the polarized Caco-2 cells (Figure 13 E). ML-29's preference for the apical surface with comparatively little to no binding on the basolateral surface, is significantly different than that of LCMV and MOPV. Collectively, these data indicate that LASV-GP has a 
significantly different binding efficacy to the basolateral side of Caco-2 monolayers as compared to LCMV and MOPV.

\section{DISCUSSION}

Epidemiological observations in West Africa indicate that the ingestion of food contaminated with excreta of infected $M$. natalensis is one of the natural mechanisms of LASV transmission to humans [17]. Up to $45 \%$ of individuals living in some LASV endemic regions in Western Africa can be seropositive to the virus; and if re-infection occurs, seropositive individuals can protect themselves from disease onset. However, seronegative individuals may also be protected from disease, due to protection associated with cell-mediated immunity [147]. This implies high prevalence into endemic populations; however, the precise mechanisms behind this phenomenon are unknown. In NHP studies of arenavirus disease, LCMV-WE causes LF-like disease via intravenous (i.v.)infection, whereas LCMV-ARM shows chronic infection, with no disease onset [155]. Furthermore, high titer i.v. infections of NHPs with LCMV-WE resulted in uniform mortality [155]. Nevertheless, i.g. infection with identical titers showed a variable outcome, ranging from no signs and symptoms to fatal, LF-like disease with elevated aspartate and alanine aminotransferases (ALT/AST) levels [155, 182]. Elevated ALT and AST levels are symptomatic of hepatic tissue damage, and highly elevated levels are associated with lethal LF disease of humans in endemic regions [96]. In addition, some of the surviving animals survived i.v. challenge, indicating that LCMV infection did occur as a competent humoral 
response was induced [182]. Due to probable infection via the ingestion of contaminated food stuffs, and the variable outcomes of intragastric infection in vivo, we sought to investigate whether or not the patterns of entry and exit when polarized Caco-2 cells were exposed to OW mammarenaviruses apically (intragastric route) or basolaterally (intravenous route). Due to the unsuccessful infections of LCMV and ML-29 in HBE and MDCK cells, we did not further pursue these cell lines, resulting in our focused studies of the interaction of Caco-2 cells and OW mammalian arenaviruses.

As previously described, LASV and LCMV entry into MDCK cells and HBE cells occurs primarily via the basolateral side, and release of viral particles was predominantly from the basolateral surface. These in vivo and in vitro studies led us to investigate the role of the intestinal epithelial barrier during OW arenavirus infection. In this study, we characterized an in vitro model of intragastric infection to assess the interaction of OW mammarenaviruses with the intestinal epithelia in an amenable tissue culture system. This system utilized polarized Caco-2 cells grown on transwells, which is a well-established cell type and cell culture system used for in vitro studies of the intestinal barrier $[173,187]$. This system enables the independent examination of the role of the apical and basolateral epithelia surfaces during viral infection. Therefore, this model recapitulates the infection of intestinal epithelial cells from the luminal and laminal sides of the epithelial monolayer via the apical and basolateral surfaces, respectively.

Using this model system, we evaluated infections of LCMV strains of different pathogenic potentials. We also used a MOPV/LASV reassortant, ML-29, 
a validated BSL2 surrogate model that is capable of mimicking the interaction of LASV with susceptible cells. ML-29 expresses GP1 attachment glycoprotein identical to LASV GP1, and MOPV, attenuated genetic relative of LASV. Since ML-29 is a reassortant virus of LASV/MOPV, it represents a better system in which arenaviral biology can be determined compared to VSV or retrovirusbased pseudotypes expressing LASV GPC as the viruses contain genuine arenaviral replication machinery.

From the in vivo studies in NHPs, it was expected for virus to release infectious particles from primarily the apical side, since viral particles were not detected in any tissues after intragastric infection with LCMV [155]. However, when exposed on the apical side of epithelial cells, LCMV and MOPV primarily released on the apical side of the cells, but release basolaterally was observed. This observation indicates that the epithelial barrier is not the sole determinant of viral dissemination. Interesting to note as well, was that patterns of replication were similar regardless of the in vivo pathogenicity of the LCMV strains. Therefore, the infectious capacity of these viruses in vitro, in regards to Caco- 2 cells, does not correlate with pathogenic properties for LCMV. Therefore, further investigation as to the driving forces of pathogenic differences needs to be investigated. However, in contrast to LCMV, the ML-29 expressing LASV GP1, predominantly released viral particles apically regardless of the route of entry. Furthermore, these results demonstrate that MOPV entry-release pattern in polarized Caco-2 cells resembled those in cells infected with LCMV, and was clearly different from ML29-driving entry-release. Due to ML-29 replication 
patterns being different than that of MOPV replication, these patterns of entry and release are not due to MOPV replication machinery, and may be attributed to the gene products of the S segment of LASV, namely the glycoproteins and nucleoprotein. This poor replication and egress to the basolateral sides of the cells is an interesting observation. While ML-29 is not WT-LASV, using WT-LASV in similar studies could lead to an explanation as to why almost half of the population of endemic regions are seropositive for LASV, but never demonstrated clinical signs of disseminated illness. In addition, the data presented here indicates that the capacity to infect via the apical surface of intestinal epithelial cells is not a primary determinant of arenavirus pathogenesis.

ML-29 was developed as a potential vaccine for the prevention of LASV infection. As seen here, ML-29 had a much greater binding rate leading to successful infection when exposed to the apical side of polarized intestinal epithelia. If after successful attachment following the ingestion of viral particles fails to release viral particles basolaterally, this may be an exceptionally potent tool for the development of successful immunity against LASV in at-risk populations. These results provide additional evidence for attenuation of ML-29 as a vaccine strain for LASV. From these in vitro studies, and the dire need for a LASV vaccine, studies examining the importance of the route of exposure to ML29 in an in vivo model should be investigated as a potential therapy to LASV infection and prevention.

A recent publication from Oppliger et al. identified entry of a recombinant LCMV expressing LASV-GP (rLCMV-LASVGP) in polarized Caco-2 cells [190]. 
Here, rLCMV-LASGP showed preferential entry into polarized Caco-2 cells on the basolateral surface of these cells. Opposingly, we identified via qRT-PCR that viral attachment of ML-29, a reassortant containing the GP1 of LASV, preferred the apical surface of polarized Caco-2 cells. Interestingly, we did see LCMV preferentially attaching to the basolateral surface of polarized cells, as seen with rLCMV-LASVGP. However, the rLCMV-LASGP studies did not elucidate the viral release patterns of rLCMV-LASVGP in polarized Caco-2 cells, nor the initial attachment of viruses to these cell surfaces. Furthermore, due to ML-29's genetic differences to the LCMV-backbone of rLCMV-LASVGP, our results and those of Oppliger et al. cannot be directly compared. Taken together, our observations, are a useful addition to the field to further investigate precise differences between rLCMV-LASVGP and ML-29 in order to evaluate genetic variations of these viruses to more accurately identify potential targets for LASV therapeutics and further understand the replication cycle of LASV.

Some questions still lie as to the reason that ML-29 bound so inefficiently on the basolateral side of Caco-2 cells. Although primary receptor ( $\alpha-D G)$ is located on the basolateral surface of the polarized Caco-2 cells, this data, along with the data of others, provides further support that LASV has complex receptor usage. Additional receptors for LASV should be investigated in the Caco-2 system, including AxI, DC-SIGN and Tyro3. Along with additional receptors and their role in our system, the interaction with $\alpha-D G$ should also be investigated. An excellent review by Torriani et al. describes a number of studies that explain the complex viral-receptor interaction of LASV [191]. Although fully functional $\alpha-D G$ 
was detected basolaterally in polarized Caco-2 cells, ML-29's binding efficiency was low. This may be due to a multitude of reasons including the use of other cellular factors and receptors used in addition to $\alpha-D G$ to attach to these polarized cells, or differences and mutations that ML-29 may contain as compared to WT LASV, especially those present in the GP2 protein. Previous research has identified that LASV infection was dependent upon sodium hydrogen exchangers (NHEs), as well as actin cytoskeleton to have successful viral entry into host cells [40]. Investigations into these factors during infection of polarized Caco- 2 cells should be analyzed to determine a reason for inefficient and poor binding of ML-29 on the basolateral side of these cells. Although ML-29 contains the $S$ segment of LASV, the L segment of MOPV may interfere with complete and successful viral replication that WT LASV may have, comparatively. However, we believe the latter to be a minimal or insignificant inhibition of viral attachment and replication due to MOPV replicating similarly to LCMV in polarized Caco-2 cells when exposed to the basolateral side of the cells, as compared to little or no viral replication by ML-29 after basolateral exposure. Thus, investigation into precisely how WT LASV attaches, enters, and releases from polarized Caco-2 cells would be a valuable addition to the field.

\section{CONCLUSIONS}

To conclude, our data above demonstrates that the polarized Caco-2 system is a viable model to investigate the interaction of intestinal epithelial cells during viral infection with OW mammarenaviruses. These polarized epithelia 
closely mimic intestinal epithelial of human hosts and allow further investigation of mammarenaviral infection at the epithelial barrier. These data with LCMV show that intestinal epithelial cells may not be the sole determinant of viral pathogenesis and dissemination. Furthermore, differences between prototypic arenavirus LCMV and the surrogate model of LASV interaction, ML-29, were observed in both attachment efficiency and viral entry and egress from polarized intestinal epithelia. These results may potentially explain the high penetrance without disease observed for LASV. In addition, ML-29's diminished binding efficiency to the basolateral side of polarized Caco-2 cells supports the expanding complexity of arenavirus receptor interactions. Collectively, these studies show that arenaviral infection of polarized cells is not only viral specific, but ultimately may be tissue and host-specific as well; and that arenavirus infection and pathogenesis may be dependent on asymmetric distribution of viral and cellular factors required for virus entry and budding. 
CHAPTER 3

\section{STRAIN-SPECIFIC INTRACELLULAR TRAFFICKING OF LCMV, A PROTOTYPIC MAMMALIAN ARENAVIRUS}

Wang, $\mathrm{M}^{\#}$, Warner NL\#, Jokinen, JD, Lukashevich, IS. Strain-specific intracellular trafficking of LCMV, a prototypic mammalian arenavirus. Viruses 2018. Under Review.

\# denotes co-first authorship 


\section{OVERVIEW}

Lymphocytic choriomeningitis virus (LCMV), prototype of rodent-borne arenaviruses, and Lassa virus (LASV), causative agent of the most prevalent hemorrhagic fever in West Africa Lassa Fever (LF), share many genetic and biological features. Both species comprise of a collection of highly diverse, genetically and biologically, virus isolates from rodent hosts and humans. Pantropic LCMV-WE causes fatal LF-like hepatitis in non-human primates (NHPs); while heavily adapted in mice, LCMV-ARM strain, is deeply attenuated in NHPs and can protect animals against fatal WE challenge. Similarly, Mopeia virus (MOPV), a genetic relative of LASV, causes asymptomatic infection in NHPs, and protects them against fatal LF. Previously we demonstrated that nonpathogenic MOPV and LCMV-ARM (but not LASV and LCMV-WE) induced robust Toll-like receptor 2 (TLR2)/Mal (MyD88 adaptor-like)-dependent and NFKB-mediated cytokine responses. These responses correlated with virus replication. In this study, we demonstrate that LCMV strains with different pathogenic potential had distinct intracellular trafficking patterns in macrophages. After internalization, LCMV-ARM strongly interacted with TLR-2, and markers of early and late endosomes where nucleic acid-sensing TLR-7 and-9 are located. In contrast, LCMV-WE bypassed the early endosomal compartment, and had less extensive interaction with late endosomes/lysosomes markers as assessed by co-staining experiments by confocal microscopy. LCMV-WE NP antigen was strongly co-localized with IRAK-1 and can affect NF-KB-mediated signaling. Internalization of LCMV-WE in Vero and Caco-2 cells was more sensitive to 
depletion of membrane cholesterol and disruption of microtubules in polarized epithelial cells than LCMV-ARM infection. These findings suggest that nonpathogenic LCMV-ARM infection is more efficient in terms of activation of TLRsand RIG-I-mediated signaling driving effective innate and adaptive immune responses.

\section{INTRODUCTION}

Lymphocytic choriomeningitis virus (LCMV) is a prototypic virus of the Arenaviridae family, which was dramatically reshaped after the discovery of snake-borne viruses. Until recently, all arenaviruses have been placed into one genus, Arenavirus, and were divided into two groups based on geographical locations and serological relationships; Old World (OW) arenaviruses (or LCMVLASV sero-complex) and New World (NW) arenaviruses (or Tacaribe, TCRV, sero-complex). Lassa virus (LASV) is the most prevalent human pathogen among OW arenaviruses, infecting hundreds of thousands of individuals annually in West Africa, some of them resulting in fatal Lassa fever (LF). Junin virus (JUNV), causative agent of Argentine hemorrhagic fever (AHF), is the most significant human pathogen among NW arenaviruses. All arenaviruses share common features as enveloped RNA viruses, with a two-segmented, singlestranded, ambisense genome. The large (L) RNA, encodes $L$ protein (RNA polymerase) and matrix Z protein. The small (S) RNA encodes the most abundant protein, nucleocapsid protein (NP), which is tightly associated with RNA in virions and in infected cells. Additionally, $S$ encodes the enveloped glycoprotein precursor (GPC) protein, which is processed in infected cells into 
stable signal protein (SSP), GP1 (attachment), and GP2 (fusion) glycoproteins [192]. Intergenic region with extensive secondary structure separate the genes in both RNA segments, and is required for transcription and replication.

In 2014, rodent-borne arenaviruses, comprising a single Arenavirus genus, were placed in the renamed genus Mammarenavirus. A new genus, Reptarenavirus, was established for newly discovered arenavirus-like viruses isolated from alethinophidian snakes [3]. Recently, eight novel species were included in the genus Mammarenavirus for murid viruses isolated in Africa and Asia. The genus Hartmanivirus was created to accommodate a novel arenavirus isolated from a captive snake in Finland [3]. In addition, the Arenaviridae family was placed into the newly established order Bunyavirales for related viruses with single-stranded negative-sense (or ambisense) RNA genomes [193].

LCMV and LASV share biological features including their interaction with major cellular receptor $\alpha$-dystroglycan ( $\alpha-D G)$ [10], and pathogenicity in guinea pigs and non-human primates (NHPs) [194, 195]. Both species, Lymphocytic choriomeningitis mammarenavirus and Lassa mammarenavirus, comprise a collection of highly diverse (genetically and biologically) virus isolates from rodent and humans, phylogenetically placed into 4-6 lineages [196-201]. The unbiased pairwise sequence comparison (PASC) analysis split LASV and LCMV strains into 6 and 5 distinct species, respectively. A conservative PASC cut-off (>80\% and $>76 \%$ nucleotide sequence identity in the $S$ and $L$ segments, respectively, to belong to the same species) was proposed to leave the current taxonomy of mammalian arenaviruses intact [3]. 
LASV causes infection with broad clinical manifestations, from sub-clinical or flu-like disease, to fatal LF disease and is found in endemic areas of West Africa. In contrast, LCMV is widely spread across continents and causes asymptomatic or mild infections, which rarely progress to aseptic meningitis or meningo-encephalitis $[128,202]$. However, during the third trimester of pregnancy, a relatively immune-suppressed state, both viruses, LASV and LCMV, can cause infection with disastrous consequences for the fetus [128, 203]. Furthermore, in immunocompromised recipients that received LCMVinfected organs, the virus can cause fatal LF-like infection [113, 114].

LASV and genetically related non-pathogenic Mopeia virus (MOPV), are hosted by the same rodent species, Mastomys natalensis, and can produce interspecies reassortants in vitro after co-infection cells with both viruses. One of these reassortants, clone ML29, carrying the L RNA from MOPV and S RNA from LASV, is a promising LF vaccine candidate [85]. Similarly, pantropic LCMVWE strain, causing LF-like fatal hepatitis in non-human primates (NHPs) [153, 156], can produce reassortants in vitro with neurotropic strain LCMV-ARM [204], which is highly adapted in murine cells and fully attenuated in NHPs. For both types of reassortants, interspecies MOPV/LASV and intertypic WE/ARM, the pathogenic potential for humans and NHPs was linked to the L RNA encoding RNA polymerase [204, 205]. Furthermore, LCMV-WE is highly pathogenic for outbred guinea pigs ( $\mathrm{LD}_{50}<\sim 1$ PFU), while mouse-adapted LCMV-ARM is fully attenuated in these animals ( $>6 \log _{10}$ PFU failed to kill) [194]. 
The immunosuppressive phenotype of LASV infection contributes to fatal outcomes in progressed cases of human LF disease, and in experimentally infected NHPs [206, 207]. We previously documented that LASV and LCMV-WE, but not MOPV and LCMV-ARM, down-regulated innate pro-inflammatory responses in vitro and in vivo $[87,102,153,156,167]$. Particularly, cells infected with LASV and LCMV-WE, inhibited Toll-like receptor 2 (TLR2)/Mal (MyD88 adaptor-like)-dependent cytokines [167]. In contrast, MOPV and LCMV-ARM, induced robust NF-kB-mediated, Mal-dependent pro-inflammatory responses in human epithelial cells, monocytes, and in murine bone marrow-derived macrophages [167]. These responses were TLR2/Mal-dependent, required virus replication, and were enhanced by CD14 [167]. Virus internalization and virus replication was required for activation of TLR2/Mal-dependent signaling [167].

Strong activation of monocyte-macrophages seems to be a general feature of non-pathogenic mammalian arenaviruses [102, 158-162]. In addition to LCMV-ARM and MOPV, TCRV, but not pathogenic JUNV, induced cytokines release from these cells [158]. With these similarities, TLR2/Mal/MyD88dependent signaling has some differences between the OW and NW mammalian arenaviruses. In the case of Candid 1 (attenuated JUNV vaccine), engagement of TLR2/6 heterodimers on the cell surface, with viral glycoprotein, was sufficient to trigger cytokine response via RIG-I/MDA5, NF-kB, and Erk1/2 pathways and did not require internalization and viral replication [55].

In the virus overlay protein-binding assay (VOPRA), LASV and LCMV-WE bound to its major cellular receptor, $\alpha-D G$, with higher affinity and efficiency than 
non-pathogenic Mobala virus (MOBV) (which is genetically closely related to MOPV), and LCMV-ARM $[10,13]$. However, while both LCMV strains, WE and ARM, replicated with the similar kinetics in human monocyte derived cells, only the replication of LCMV-ARM resulted in robust TLR2/Mal-dependent proinflammatory cytokine responses [167]. Interestingly, attachment efficacy of infectious particles of both strains of LCMV, as well as patterns of entry and exit, were similar in polarized epithelial Caco-2 cells [208]. In line with these observations, LCMV strains with high and low bidding affinity to $\alpha-D G$, infect BHK-21 cells with equal efficiency [209]. Additional entry factors recently discovered in vitro for LASV and LCMV (DC-SIGN, LSECtin, Axl, and Tyro3) seem play a role in virus entry $[30,31,210,211]$. At least one of these factors, $A x l$, was strongly upregulated in maturated hepatocytes lacking functional a-DG (O-mannosyl glycosylated) in mice infected with LCMV-WE (but not in LCMVARM) [212].

Taken together, these facts indicate that the engagement of receptors, and/or attachment cofactors, on the cell surface by viral glycoproteins cannot be solely responsible for trigger (or suppression) of virus-induced pro-inflammatory responses. All consequential steps (internalization, intracellular trafficking and cross talk with cellular factors, fusion in late endosomes/lysosomes, and triggering of RIG-I-dependent signaling), can differently contribute to innate and adaptive immune responses driving pathogenicity. In this study we have compared intracellular trafficking of LCMV-WE and LCMV-ARM using co-staining with markers of early and late endosomal compartments and treatment of cells 
with drugs targeting different steps of virus replication. In LCMV-ARM-infected macrophages viral NP antigen was extensively co-localized with TLR-2 in early endosomes, and was co-stained with markers of late endosomes where nucleic acid sensing TLR-7 and TLR-9 are located. In contrast, LCMV-WE bypassed the early endosomal compartment, and had less extensive interaction with late endosomes/lysosomes markers as assessed by co-staining experiments and by confocal microscopy. LCMV-WE infection of murine macrophages resulted in strong co-localization of viral NP with IRAK-1 in line with of NF-KB suppression in infected cells [167],

Furthermore, LCMV-ARM infection was more sensitive to $\mathrm{pH}$ changes of late endosome, tested with bafilomycin A1, in comparison to LCMV-WE. These findings are in line with previous observations, documenting high sensitivity of MOPV to $\mathrm{NH}_{4} \mathrm{Cl}$ in comparison to LASV [213]. Taken together, these data suggest that fusion events in late endosomes drives viral RNA release kinetics resulting in the differential triggering of the cellular RIG-I machinery and innate responses.

\section{MATERIALS AND METHODS}

Cells and Viruses. Vero E6 (C1008), Caco-2 (HTB-37), and RAW264.7 (TIB$71^{\mathrm{TM}}$ ) cells were purchased from American Type Culture Collection (ATCC). Caco-2 cells were grown and polarized as previously described [208]. Cells were infected with LCMV-Armstrong (strain 53b) or LCMV-WE (strain 54). All viral stocks were generated using low multiplicity of infection (MOI) and stocks were 
generated ranging from $1 \times 10^{7}$ to $1 \times 10^{8} \mathrm{PFU} / \mathrm{mL}$ and stored at $-80^{\circ} \mathrm{C}$ until further use [88]. Infectious virus titration was performed in Vero E6 cells using a standard plaque assay with minor modifications that have been previously described $[167,208]$. Briefly, VeroE6 cells were plated in 12 well plates. When Vero Cells reached $80-90 \%$ confluent, viral samples were serially diluted and used to infect. Infection was carried out for 1 hour in $37^{\circ} \mathrm{C}$. Cells were then washed with PBS, and a semi-solid overlay containing $1 \mathrm{X}$ MEM, $5 \% \mathrm{FBS}$, and 0.5\% Avicel (FMC BioPolymer, Philadelphia, PA, USA) was added to the cells. Cells were incubated for 5 days in a humidified chamber at $37^{\circ} \mathrm{C}$ and $5 \% \mathrm{CO}_{2}$. After a 5-day incubation, the semi-solid overlay was removed, cells were washed with PBS, and fixed with 4\% paraformaldehyde (PFA). After a 15-minute fixation, cells were stained with a $1 \%$ Crystal Violet solution to identify virus-infected foci, with a limit of detection of approximately $80 \mathrm{PFU} / \mathrm{mL}$.

TLR-2 silencing. RAW264.7 cells were pretreated with TLR2 Silencer® select pre-designed siRNA (Ambion, P/N 4390771). Transfection was performed with Lipofectamine (Lipofectamine RNAi Max Reagent, Invitrogen) for 48 hours. Control cells were transfected with Silencer ${ }^{\circledR}$ Negative Control siRNA (Ambion). Transfection of mock-infected cells with TLR2 siRNA resulted in moderate suppression of TLR-2 mRNA (46\%), and TLR-2 expression on the cell surface as assessed by staining transfected cells with anti-TLR-2 antibody and flowcytometry (36.5\%). Transfected cells were infected with LCMV stains at MOI > 1 and incubated for $24 \mathrm{hrs}$. The mRNA levels of IL- 6 were determined by real-time 
PCR using commercial primers/probe as previously described [167]. Expression of IL-6 at protein level was analyzed by ELISA kit (eBiosciencecat, P/N 88-7064).

Immunofluorescence co-staining, confocal microscopy. RAW264.7 cells were grown in chamber slides (Lab-Tek), and infected with LCMV strains at MOI $>1$. After virus internalization, intracellular trafficking of LCMV infection was monitored in co-staining experiments using monoclonal antibody against a conserved NP epitope (M104, Abcam, 1:100 dilution), TLR-2 (monoclonal CD282, eBioscience, 1:200), EEA1 (monoclonal F.43.1, Thermo scientific, 1:100), RAB-7 (rabbit monoclonal EPR7589, Abcam, 1:100), LAMP1 (polyclone C-20, Santa Cruz, 1:100), IRAK-1 (D51G7, Cell Signaling, 1:250). Infected cells were incubated in $\mathrm{CO} 2$ incubator for $0.5-2$ hrs (see legends to Fig. 15,16,18, and 19 for more details), fixed with PFA and permeabilized. After blocking of nonspecific binding with $1 \%$ BSA, cells were co-stained with primary antibodies (2-4 hrs, RT) and treated with the secondary IgG-TR or IgG-FITC antibodies (antimouse or anti-rabbit, 1:200 dilution for $30 \mathrm{~min}$ at RT). To stain nuclei, DAPI Flu G (Southern Biotech) was used mount coverslips on slides. Co-staining patterns were examined by confocal microscopy (LSM 710, Zeiss) as previously described, and co-localized staining was quantitated (see Supplemental Materials).

Confocal Microscopy. The analysis was performed as recently described for LCMV infected cells using ZEN software [214] with slight modifications. Up-to eight LCMV NP-positive (green cells) cells were marked and compared pixel by pixel with intensity signals from each channel. Background for green and red 
channels was determined based on mock-infected cells staining. Total (greenand red-positive) signals were divided by the sum of green positive pixels and expressed as co-localization efficiency, (\%). The calculation was repeated for individual cells

Treatment with Chemical Inhibitors. Treatment with methyl- $\beta$-cyclodextrin (Mß-CD) was modified from previous work [36, 215, 216]. Briefly, VeroE6 or Caco-2 cells were seeded in 12-well tissue culture plates. Cells were then pretreated in the presence, or absence (control cells), of $5 \mathrm{mM} M \beta-C D$ (SigmaAldrich, St. Louis, MO, USA) for $1 \mathrm{hr}$ at $37^{\circ} \mathrm{C}$. Cells were infected with an MOI of 0.3 PFU/cell, and incubated for $24 \mathrm{hrs}$ in the presence of $\mathrm{M} \beta-\mathrm{CD}$. Percent replication after inhibition was determined by normalizing viral titer after treatment with the drug, compared to infections titer without membrane cholesterol depletion. Treatment with nocodazole was slightly modified from previous studies $[35,43,217]$. Briefly, VeroE6 or Caco-2 cells were pre-treated with $10 \mu \mathrm{M}$ nocodazole for $1 \mathrm{hr}$ at $37^{\circ} \mathrm{C}$. Cells were then washed 2 times with cold PBS, infected with a MOI of $0.3 \mathrm{PFU} /$ cell for $1 \mathrm{hr}$ and then incubated in the presence of nocodazole for $24 \mathrm{hrs}$ at $37^{\circ} \mathrm{C}$. Bafilomycin treatment was also slightly modified $[28,218]$. VeroE6 cells were pre-treated with bafilomycin A1 (Sigma-Aldrich, St. Louise, MO, USA) with concentrations ranging from 10 to $200 \mathrm{nM}$. Cells were infected at an $\mathrm{MOI}$ of $1.0 \mathrm{PFU} / \mathrm{cell}$, at $4^{\circ} \mathrm{C}$ for $1 \mathrm{hr}$, in the presence of bafilomycin and incubated with the drug for $24 \mathrm{hrs}$ at $37^{\circ} \mathrm{C}$. Supernatants were collected for standard plaque assays and cells were collected in RNA-STAT-60 (Tel-Test Inc.,) further RNA isolation according to the manufacturer's instructions. 
Quantitative Real-Time Polymerase Chain Reaction (qRT-PCR) was used to quantitate viral RNA in treated and untreated cells using LCMV strain-specific primers/probe as previously described [208].

Statistical Analyses. Statistical significance was analyzed using 3 biological replicates per experimental time point and treatment concentration, using a Standard Student t-Test. All statistical values of $p \leq 0.05$ were deemed statistically significant.

\section{RESULTS}

\section{TLR-2 knockdown results in down-regulation of IL-6 expression in LCMV-}

ARM-infected murine macrophages. In previous experiments, LCMV-ARM (but not LCMV-WE) infection of cells human derived from murine or human monocytes/macrophages induced strong production of IL-6 [167]. This induction was TLR2/Mal-dependent since murine macrophages generated from TLR-2 or Mal knockout mice failed to induce cytokine responses. In current experiments, we have used the transformed murine macrophage cell line RAW264.7. To prove TLR-2 dependency of IL-6 stimulation in LCMV-ARM-infected cells, RAW264.7 cells were transfected with pre-designed TLR-2 siRNAs, and infected with LCMV strains at MOI > $1 \mathrm{PFU} / \mathrm{cell}$. Induction of IL-6 was assessed at mRNA and protein levels, by RT/PCR and ELISA, respectively.

Consistent with previous study, both LCMV virus strains had similar replication kinetics in RAW cells [167]. However, only LCMV-ARM infection strongly stimulated IL-6 (Fig. 14). Transfection of mock-infected cells with TLR-2 


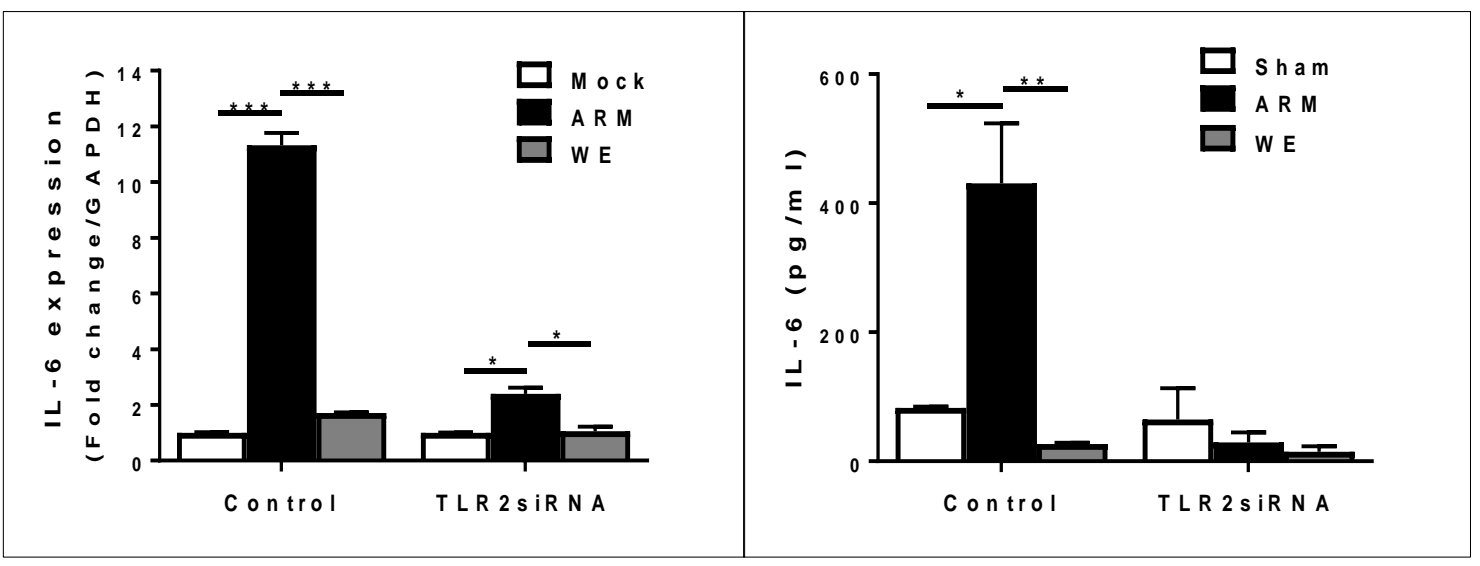

Figure 14. TLR2 siRNA transfection down regulates IL-6 in LCMV-ARMinfected murine macrophages.

Murine-specific TLR-2 siRNA, and Negative Control siRNAs (Ambion) were transfected into RAW264.7 cells using Lipofectamine (Lipofectamine RNAi Max Reagent, Invitrogen) for 48 hours. Transfected cells were infected either with LCMV-ARM or with LCMV-WE at MOI >1 PFU/cell incubated for 24 hours. A. IL6 mRNA expression was determined by real-time PCR. B. Protein expression of IL-6 was measured with a mouse IL-6 ELISA kit. Data (triplicate/group) represented as mean $\pm \mathrm{SEM},{ }^{*} p<0.05,{ }^{* *} p<0.01,{ }^{* * *} p<0.001$. All experiments done by Min Wang, Ph.D. 
siRNA resulted in moderate suppression of TLR-2. However, the TLR-2 silencing dramatically down-regulated IL-6 mRNA expression, and production of IL-6 protein in LCMV-ARM-infected cells. As expected, the effect of TLR-2 silencing on IL-6 in LCMV-WE-infected cells was minimal if any (Fig. 14).

\section{Co-staining experiments revealed an LCMV strain-specific pattern of} interactions between viral antigen and endosomal markers. TLRs sense the foreign invasion of microbes/viruses by recognizing their structural components and activate intracellular signaling pathways [219]. The TLR family includes receptors residing both on the cell surface, and in intracellular compartments. Early and late endosomes have unique TLR profile, and signaling properties [220, 221]. After internalization, virions are sorted into endocytic vesicles and delivered to endosomal/lysosomal compartments via the endocytic, and/or micropinocytosis pathways. LCMV and LASV enter cells through an unusual clathrin-, caveolin-, and dynamin-independent endocytic pathway [34, 35, 43, 44, 190, 218]. To assess the interaction between virus-loaded vesicles, and trafficking through the intracellular TLR-containing compartments, mock- and LCMV-infected cells were stained with monoclonal against the conserved LCMV NP epitope, and with markers of early and late endosomes. Intracellular colocalization of LCMV antigen and endosomal markers was visualized using immunofluorescence assay and confocal microscopy.

In the case of early endosomes, strong evidence of co-staining between the virus (NP antigen, green), early endosome antigen 1 (EEA1, red) and TLR-2 (light blue) was documented in LCMV-ARM-infected cells. Co-localized signals 


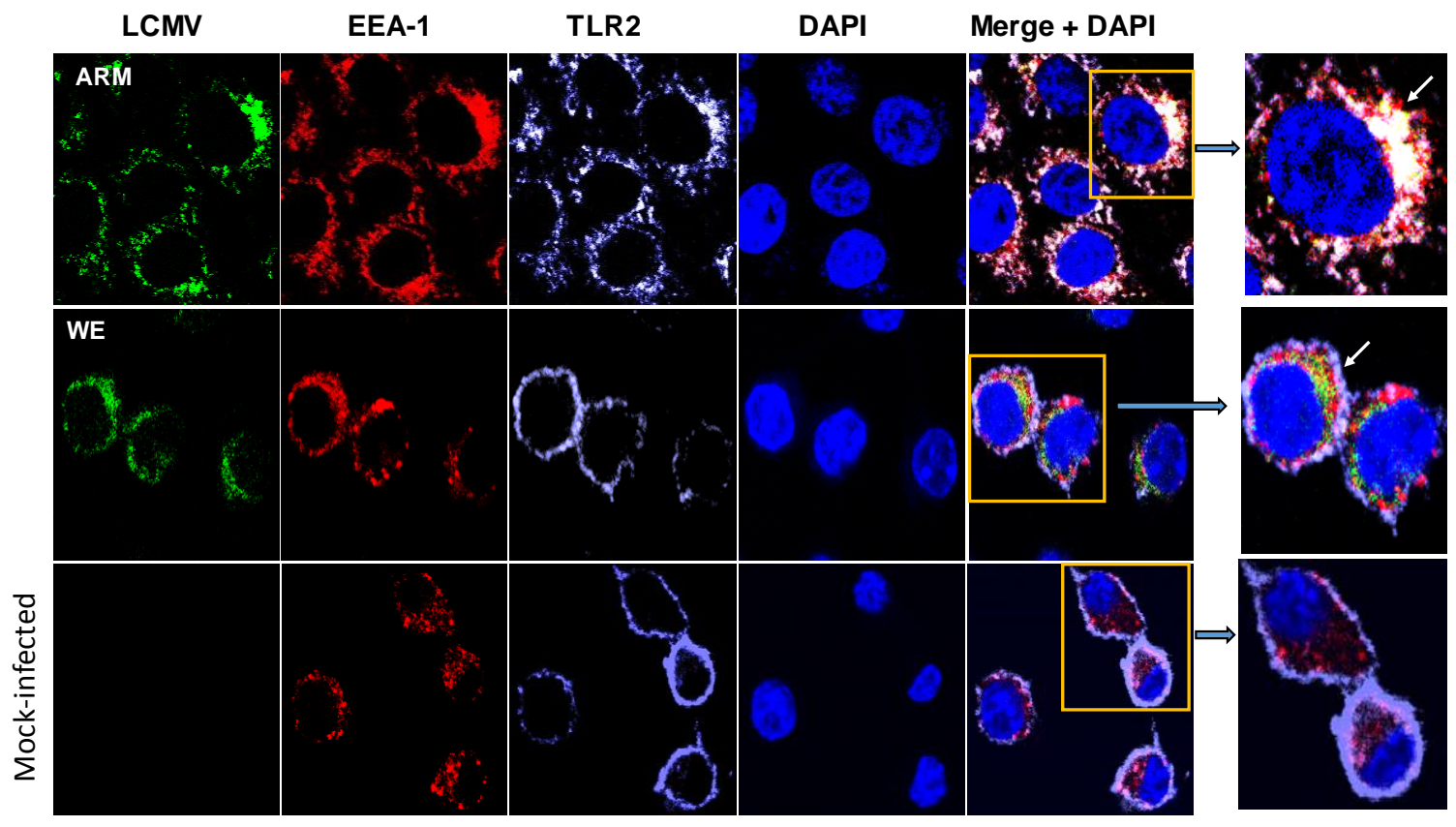

Figure 15. LCMV-ARM has strong co-localization patterns of LCMV NP with EEA-1 and TLR-2 markers.

RAW cells were infected with LCMV at MOI 2 PFU/cell and incubated for $30 \mathrm{~min}$.

Mock- and LCMV-infected cells were fixed, permeabilized and co-stained with monoclonal M104 (Abcam) against conservative NP epitope (green), EEA1 (monoclonal F.43.1, Thermo scientific, red), and TLR2 (monoclonal CD282, eBioscience, light blue). Upper panels, LCMV-ARM-infected cells, right panel indicate a cell co-stained with all three antibodies. Extensive white area of colocalized antigens indicated by arrow. Middle panels, LCMV-WE-infected cells, right panel, arrow indicates distinct staining pattern for individual antigens, no colocalization. Low panels, mock-infected cells. All experiments done by Min Wang, Ph.D. 
were seen as extensive white areas in the cytoplasm (Fig. 15). In contrast, all three colored markers had distinct staining patterns in LCMV-WE-infected cells with no evidence of co-staining. The small molecular weight G-protein, RAB-7, regulates late endocytic trafficking. Co-staining of LCMV-infected cells with antiNP and RAB-7 antibodies provided evidence of co-localization of target antigens in cells infected with both strains of LCMV, ARM and WE. Nevertheless, the costaining patterns were different between the two strains of virus, with evidence of more extensive bright-yellow areas of co-localized signals in LCMV-ARMinfected cells (about $80 \%$ ) in comparison with WE-infected cells (about 50\%; p<0.05) (Fig.16).

Fusion of LASV and LCMV glycoproteins with the cell membrane is triggered at very low $\mathrm{pH}(3.0-4.5)$ suggesting that these viruses can fuse not only in late endosomes, but also in the lysosomal compartment [218]. A unique feature of LASV infection is the usage of the second receptor, lysosomeassociated membrane protein-1 (LAMP-1, CD107), to trigger low-pH- and GPCmediated fusion with the late endosome membrane $[28,29]$. Using co-staining with anti-LAMP1 antibody, we have found colocalization between LCMV NP and LAMP-1 and; in line with previous observations, this assessment was LCMVstrain specific (Fig. 17). Co-localization of both signals, LCMV NP antigen (green) and LAMP-1 (red), was clearly seen as strong yellow spots in about $80 \%$ of LCMV-ARM-infected cells (Fig. 17, upper right panel). While small yellow areas can be found in about $50 \%$ of LCMV-WE-infected cells as well, the co-staining pattern was statistically less prominent ( $p<0.001$, Fig. 17$)$. 

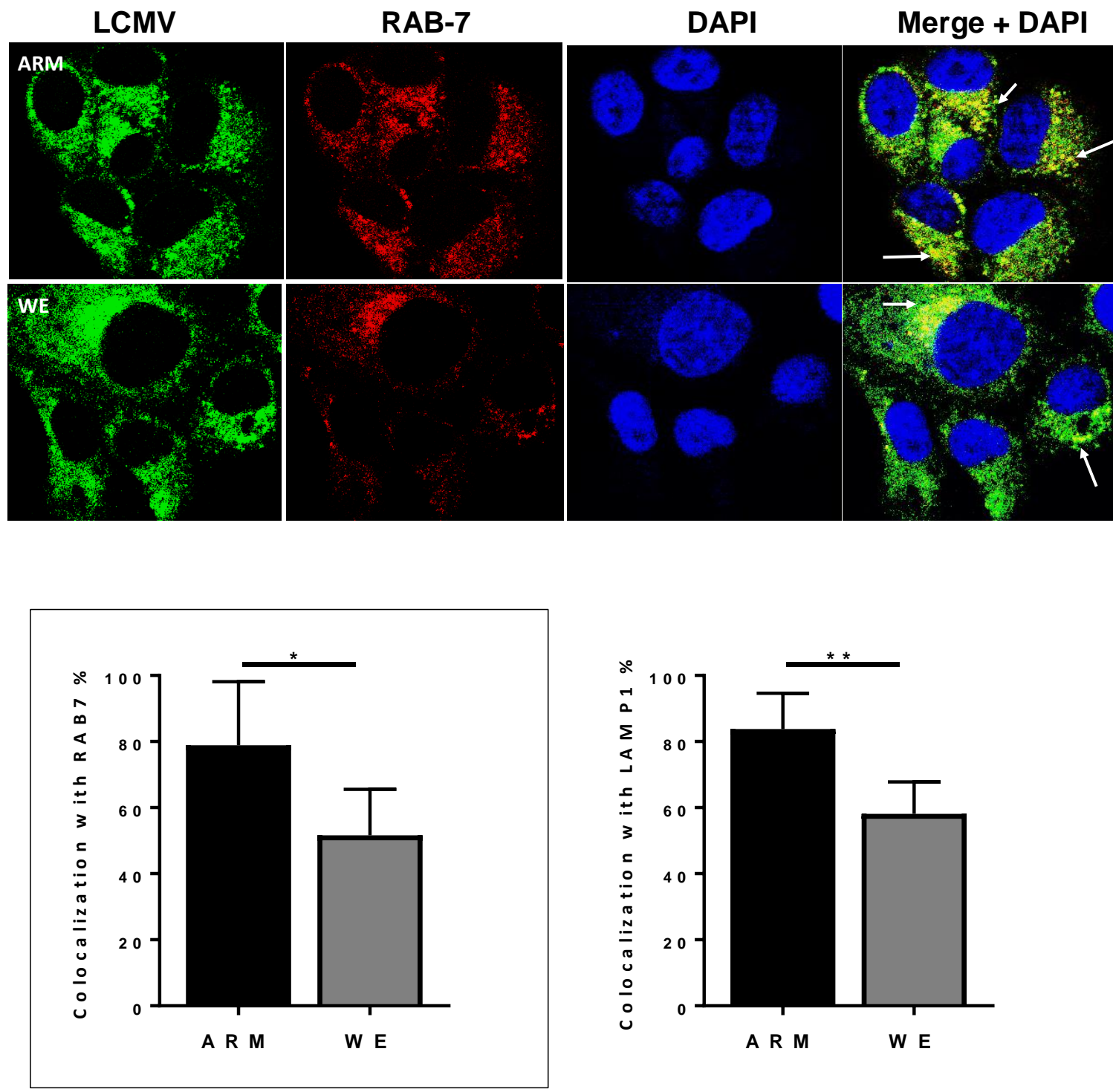

Figure 16. LCMV-ARM shows more co-localization with RAB-7 marker, compared to LCMV-WE.

Cells were infected with LCMV as described in legend to Fig. 11, incubated for 2 hrs, and co-stained with M104 and RAB-7 antibodies. Upper panels, ARMinfected cells, white arrows indicate bright yellow co-localized spots. Low panels, WE-infected cells, white arrows indicate diffuse light-yellow areas of colocalization. See also Fig. 14. The analysis was performed as recently described for LCMV infected cells using ZEN software [214] with slight modifications. Up-to 
eight LCMV NP-positive (green cells) cells were marked and compared pixel by pixel with intensity signals from each channel. Background for green and red channels was determined based on mock-infected cells staining. Total (greenand red-positive) signals were divided by the sum of green positive pixels and expressed as co-localization efficiency, (\%). The calculation was repeated for individual cells. All experiments done by Min Wang, Ph.D. 

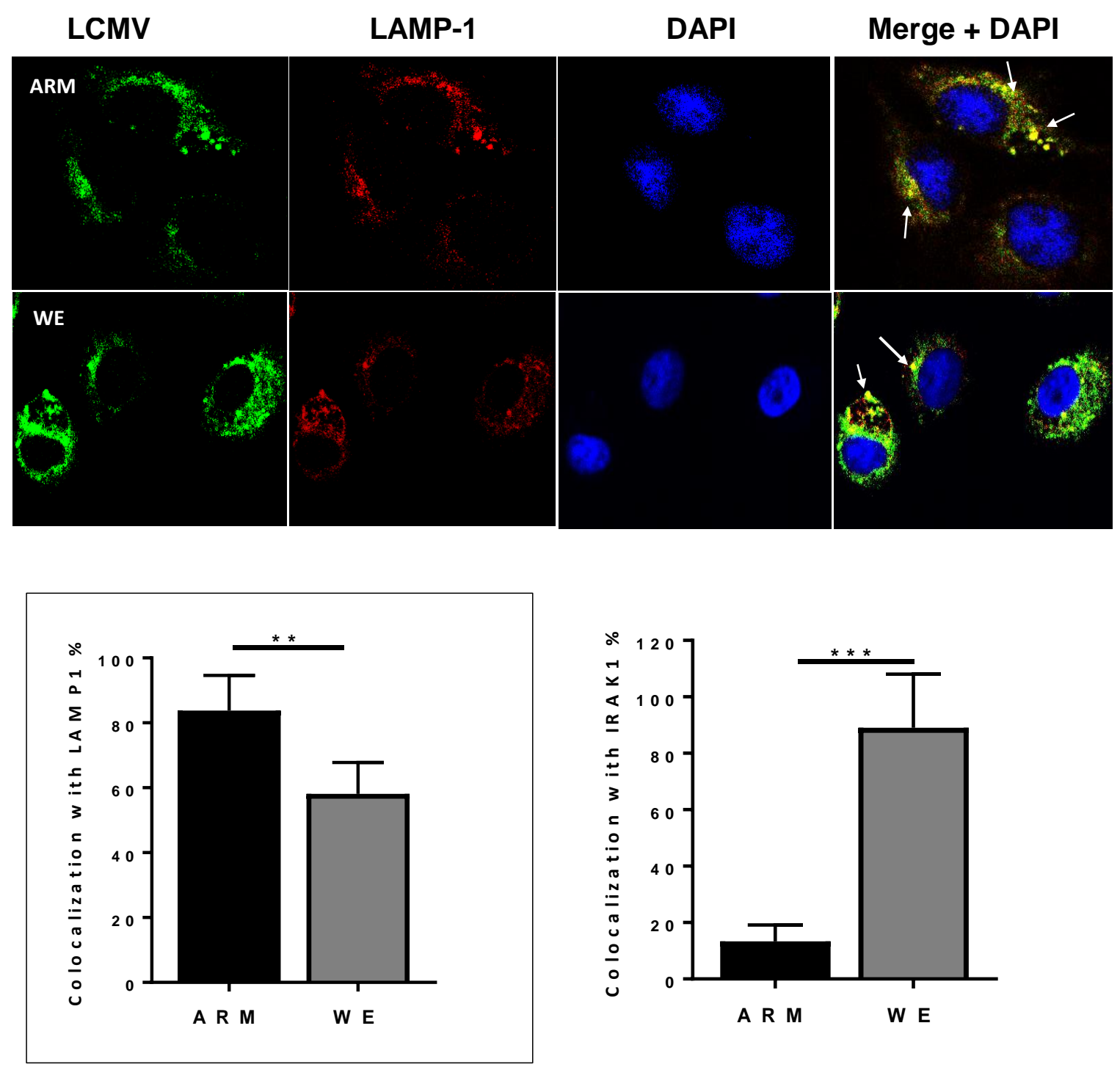

Figure 17. LCMV-ARM has a stronger co-staining pattern with LAMP-1 than LCMV-WE.

Cells were infected and treated as indicated in legend to Fig. 12. Upper panels, ARM-infected cells. Lower panels, WE-infected cells. Co-staining areas are indicated by white arrows. See also Fig.14. The analysis was performed as recently described for LCMV infected cells using ZEN software with slight modifications [214]. Up-to eight LCMV NP-positive (green cells) cells were marked and compared pixel by pixel with intensity signals from each channel. 
Background for green and red channels was determined based on mock-infected cells staining. Total (green-and red-positive) signals were divided by the sum of green positive pixels and expressed as co-localization efficiency, (\%). The calculation was repeated for individual cells. All experiments done by Dr. Min Wang. 
Interaction with IRAK-1, mediator of TLR-induced signaling, is different in LCMV-ARM- versus LCMV-WE-infected cells. TLR signaling is dependent on the recruitment of several key adaptor molecules. Upon ligand recognition, MyD88 (and/or Mal) recruits and activates IL-1-associated kinases (IRAK), such as IRAK-1, triggering a down-stream activation cascade leading to NF-kB translocation and transcription initiation [222]. Notably, in cells transfected with an NF-kB-luciferase reporter, infection with LCMV-ARM resulted in the induction of NF-KB, but cells infected with LCMV-WE and immunosuppressive LCMV Clone 13 did not [167].

In the next experiments, co-staining with anti-IRAK-1 antibody was performed in LCMV-infected murine macrophages. As seen in Fig. 18 and Fig. 19, little evidence of interaction between LCMV NP and IRAK-1 was found in LCMV-ARM-infected cells, with only $15 \%$ of the cells co-staining for LCMV NP and IRAK-1. In contrast, co-localized signals, identified as extensive yellow areas, reached about $87 \%$ in LCMV-WE-infected cells.

In response to stimulation, IRAK-1 is subjected to ubiquitination and degradation, and IRAK-1 protein level remained suppressed up to $8 \mathrm{~h}$ after stimulation [223]. To track LCMV strain-specific differences in IRAK-1 expression in infected cells, Western blot analysis was performed to detect IRAK-1 in infected cells (Fig. 19). While there was no difference between expressions of IRAK-1 at 24h after infection in LCMV-ARM and WE-infected cells, the IRAK-1 expression levels were higher at later time points in LCMV-WE-infected cells in comparison with mock- and ARM-infected cells (Fig. 19). 


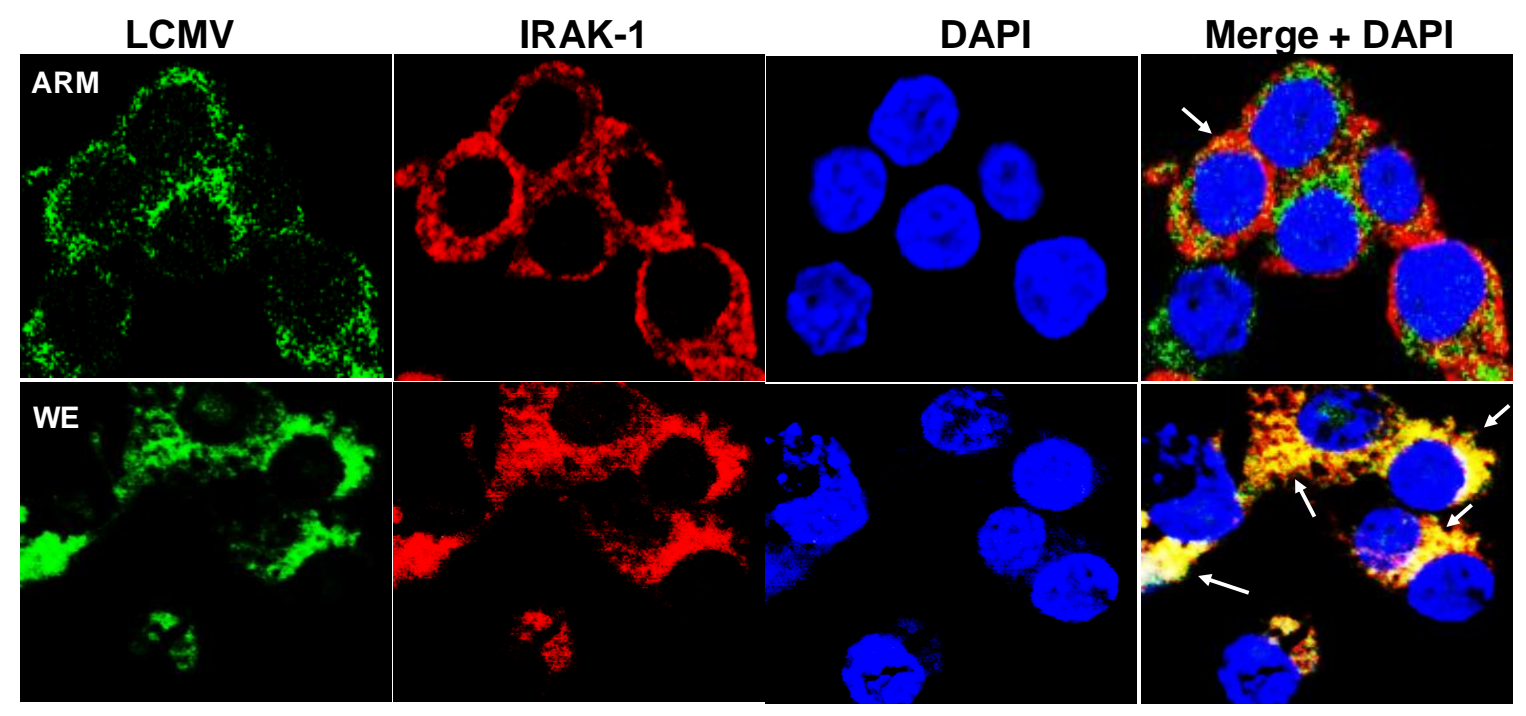

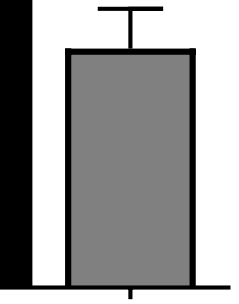

W E

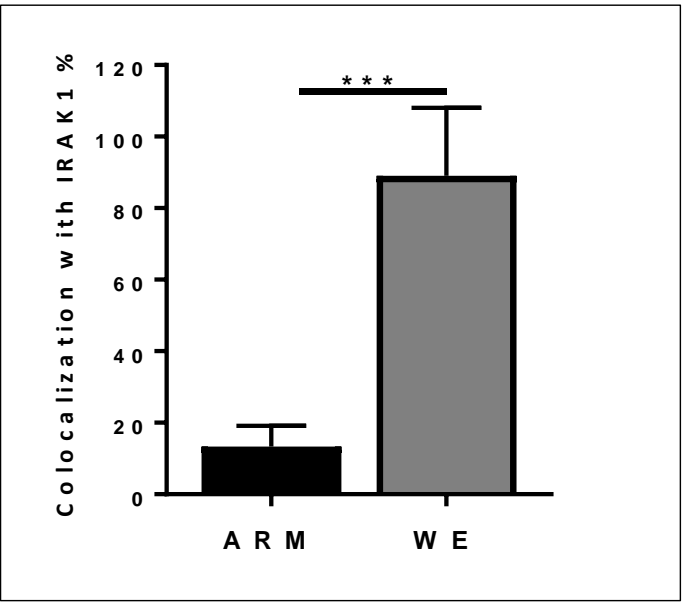

Figure 178. LCMV-WE has significant co-staining patterns with IRAK-1 compared to LCMV-ARM.

Cells were infected with LCMV strains and incubated for $1 \mathrm{hr}$. Upper panels, ARM-infected cells. Low panels, WE-infected cells. Extensive yellow areas of co-localized staining in WE-infected cells are shown by arrows. The analysis was performed as recently described for LCMV infected cells using ZEN software with slight modifications. Up-to eight LCMV NP-positive (green cells) cells were marked and compared pixel by pixel with intensity signals from each channel 
[214]. Background for green and red channels was determined based on mockinfected cells staining. Total (green-and red-positive) signals were divided by the sum of green positive pixels and expressed as co-localization efficiency, (\%). The calculation was repeated for individual cells. All experiments done by Min Wang, Ph.D. 


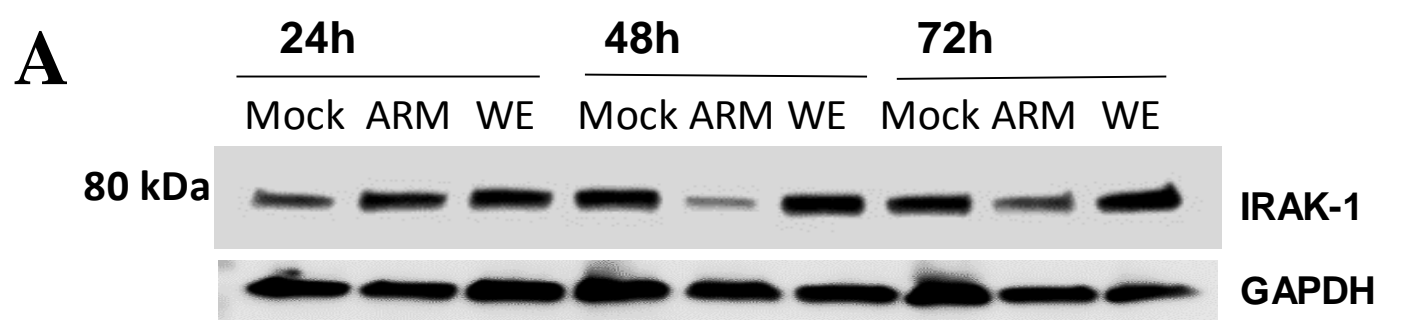

B

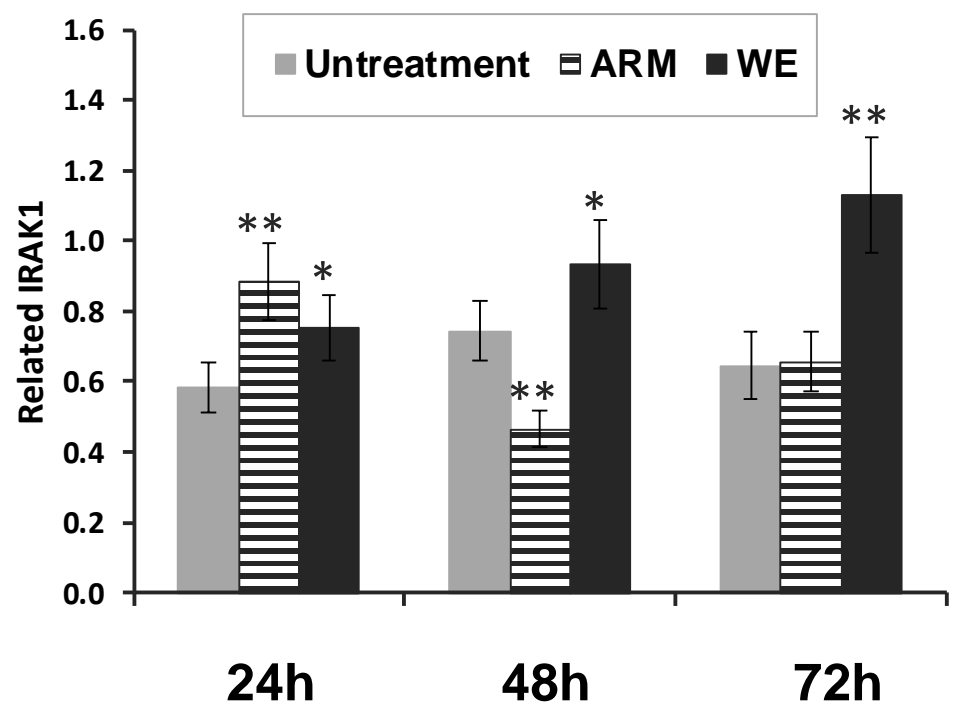

${ }^{*} \mathrm{p}<0.05 ; * * \mathrm{p}<0.01$ in comparison with mock-infected cells

Figure 19. Expression of IRAK-1 in LCMV-infected cells.

RAW267.7 cells were grown in T25 flasks and infected with LCMV viruses an MOI 0.1. At different time post infection, protein extracts were prepared and subjected to SDS-PAGE analysis as previously described [224]. In brief, protein samples were combined with 4 X Laemmli sample buffer and loaded onto SDSpolyacrylamide gels of $10 \%$ and $15 \%(\mathrm{w} / \mathrm{v}$ ) acrylamide followed by electrophoresis and Western blotting onto PVDF membranes. Primary antibodies against IRAK-1 (D51G7, Cell Signaling) and GAPDH (sc-25778, Santa Cruz Biotechnology) were used at dilutions 1:1000 and 1:2000, respectively. Bands were visualized using horseradish peroxidase-coupled secondary antibodies, an 
ECL kit (Pierce, Rockford, IL) and Hyperfilm (GE Healthcare, Piscataway, NJ). A.

IRAK-1 was identified as a $80 \mathrm{kDa}$ band. B. Densitometric analysis was

performed using UN-SCAN-IT gel (Silk Scientific Inc., Orem, UT) software. All experiments done by Dr. Min Wang, Ph.D. 


\section{Probing LCMV-ARM versus LCMV-WE infection with inhibitor drugs.}

Antivirals with known mechanisms of action are a useful tool to study virus-cell interaction and cell factors involvement in virus replication. In this study, representatives of three groups of drugs targeting host factors at the cell surface, microtubules, and cell membrane fusion in late endosomes, were used to validate results presented in the previous sub-sections.

It was documented that entry of LCMV (Clone 13) was dependent upon membrane cholesterol [36]. To address LCMV-strain specific sensitivity to cholesterol depletion, Vero cells were initially used to reproduce previously published results [35]. The cells were pretreated with $5 \mathrm{mM}$ of $\mathrm{M} \beta-\mathrm{CD}$, infected with LCMV at an MOI of $0.3 \mathrm{PFU} /$ cell, and incubated with or without drug during 24h after infection, as described in Materials and Methods. As expected, replication of both strains of LCMV was affected by depletion of cholesterol on the cell surface (Fig. 20 A). However, when the LCMV infectious yields were normalized to untreated cells and compared to each other, LCMV-WE infection was more sensitive to the cholesterol depletion (at least 2-fold differences, Fig. $20 \mathrm{C}$ ), to $M \beta-C D$ in comparison with LCMV-ARM infection. The LCMV-strain specific sensitivity to cholesterol depletion was consistently observed Caco-2 cells, independent of polarization status of these cells (Fig. 21 and Fig. 22).

A previously published study documented that disruption of microtubules with nocodazole inhibited replication of LCMV-ARM [35]. In line with these results, replication of both strains of LCMV demonstrated almost equal sensitivity 

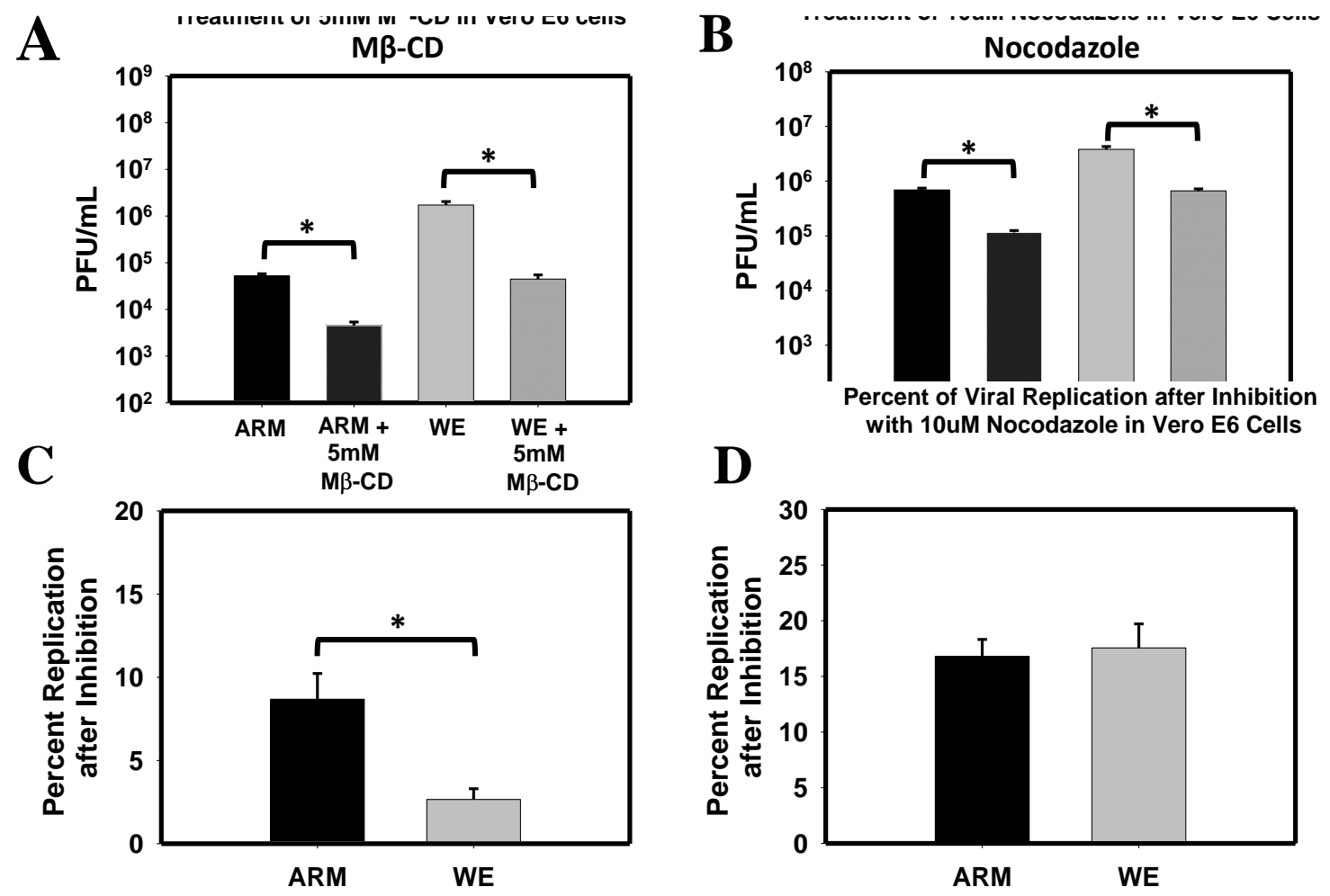

Figure 20. Depletion of membrane cholesterol and microtubules inhibits LCMV-Armstrong and LCMV-WE replication in VeroE6 cells.

Vero E6 were treated for 1 hour prior to infection with $5 \mathrm{mM} \mathrm{M \beta -CD} \mathrm{(A} \mathrm{and} \mathbf{C})$ or $10 \mu \mathrm{M}$ nocodazole (B and D), then infected with LCMV as described in Materials and Methods. Supernatants were collected $24 \mathrm{hrs}$ after infection and virus production was determined by plaque assay (A and $\mathbf{B})$. Values are shown as the mean of 3 biological replicates with the error bar representing the standard deviation. Percent replication after inhibition was normalized to untreated PFU/mL (C and D). Standard T-test was used to analyze differences in titer and percentages. ${ }^{*} p \leq 0.05$ 


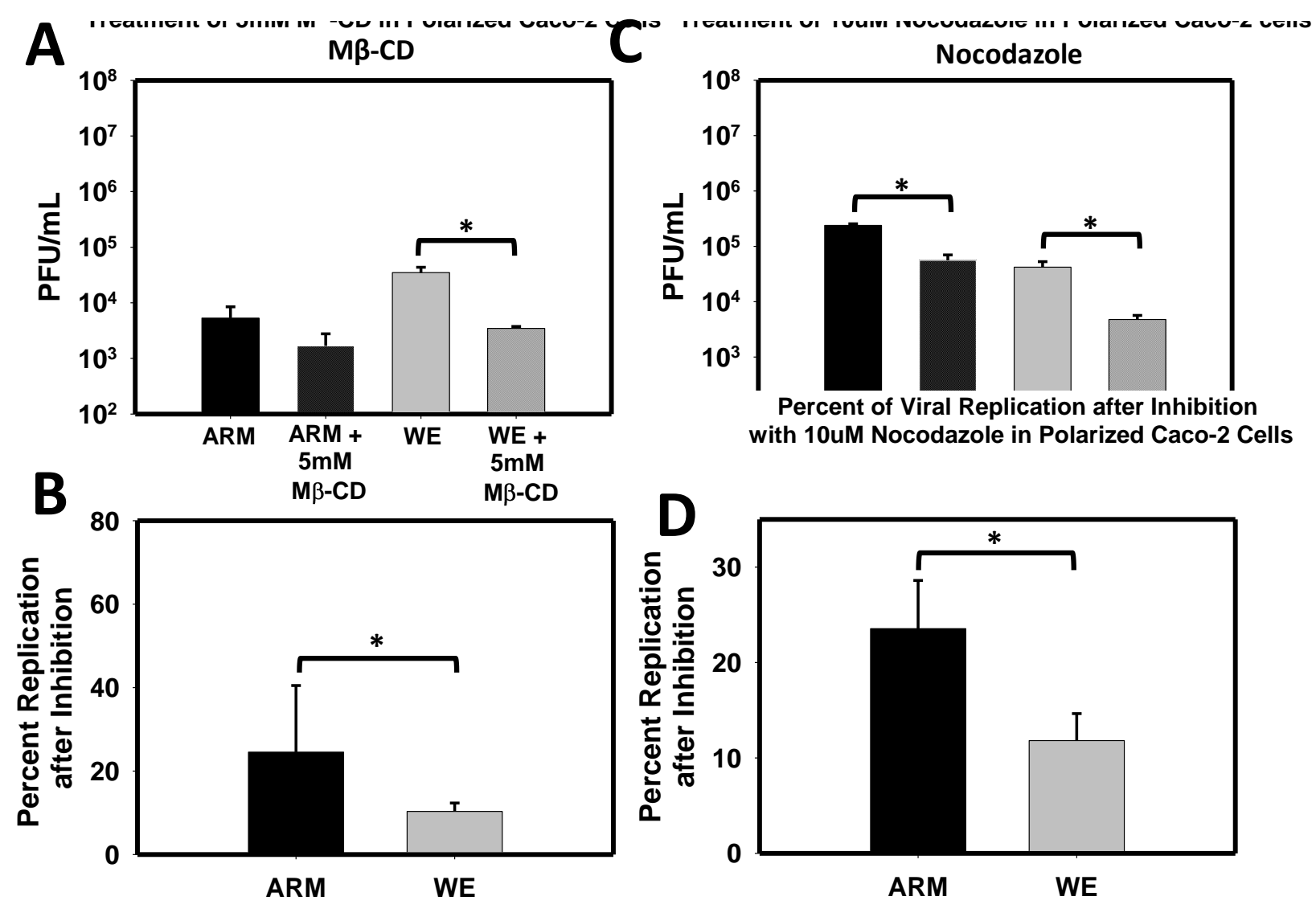

Figure 21. Depletion of membrane cholesterol and microtubules inhibits LCMV-Armstrong and LCMV-WE replication in polarized Caco-2 cells.

Caco-2 cells were seeded in 12-well plates and allowed to polarize for 3 weeks. Cells were treated for 1 hour prior to infection with $5 \mathrm{mM} \mathrm{M \beta -CD} \mathrm{(A} \mathrm{and} C)$ or $10 \mu \mathrm{M}$ nocodazole (B and D), then infected with LCMV-ARM (black) or LCMV-WE (grey) at a multiplicity of infection (MOI) of $0.3 \mathrm{PFU} /$ cell for 1 hour on ice. After infection, maintenance media was added to cells in the absence (solid) or presence (striped) of $5 \mathrm{mM} \mathrm{M \beta -CD} \mathrm{(A)} \mathrm{or} 10 \mu \mathrm{M}$ nocodazole (C). Supernatants were collected 24hours after infection and virus production was determined via standard plaque assay ( $\mathrm{A}$ and $\mathrm{C}$ ). Values are shown as the mean of 3 biological replicates with the error bar representing the standard deviation. Percent 
replication after inhibition was normalized to untreated $P F U / m L$ (B and $D$ ).

Standard T-test was used to analyze differences in titer and percentages. ${ }^{*} \mathrm{p} \leq 0.05$ 

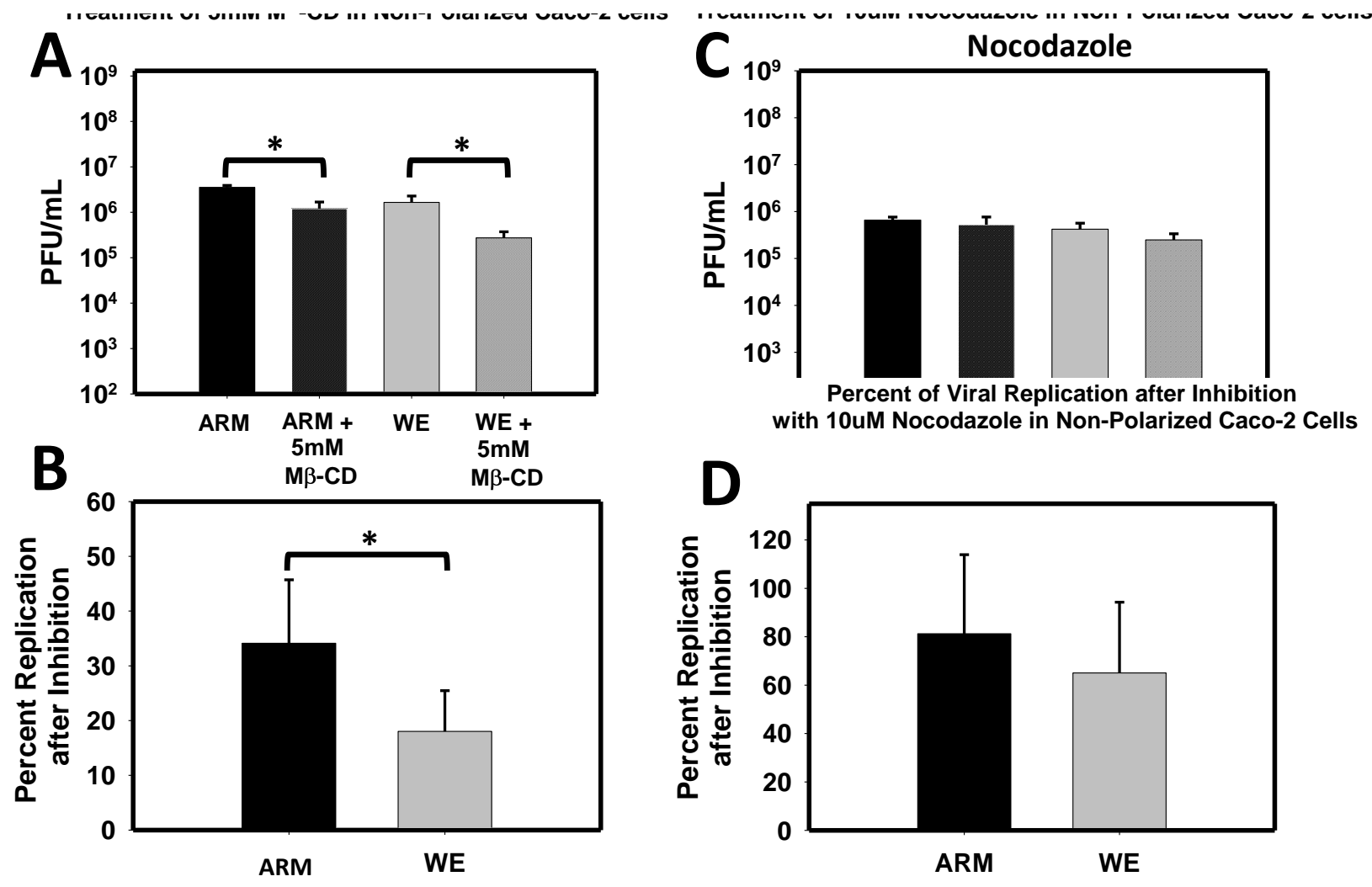

Figure 182. LCMV-ARM and LCMV-WE replication is inhibited by depletion of membrane cholesterol, but not by microtubule disruption in nonpolarized Caco-2 cells.

Caco-2 cells were seeded in 12-well plates. Cells were treated for 1 hour prior to

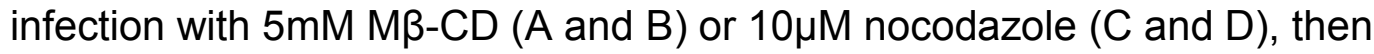
infected with LCMV-ARM (black) or LCMV-WE (grey) at a multiplicity of infection (MOI) of $0.3 \mathrm{PFU} / \mathrm{cell}$ for 1 hour on ice. After infection, maintenance media was added to cells in the absence (solid) or presence (striped) of $5 \mathrm{mM} \mathrm{M} \beta-C D(A)$ or $10 \mu \mathrm{M}$ nocodazole $(\mathrm{B})$. Supernatants were collected 24 hours after infection and virus production was determined via standard plaque assay (A and C). Values 
are shown as the mean of 3 biological replicates with the error bar representing the standard deviation. Percent replication after inhibition was normalized to untreated PFU/mL (B and D). Standard T-test was used to analyze differences in titer and percentages. ${ }^{*} p \leq 0.05$ 
to the drug (Fig. $20 \mathrm{~B}$ and D). Treatment of infected Caco-2 cells with nocodazole generated controversial results. In polarized cells, replication of both strains of LCMV was sensitive to disruption of microtubules, with LCMV-WE infection being more sensitive to the treatment (Fig. $21 \mathrm{~B}$ and D). Surprisingly, LCMV infection in non-polarized Caco-2 cells was not sensitive to nocodazole treatment (Fig. 22 B and $D$ ). The fusion of arenavirus proteins with cell-derived membranes is the last step of intracellular trafficking of the virus-containing vesicles. The low $\mathrm{pH}-$ mediated fusion occurs in late endosomes/lysosomes and can be blocked by drugs raising $\mathrm{pH}$ in this sub-cellular compartment [225]. To assess LCMV strainspecific sensitivity to inhibitors blocking fusion with cell membrane, an established protocol was used to treat Vero cells with bafilomycin A1 to prevent acidification of the late endosomes [218].

As expected, bafilomycin treatment inhibited replication of both strains of LCMV in a dose-dependent manner as assessed by plaque assay and quantitation of viral RNA load by PCR (Fig. 24). When compared with mocktreated cells, the differences ranging from 1-2 and 2-3 logs PFU/ml were observed in LCMV-WE- and ARM-infected cells, respectively (Fig. 23 A). When normalized to viral input, a significant difference of about $8 \%$ was observed between LCMV-ARM- and -WE-infected cells treated with $100 \mathrm{nM}$ of bafilomycin (Fig. 24). Similarly, when viral RNA was measured and normalized to mocktreated cells, a 2-fold difference was observed between LCMV-ARM- and -WEinfected cells, confirming higher sensitivity of LCMV-ARM infection to $\mathrm{pH}$ changes in late endosomes in comparison with LCMV-WE (Fig. 23 B). 
A

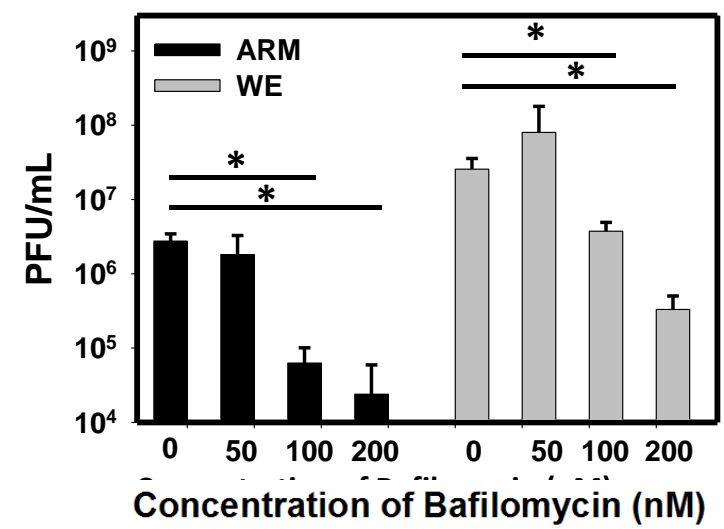

Vero E6 cells Treated with Bafilomycin

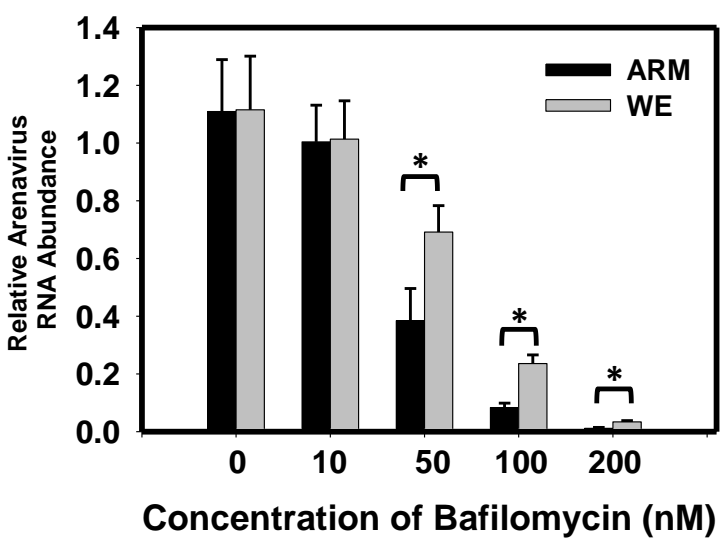

Figure 23. LCMV strain-specific replication sensitivity to $\mathrm{pH}$ increases in late endosomes.

VeroE6 cells were treated with bafilomycin A1 and infected with LCMV as described in Materials and Methods. Supernatants and cells were collected 24 hrs after infection and virus production was determined via standard plaque assay (A) and viral mRNA was determined using qRT-PCR and normalized to untreated cells (B). Standard T-test was used to analyze differences in titer and percentages. * $p \leq 0.05$ 


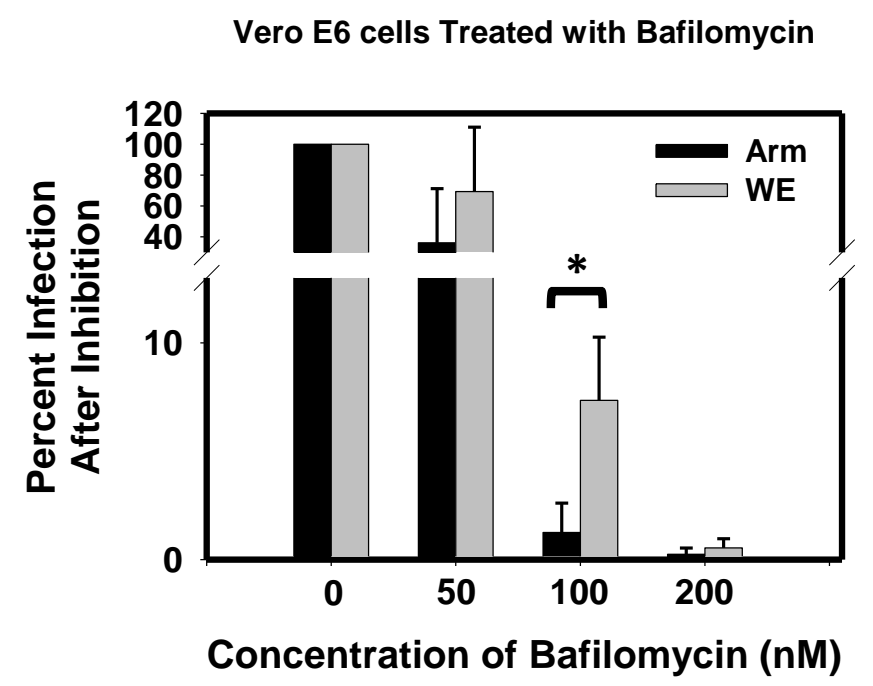

Figure 194. Blocking of late endosome acidification results in a decrease in the percentage of viral infection in both LCMV-ARM and LCMV-WE in a strain-specific manner.

VeroE6 cells were seeded in 12-well plates. Cells were treated for 1 hour prior to infection with varying concentrations of bafilomycin. Cells were then then infected with LCMV-ARM (black) or LCMV-WE (grey) at a multiplicity of infection (MOI) of 1.0 PFU/cell for 1 hour on ice. After infection, maintenance media was added to cells in the absence $(0 \mathrm{mM})$ or presence $(50-200 \mathrm{nM})$ of bafilomycin. Supernatants were collected 24 hours after infection and virus production was determined via standard plaque assay. Percent infection was determined dividing PFU/mL by OmM PFU values. Data are shown as the mean of 3 biological replicates with the error bar representing the standard deviation. Percent replication after inhibition was normalized to untreated PFU/mL ( $B$ and D). Standard T-test was used to analyze differences in titer and percentages. * $\mathrm{p} \leq 0.05$ 


\section{DISCUSSION}

With a high genetic and biological diversity among LCMV strains, the behavior of WE and ARM strains of LCMV resembles phenotypic features of LASV and MOPV in animal models. Indeed, similar to LASV, LCMV-WE induces fatal disease in guinea pigs and fatal LF-like hepatitis in Rhesus macaques [194, 195]. In both animal models, reassortant analysis revealed that the L RNA segment of LASV and LCMV-WE is the major genetic factor responsible for fatal outcome [204, 205]. Similarly, in LCMV-induced hepatitis in mice, the L polymerase of WE strain was primarily responsible for liver pathogenicity, with minimal contribution of the GPC gene [226]. Notably, a single-point K1079Q mutation in the L polymerase of LCMV-ARM was necessary and sufficient, in part, to transform LCMV-ARM into immunosuppressive Clone 13. The F260L mutation in GP1, which was associated with increased affinity to $\alpha-D G$ and targeting dendritic cells, played an accessory role in driving the duration of persistence and generalization of immunosuppression in mice [227]. Taken together, these results provide strong justification to consider LCMV-WE as a surrogate model of LASV, causing fatal hepatitis in NHPs [195].

Dendritic cells and macrophages are primary targets for LASV and LCMVWE. Dysregulation of myeloid cells, immunosuppressive features of LASV infection, and failure to induce sustainable innate and adaptive immunity, resulted in fatal LF outcome $[206,207]$. In contrast, DCs and macrophages are strongly activated by MOPV, and the infection upregulates co-stimulatory molecules, IFN type I, and pro-inflammatory cytokines [106]. 
Strong activation of monocyte-macrophages seems to be a general feature of non-pathogenic mammalian arenaviruses [102, 106, 158-162]. We have previously demonstrated that MOPV and LCMV-ARM induced proinflammatory cytokines, and this induction required virus replication and was dependent on TLR2/CD14/Mal/NF-KB signaling [167]. In addition to LCMV-ARM and MOPV, TCRV (but not pathogenic JUNV) induced cytokines release from macrophages as well [158]. With these similarities, TLR2/Mal/MyD88-dependent signaling has some differences between the OW and NW mammalian arenaviruses. In the case of Candid 1 (attenuated JUNV vaccine), engagement of TLR2/6 heterodimers on cell surface by viral glycoprotein was sufficient to trigger cytokine response via RIG-I/MDA5, NF-KB, and Erk1/2 pathways and did not require internalization and viral replication.

In this study, we provide additional evidence of TLR-2 involvement in the induction of pro-inflammatory cytokines by LCMV-ARM (not LCMV-WE), since TLR-2 silencing resulted in abrogation of the response to LCMV-ARM. Accumulated evidence demonstrates a role for TLR-2 in sensing mammalian arenaviruses with different pathogenic potential [159-161, 167, 228, 229]. Viral glycoprotein are recognized as a TLR-2 ligand for JUNV (wild type and vaccine strain) [229], however, a TLR-2 ligand has not been identified for the OW arenaviruses. Viral glycoproteins of the OW mammalian arenaviruses can be considered as TLR-2 ligands similar to JUNV. However, in contrast to JUNV, TLR2/CD14/Mal-dependent signaling induced by MOPV and LCMV-ARM 
required virus replication [167], indicating a more complex nature of virusinduced innate responses.

In addition to TLR-2, LCMV and LASV induce TLR-7 and TLR-9 responses and activate RIG-I [230]. These major machineries initiate intracellular signaling via common cytoplasmic adapters (e,g., MyD88/Mal, TRIF, IPS-1) to induce IFN type I and NF-kB-dependent pro-inflammatory cytokines. It seems intracellular TLRs involved in arenavirus recognition have different intracellular localization [220, 221]; TLR-2 has been mostly associated with early endosomes (Fig. 2), while TLR-7 and TLR-9 have been sitting predominantly in late endosomal compartment since treatment with bafilomycin A1 and chloroquine inhibits the activation of nucleic acid sensing TLRs [221].

Pathogenic viruses have developed tricky mechanisms to subvert endocytic and pathogen-sensing functions of DCs and macrophages to avoid effective recognition and immune control [231]. As seen in Fig. 15-17, ARM and WE strains of LCMV had different patterns of intracellular trafficking and interaction with endosomal compartments. Non-pathogenic (for NHPs) LCMVARM was tightly interacted with early and late endosomes, as it was revealed by co-staining with EEA-1, RAB-7 and LAMP-1 markers. Alternatively, LCMV-WE, causing fatal LF-like hepatitis in NHPs, effectively avoid early endosome recognition. Consistent with this, penetration kinetics study documented that LCMV-WE reached late endosomes by bypassing RBA5/EEA1-positive compartment [44]. Notably, in late endosomal/lysosomes, LCMV-WE infection demonstrated less extensive interaction with RAB-7 and LAMP-1 markers. 
After interaction with cell surface receptors, LASV switches to a second receptor, LAMP-1, to fuse with cell membrane in late endosomes/lysosomes [28]. While recent studies provided evidence that LAMP-1 is not absolutely required for LASV infection [50], LAMP-1 increases efficiency of infection by promoting fusion in less acidic endosomal environment. Interestingly, LAMP-1 binding site interaction with LASV GP1 has not been shown to be shared by other OW arenaviruses, and was not found in MOPV and LCMV [49]. It seems the LAMP-1 positive co-staining pattern observed in LCMV-infected cells (Fig. 4) does not relate to LCMV GP1 and is probably linked to glycoprotein responsible for fusion, GP2.

To elicit MyD88/Mal-dependent pro-inflammatory responses, IRAK-4 is recruited to phosphorylate IRAK-1 and IRAK-2, to further activate down-stream cascade via the E3 ubiquitin ligase TRAF-6 and TAK-1 phosphorylation of IKKB, resulting in IKB degradation and release of NF-KB [220]. Interestingly enough, LCMV-WE infection of murine macrophages resulted in strong co-localization of viral NP with IRAK-1 (Fig. 5). Taking into consideration suppression of NF-KB in LCMV-WE-infected cells [167], this co-staining pattern seems to contribute to the immunosuppressive phenotype of LCMV-WE, but preventing transcription of NF$\mathrm{KB}$ factors.

Epithelial cells of the gastrointestinal tract and upper-respiratory system are major gates of entry for LASV and LCMV. An epidemiological study in LASV endemic areas identified the hunting of peridomestic rodents and consumption of LASV-contaminated food, as risk factors for rodent-to-human transmission [137]. 
Similar to LASV, LCMV was resistant to low $\mathrm{pH}$ after oral (lavage) application. Notably, experiments in mice $[169,170,232]$, and NHPs $[155,156,182]$, suggest that crossing the gastric/intestinal mucosa resulted in virus attenuation and protection of animals against subsequent fatal challenge. Experiments in NHPs revealed clear differences in pathogenic potential between LCMV-ARM, causing deeply attenuated asymptomatic infection, and LCMV-WE, inducing LF-like fatal disease in all animals after systemic application. Notably, while in some animals, oral inoculation of LCMV-WE resulted in viremic manifested disease, animals recovered and became protected against fatal intravenous challenge [182].

The possible LCMV strain-specific interaction with gastrointestinal epithelia was tested in polarized Caco-2 cells, and both strains of LCMV, demonstrated similar attachment/binding profile in these cells [208]. In this study, Caco-2 cells were used to test well-characterized drugs affecting membrane cholesterol and disrupting microtubular transport. Previous studies documented that attachment of OW mammalian arenaviruses to cellular receptors occurs independently on cholesterol, while cholesterol depletion affected internalization and reduced replication indicating that efficient virus infection required membrane cholesterol $[35,138]$. In line with these results, treatment of Vero and Caco-2 cells (non-polarized and polarized) consistently demonstrated high sensitivity of LCMV-WE infection (vs. LCMV-ARM) to cholesterol depletion (Fig. 20, 21, and 22, $A$ and $C)$.

While the actin cytoskeleton is not required for LCMV pseudotypes and LCMV-ARM replication, disruption of microtubules with nocodazole affected 
replication of these viruses in CV1 cells [35]. Consistently, the drug had similar inhibitory effect on replication of both strains of LCMV in Vero cells. Interestingly enough, polarization status of Caco-2 also had effect on strain-specific sensitivity of LCMV to nocodazole. In polarized cells, LCMV-WE infection was more sensitive to disruption of microtubules (Fig. 21 B and D). In contrast, replication of both strains of LCMV in non-polarized cells was not affected by nocodazole (Fig. 22, B and D). It suggests that polarization itself promotes contribution of microtubules to virus replication and results in LCMV strain-specific sensitivity to the drug (Fig. $22 \mathrm{C}$ and $\mathrm{D}$ ).

The final step of the endocytic pathway is $\mathrm{pH}$-dependent fusion with cell membranes at late endosomes/lysosomes, and the release of viral RNA to initiate productive infection. Using lysosomotropic agents $\left(\mathrm{NH}_{4} \mathrm{Cl}\right.$, chloroquine, monensin) we [225], and others [213] documented that LASV infection was more resistant to $\mathrm{pH}$ increase in comparison to non-pathogenic MOPV. This is in line with findings that demonstrated a very low $\mathrm{pH}$ requirement for LASV fusion [218]. Similar to lysosomotropic compounds, bafilomycin A1, an inhibitor of proton pumps, prevents acidification of late endosomes. Consistently, bafilomycin A1 also inhibited LASV and LASV pseudoparticles entry in dose-dependent manner [218]. Bafilomycin treatment of LCMV-infected cells resulted in inhibition of LCMV replication as well (Fig. 7, S5). Importantly, we have found that LCMV-WE infection was more resistant to bafilomycin A1 than LCMV-ARM infection, in good collaboration with LASV vs. MOPV sensitivity to lysosomotropic compounds [213, 225]. Different sensitivity of pathogenic vs. non-pathogenic OW arenaviruses to 
$\mathrm{pH}$ increase in late endosomes/lysosomes can affect viral RNA release kinetics. Additionally, change in $\mathrm{pH}$ can affect activation pattern of nucleic acid sensing TLR-7, TLR-9 and RIG-I driving expression of IFNs and pro-inflammatory cytokines. These factors are crucially involved in activation of antigen presenting cells and T cells stimulation resulting in efficient adaptive responses to control viral infection in case on infection with non-pathogenic viruses. In contrast, the immunosuppressive phenotype of LASV and LCMV-WE in addition with intracellular trafficking avoiding exposure with TLRs and delayed TLR-7, TLR-9 and RIG-I activation contributed to adaptive immune failure. Strong interaction between LASV NP and IRAK-1 in LCMV-infected cells is consistent with ability of this infection down-regulate NF-KB activity in infected cells [167].

\section{CONCLUSIONS}

In summary, in this study we demonstrated clear differences in terms of intracellular trafficking and sensitivity to pharmacological drugs between two strains of LCMV with different pathogenic potential for NHPs, WE and ARM. Consistent with previous results, LCMV-ARM co-staining pattern and interaction with endosomal compartments hosting TLRs, promotes induction of strong innate responses via TLR2/CD14/Mal-dependent signaling pathways. In addition, higher sensitivity of LCMV-ARM infection to bafilomycin A1 treatment suggests efficient release of viral RNA to activate nucleic acid sensing TLRs and RIG-I, contributing to development of robust adaptive immune response. 
CHAPTER 4

CONCLUSIONS AND FUTURE DIRECTIONS 


\section{CONCLUSIONS}

The overall objective of this research was to identify an intestinal epithelial cell culture model that could further lead us to understand how OW arenaviruses were interacting at the sight of intragastric infection, as well as investigate potential factors that drive pathogenic differences between viruses sharing similar genetic phenotypes. In order to study these differences, we utilized a welldeveloped intestinal epithelial model, polarized Caco-2 cells, to identify characteristics of infection with different $\mathrm{OW}$ arenaviruses. In addition, we used chemical inhibitors to identify differences in intracellular pathways of these closely related viruses.

In some villages in LASV endemic regions in West Africa up to $45 \%$ of the populations is seropositive for the virus, allowing for clearance of the disease without manifestation upon reinfection [81]. However, what defines why some individuals are seropositive and can protect themselves from disease onset, while others show clinical manifestation of LF, has yet to be defined. Our first goal was to develop and characterize a cell culture system as an in vitro model to investigate the interaction of OW mammalian arenaviruses at the sight of intragastric inoculation.

Caco-2 cell polarization results in development of tight junction proteins, monolayers of connected cells, and organizational patterns that differ from nonpolarized cell types [173]. The first step was characterizing how different OW arenaviruses interact at the site of intragastric inoculation. Due to the difference 
in phenotypes of polarized and non-polarized cells, we analyzed this in the context of viral replication in Caco-2 cells. We found that the polarity of this cell line did not impact the replication kinetics of LCMV, MOPV, or ML-29.

In vivo experimentation with LCMV in non-human primates showed a significant difference between intravenous and intragastric routes of inoculation $[155,182]$. Additionally, access to cellular receptors differs with exposure to different surfaces of the epithelia $[138,139]$. Furthermore, in vitro studies have shown that entry and release from polarized epithelia is viral specific, and location of viral receptor was indication for direction of viral entry [138, 139]. Here, we investigated the patterns of entry and release when these viruses were exposed to the apical or the basolateral surface of polarized Caco-2 cells. Interestingly, we discovered that LCMV-Armstrong and WE, despite having different pathogenic potential in Non-human primates (NHPs), entered and released via a similar pattern in Caco-2 cells. When LCMV-ARM and LCMV-WE were exposed to the apical side of the intestinal epithelia, mimicking an intragastric infection, we observed high replication and release from the apical surface of these cells, and almost equal release from the basolateral surface. Additionally, when exposed to the basolateral surface of polarized Caco-2 cells, LCMV and MOPV entered apically and released primarily from the apical side, with very little release observed from the basolateral side. Furthermore, we found that ML-29, a LASV surrogate mimicking LASV interaction with susceptible cells, had a different pattern of replication from its parent MOPV strain. When exposed to the apical side of these polarized cells, ML-29 released primarily from 
the apical surface with little to no egress on the basolateral side. Interestingly, when exposed basolaterally, ML-29 showed a delay in viral replication, and did not release infectious progeny until day 5 post infection. The release was observed primarily on the apical surface. Successful entry and release of viral progeny was seen with LCMV and MOPV on both the apical and basolateral side, and only on the apical surface of these cells, indicating that alternative cellular receptors may be associated with entry into polarized intestinal epithelia. Therefore, further investigation into the usage of alternative receptors Axl, DCSIGN, and TYRO-3, should be completed. Our immunofluorescence staining showed location of $\alpha-D G$ to be primarily located on the basolateral side; but fixed cells represent only a snapshot of the cellular environment, thus the characterization of cellular receptors in real-time may be a useful addition toward fully understanding the interaction of these viral-receptor interactions

To further characterize attachment efficiency of the viruses to the apical and basolateral sides of Caco-2 cells, attachment efficacy was measured by qRT/PCR. Interestingly, a lower attachment of ML-29 to the basolateral surface of these cells (the surface of the polarized epithelia in which ML-29 failed to produce progeny), was observed, as opposed to the apical side of cells.

Comparatively, LCMV and MOPV attached similarly, with almost equal ratios to the apical and basolateral surfaces of these cells. The MOPV backbone used in ML-29, is genetically very similar to that of LASV, compared to other psudotype particles with VSV- or retro-based particles. Additionally, ML-29 has GP1, the attachment protein, that is identical to LASV, making ML-29 the best model to 
mimic LASV-cell interaction. However, differences in intracellular trafficking between ML-29 and LASV has yet to be shown. This is most likely due to the K272E substitution, and the mutation located between two peptides at the $\mathrm{N}$ terminal of the GP2 $[77,85,184,185,205]$. This mutation may impact the fusion, and/or post fusion events of ML-29, resulting in different patterns of replication in polarized Caco-2 cells infected with LASV. Interestingly, GP2 mutation in the JUNV vaccine strain Candid \#1 appears to be involved in the destabilization of the metastable GP conformation, contributing to attenuation [233]. The Candid \#1 GP2 was significantly less affected by $\mathrm{NH} 4 \mathrm{Cl}$ (to block the endosomal acidification) than wild type GP2 [233]. The key role of Candid \#1 GP2 mutant was recently confirmed by reverse genetics [234] and this mutation was proposed to be a molecular signature of JUNV attenuation [233]. Additionally, the dynamic interactions of GP1 with cellular receptors, should be investigated. Previous research has determined the crystal structure of LCMV-ARM and LCMV-WE, finding that there is no significant differences between the structure of these two GP1 proteins [209]. However, other studies suggest that LCMVARM does not maintain as strong an affinity to $\alpha-D G$, as LCMV-WE [25].

In the in vitro Caco-2 model of intestinal epithelia, the strains of LCMV, Armstrong and WE, were compared to mimic intravenous versus intragastric inoculation of these virsuses. These two strains of virus, though genetically very similar, have different pathogenic potentials in animal models, including nonhuman primates. While LCMV-ARM-infected non-human primates develop subclinical infection, LCMV-WE-infected monkeys develop severe LF-like fatal 
hepatitis. The LCMV-WE infection results in elevated AST and ALT levels, severe liver pathology, and high viremia $[153,155]$. However, when intragastrically infected with these two strains of LCMV, animals responded in a dose dependent manner. With a low dose of LCMV-WE, most animals did not show signs of disease manifestation compared to intravenous inoculation [155]. However, animals that were infected intragastrically with a very high dose of WE, resulted in manifested disease or died $[155,182]$. Nevertheless, when challenged with lethal levels of WE intravenously, animals were protected against fatal challenge [182]. To further characterize the differences between these two strains of LCMV, we utilized Caco-2 cells to identify how these viruses differed in polarized intestinal epithelia. Interestingly, these strains showed similar phenotypes in their replication in our polarized Caco-2 model. Additionally, we found that viral attachment to the apical and basolateral surfaces of the Caco-2 cells was very similar between the two viruses, although LCMV-ARM did seem to prefer the apical surface. However, the question still lies as to why these two strains cause such different phenotypes in NHPs. Since the attachment efficiency to polarized cells, and the phenotype of replication was not different between the two viruses, then investigation into the intracellular trafficking needed to be completed.

In order investigate these trafficking patterns, we utilized several chemical inhibitors, as well as confocal microscopy staining, to identify potential differences between LCMV-ARM and WE during intracellular trafficking in epithelial and macrophage cell lines. Macrophages are among the first cells to 
come in contact with the virus after crossing of the epithelial barrier. However, it has been shown that LASV prevents activation of macrophages and dendritic cells (DCs) in culture [106, 107], signified by lack of immune mediators and costimulatory molecules such as TNF- $\alpha$, IL-1 $\beta$, IL-6, IL-19, CD 40, CD 54, CD 80, and CD86 [106]. Additionally, LCMV-WE and LASV have been shown to downregulate innate pro-inflammatory responses in vitro and in vivo, and are able to inhibit Toll-like receptor 2 (TLR2)/MyD88 adaptor-like (Mal)-dependent cytokines, as compared to LCMV-ARM and MOPV $[90,102,153,156,167]$. Here, when RAW264.7 cells were infected, LCMV-ARM induced IL-6, compared to no response in LCMV-WE-infected cells. Additionally, when TLR-2 was silenced in RAW264.7 cells, LCMV-ARM-infected macrophages were unable to produce IL6, much like that of WE-infected macrophages. Furthermore, LCMV-ARM colocalization with TLR-2 and EEA1, as opposed to LCMV-WE, indicating that LCMV-WE does not utilize early endosomes during infection of macrophages. Furthermore, we investigated the presence of LCMV-ARM and WE colocalizing with late endosome/lysosomal markers as well. Interestingly, we found colocalization patterns in both LCMV-ARM- and LCMV-WE-infected cells, although a stronger colocalization pattern was seen for ARM infected cells, signifying that both LCMV-ARM and WE utilize the late endosome during entry.

Furthermore, we identified that LCMV-WE was more dependent on membrane cholesterol utilization in VeroE6 and polarized/non-polarized Caco-2 cells, as compared to LCMV-ARM. This signifies that there may be potential differences between how these two viruses utilize and interact with their 
receptors and cell membranes during viral attachment and entry. However, when microtubules were disrupted through the use of Nocodazole, LCMV-ARM and WE were both impacted by the drug, however, the difference of inhibition between the two viruses was not significant, indicating that these two viruses utilize microtubules in a similar manner.

The most interesting finding during these experiments was the effect of bafilomycin treatment. Bafilomycin, an inhibitor of late endosome acidification, was present prior to, during, and after infection, in a number of concentrations. Interestingly, we saw significant differences between how LCMV-ARM and LCMV-WE were impacted by late endosome acidification. LCMV-Armstrong was more highly sensitive to blocking the acidification of the late endosome, than LCMV-WE. A similar observation was observed between LASV and MOPV [225]. Non-pathogenic MOPV was very sensitive to ammonium chloride treatments as compared to LASV, signifying the ability of LASV to release viral RNA faster than that of MOPV [225]. These results indicate that LCMV-WE is capable of allowing the virus to fuse and continue with replication faster than that of LCMV-ARM. This ability to fuse during more basic environments signifies that LCMV-WE may not need to proceed to the late endosome or lysosome before releasing viral RNA into the cytoplasm, resulting in a potential to release infectious progeny sooner, and manipulate or bypass innate immune regulation, as seen with our TLR-2 interactions in macrophages. 


\section{FUTURE DIRECTIONS}

ML-29 is a desirable candidate as a LASV vaccine. Several studies have been used to identify the protective capacity of this reassortant virus on LASV challenges $[84,85,87,91]$. ML-29 is capable of protecting against several strains of LASV, and shows potential to have some cross-protective tendencies for LCMV-WE [87]. A large percentage of the population in LASV-endemic regions are seropositive for the virus and do not show clinical signs and symptoms of the disease. Further studies into how WT LASV enters, releases, and attaches to polarized Caco-2 cells would provide valuable knowledge for the field to better understand potential factors resulting in seropositivity and protection against LASV infection and disease in endemic regions. Additionally, due to the results that we have observed with ML-29 in vitro, along with the in vivo challenge studies, it would be interesting to investigate how the route of vaccination influences the protective abilities of ML-29. Intravenous and subcutaneous methods of vaccination have been investigated, however, intragastric inoculation has yet to be explored. Additionally, intragastric routes of inoculation in a mouse, guinea pig, and/or a NHP model has yet to be investigated with LASV-infected animals. Similar studies have been done with the prototypic arenavirus, LCMV, utilizing different strains to determine differences in pathogenicity $[155,182]$, however, LASV has yet to be investigated.

Caco-2 cells, as an intestinal epithelial model, are useful in determining the cellular interactions of these mammalian arenaviruses. However, these cells 
to not mimic a complete model of the intestinal environment. Although Caco-2 cells polarize and develop into enterocyte-like cells, this model only depicts one aspect of the in vivo environment. The intestinal environment is a highly immune-active site of the body and through our cell culture model, we are unable to determine the role of immune regulators such as Dendritic cells (DCs), macrophages, $\mathrm{M}$ cells, and Peyers patches. The Caco-2 cell culture model mimics only the intestinal epithelia, not allowing us to investigate other key players that may have an impact on how these viruses are interacting with the intestine during intragastric infection. We did discover, that LCMV and MOPV primarily release to the intestinal lumen; however, there was a slight release of infectious progeny to the basolateral side. However, when tested in vivo, intragastric inoculation resulted in no detectible virus in any tissues collected from the experiments [155], bringing to question, the role of immune cells present at the site of inoculation. A potential experimental setup to study these interactions may be to utilize a co-culture model. While Caco-2 cells are grown on transwell inserts, macrophages would be cultured in the bottom transwells, and the intestinal epithelia would be infected with different strains of LCMV. Moreover, investigating the infection of macrophages and determining how these infections may impact epithelial barrier integrity. Furthermore, LASV has been known to downregulate and inhibit INF responses and prevent macrophages and DCs from activating. It would be of interest to determine how LASV interacts with these immune regulators of the digestive system in order to further and more 
clearly understand the seropositive-phenotype that is observed in LASV-endemic populations.

While LASV does indeed infect a large number of the population, only a small subset of this population results in clinical manifestations of disease, and an even smaller population dies annually from these infections. However, what has yet to be identified is what differentiates populations that are seropositive with subclinical infections, from those that succumb to infection that results in severe illness and even death. Western Africa is not just home to LASV; several other viruses including Ebola, Marburg virus, Rift Valley Fever, Crimean-Congo hemorrhagic fever, flaviviruses, and alphaviruses reside in this region [235]. In addition, influenza, cholera, and malaria have been identified as human pathogens in West Africa. Recent studies investigating concurrent infection of malaria and dengue viral infection have been investigated, and co-infection of these two pathogens is higher than initially thought, and clinical symptoms are likely be more severe than single infection [236]. Additionally, a preliminary seroepidemiology study of LASV and HIV shows that there is potential for connection between these two viruses. However, there has yet to be controlled experimentation as to how these other diseases could potentially play a role in LASV pathogenicity. One potential factor that should be considered is the role of the intestinal and bronchial barriers during LASV infection. For example, if a patient has been infected with an agent that causes intestinal barrier disruption, this may play a potential role in the viral entry and its ability to develop systemic infection. In the case of LCMV-ARM and WE in rhesus macaques, LCMV-WE 
induced systemic infection with fatal outcome, however when intragastrically infected, this virus was not capable of systemic disease, but rather a localized infection. It would be of interest to investigate how this barrier system plays a role in keeping a localized infection in NHPs or other animal models during LCMV and LASV infection. Additionally, these experiments may provide insight as to why such a high seroprevalence of LASV is present in Western Africa and why not all patients succumb to disease.

While in our hands, ML-29 did not have successful replication in MadinDarby Canine Kidney (MDCK) epithelial cells, however, previous studies have shown LASV's ability to replicate sufficiently in these cells [138]. Additionally, bronchial epithelia have been used to study LCMV [139], however, in our Human bronchial epithelial (HBE) model, we had insufficient replication of LCMV-ARM and did not further characterize other viruses in this model. It would be of interest however, to utilize a number of different polarized epithelial types to determine how LCMV-ARM, LCMV-WE, MOPV, and ML-29 replicate in these other cell types. This would provide further support for our hypothesis that arenavirus replication is host, virus, and tissue specific. By investigating how these viruses interact with different tissues, this may further explain the seropositivity seen with small populations in West African villages. Additionally, these phenotypes would provide further support for ML-29 as a vaccine candidate. For example, while we did not see sufficient release to the basolateral surface of the Caco-2 cells with ML-29, we saw release only from the apical surface of these cells. In return, this may be a beneficial aspect of ML-29 
as a vaccine candidate, providing only localized intestinal infection, resulting in the immune system having exposure to and time to mount sufficient and producing neutralizing immune responses.

Additionally, New World (NW) mammalian arenaviruses, though related to the OW group, have differences in pathogenic phenotypes, receptor usage, and global location. However, these NW viruses are transmitted through their rodent reservoirs in the same way as their OW relatives. It would be of interest to utilize the Caco-2 model developed her for OW arenaviral infection, to investigate the patterns of replication with NW viruses. Due to the differences in receptor usage, there may be interesting findings in how these viruses replicate and utilize intestinal epithelia in vitro. Furthermore, while there is a successful vaccine for Junin virus, Candid \# 1, there has yet to be a vaccine created for Machupo, another highly pathogenic NW arenavirus. By investigating these interactions with the intestinal epithelia, there may be interesting findings that may benefit the development of a new Machupo vaccine.

We have identified several differences in the intracellular trafficking between LCMV-ARM and WE. However, we have not exhausted the viral replication of these two viruses. It would be of interest to determine intracellular trafficking with additional inhibitors. Manipulation of the multivesicular bodies of host cells during arenaviral infection has been identified as part of the arenaviral life cycle. Multivesicular bodies are able to be disrupted using the PI3KI inhibitor wortmannin. Additionally, it would be of interest to knockdown late endosome 
fusion of LAMP1 and determine its effect of viral replication between ARM and WE to provide additional support for our confocal microscopy images. 


\section{REFERENCES}

1. Rowe, W.P., et al., Arenoviruses: proposed name for a newly defined virus group. J Virol, 1970. 5(5): p. 651-2.

2. Bodewes, R., et al., Detection of novel divergent arenaviruses in boid snakes with inclusion body disease in The Netherlands. J Gen Virol, 2013. 94(Pt 6): p. 1206-10.

3. Radoshitzky, S.R., et al., Past, present, and future of arenavirus taxonomy. Archives of Virology, 2015. 160(7): p. 1851-1874.

4. Bowen, M.D., C.J. Peters, and S.T. Nichol, The phylogeny of New World (Tacaribe complex) arenaviruses. Virology, 1996. 219(1): p. 285-90.

5. McLay, L., Y. Liang, and H. Ly, Comparative analysis of disease pathogenesis and molecular mechanisms of New World and Old World arenavirus infections. J Gen Virol, 2014. 95(Pt 1): p. 1-15.

6. Charrel, R.N., X. de Lamballerie, and S. Emonet, Phylogeny of the genus Arenavirus. Curr Opin Microbiol, 2008. 11(4): p. 362-8.

7. Lecompte, E., et al., Genetic identification of Kodoko virus, a novel arenavirus of the African pigmy mouse (Mus Nannomys minutoides) in West Africa. Virology, 2007. 364(1): p. 178-83.

8. Palacios, G., et al., $A$ new arenavirus in a cluster of fatal transplantassociated diseases. N Engl J Med, 2008. 358(10): p. 991-8.

9. Grove, J. and M. Marsh, The cell biology of receptor-mediated virus entry. J Cell Biol, 2011. 195(7): p. 1071-82.

10. Cao, W., et al., Identification of alpha-dystroglycan as a receptor for lymphocytic choriomeningitis virus and Lassa fever virus. Science, 1998. 282(5396): p. 2079-81.

11. Spiropoulou, C.F., et al., New World Arenavirus Clade $C$, but Not Clade $A$ and $B$ Viruses, Utilizes -Dystroglycan as Its Major Receptor. Journal of Virology, 2002. 76(10): p. 5140-5146.

12. Yoshida-Moriguchi, T. and K.P. Campbell, Matriglycan: a novel polysaccharide that links dystroglycan to the basement membrane. Glycobiology, 2015. 25(7): p. 702-13.

13. Kunz, S., et al., Posttranslational modification of alpha-dystroglycan, the cellular receptor for arenaviruses, by the glycosyltransferase LARGE is critical for virus binding. J Virol, 2005. 79(22): p. 14282-96.

14. Rojek, J.M., et al., Old World arenavirus infection interferes with the expression of functional alpha-dystroglycan in the host cell. Mol Biol Cell, 2007. 18(11): p. 4493-507.

15. Hara, Y., et al., Like-acetylglucosaminyltransferase (LARGE)-dependent modification of dystroglycan at Thr-317/319 is required for laminin binding and arenavirus infection. Proc Natl Acad Sci U S A, 2011. 108(42): p. 17426-31.

16. Sabeti, P.C., et al., Genome-wide detection and characterization of positive selection in human populations. Nature, 2007. 449(7164): p. 9138. 
17. Andersen, K.G., et al., Clinical Sequencing Uncovers Origins and Evolution of Lassa Virus. Cell, 2015. 162(4): p. 738-50.

18. Andersen, K.G., et al., Genome-wide scans provide evidence for positive selection of genes implicated in Lassa fever. Philos Trans R Soc Lond B Biol Sci, 2012. 367(1590): p. 868-77.

19. Rojek, J.M., et al., Binding of Lassa virus perturbs extracellular matrixinduced signal transduction via dystroglycan. Cell Microbiol, 2012. 14(7): p. 1122-34.

20. Kunz, S., et al., Use of alternative receptors different than $\alpha$-dystroglycan by selected isolates of lymphocytic choriomeningitis virus. Virology, 2004. 325(2): p. 432-445.

21. Oldstone, M.B. and K.P. Campbell, Decoding arenavirus pathogenesis: essential roles for alpha-dystroglycan-virus interactions and the immune response. Virology, 2011. 411(2): p. 170-9.

22. Oldstone, M., Biology and pathogenesis of lymphocytic choriomeningitis virus infection, in Arenaviruses II. 2002, Springer. p. 83-117.

23. Sullivan, B.M., et al., Point mutation in the glycoprotein of lymphocytic choriomeningitis virus is necessary for receptor binding, dendritic cell infection, and long-term persistence. Proceedings of the National Academy of Sciences, 2011. 108(7): p. 2969-2974.

24. Sevilla, N., et al., Immunosuppression and resultant viral persistence by specific viral targeting of dendritic cells. Journal of Experimental Medicine, 2000. 192(9): p. 1249-1260.

25. Smelt, S.C., et al., Differences in affinity of binding of lymphocytic choriomeningitis virus strains to the cellular receptor alpha-dystroglycan correlate with viral tropism and disease kinetics. J Virol, 2001. 75(1): p. 448-57.

26. Teng, M.N., et al., A single amino acid change in the glycoprotein of lymphocytic choriomeningitis virus is associated with the ability to cause growth hormone deficiency syndrome. Journal of virology, 1996. 70(12): p. 8438-8443.

27. Hastie, K.M., et al., Crystal Structure of the Oligomeric Form of Lassa Virus Matrix Protein Z. J Virol, 2016. 90(9): p. 4556-62.

28. Jae, L.T., et al., Virus entry. Lassa virus entry requires a trigger-induced receptor switch. Science, 2014. 344(6191): p. 1506-10.

29. Cohen-Dvashi, H., et al., Molecular mechanism for LAMP1 recognition by Lassa virus. Journal of virology, 2015. 89(15): p. 7584-7592.

30. Shimojima, M., et al., Identification of cell surface molecules involved in dystroglycan-independent Lassa virus cell entry. J Virol, 2012. 86(4): p. 2067-78.

31. Shimojima, M. and Y. Kawaoka, Cell Surface Molecules Involved in Infection Mediated by Lymphocytic Choriomeningitis Virus Glycoprotein. Journal of Veterinary Medical Science, 2012. 74(10): p. 1363-1366.

32. Lemke, G. and C.V. Rothlin, Immunobiology of the TAM receptors. Nat Rev Immunol, 2008. 8(5): p. 327-36.

33. Lemke, G. and T. Burstyn-Cohen, TAM receptors and the clearance of apoptotic cells. Ann N Y Acad Sci, 2010. 1209: p. 23-9.

34. Rojek, J.M. and S. Kunz, Cell entry by human pathogenic arenaviruses. Cell Microbiol, 2008. 10(4): p. 828-35. 
Rojek, J.M., et al., Different mechanisms of cell entry by humanpathogenic Old World and New World arenaviruses. J Virol, 2008. 82(15): p. 7677-87.

36. Rojek, J.M., M. Perez, and S. Kunz, Cellular entry of lymphocytic choriomeningitis virus. J Virol, 2008. 82(3): p. 1505-17.

37. Shah, W.A., H. Peng, and S. Carbonetto, Role of non-raft cholesterol in lymphocytic choriomeningitis virus infection via alpha-dystroglycan. J Gen Virol, 2006. 87(Pt 3): p. 673-8.

38. Vela, E.M., et al., Arenavirus entry occurs through a cholesteroldependent, non-caveolar, clathrin-mediated endocytic mechanism. Virology, 2007. 369(1): p. 1-11.

39. Moraz, M.L., et al., Cell entry of Lassa virus induces tyrosine phosphorylation of dystroglycan. Cell Microbiol, 2013. 15(5): p. 689-700.

40.

41.

42. Iwasaki, M., N. Ngo, and J.C. de la Torre, Sodium hydrogen exchangers contribute to arenavirus cell entry. J Virol, 2014. 88(1): p. 643-54. Mercer, J. and A. Helenius, Gulping rather than sipping: macropinocytosis as a way of virus entry. Curr Opin Microbiol, 2012. 15(4): p. 490-9.

Scott, C.C., F. Vacca, and J. Gruenberg, Endosome maturation, transport and functions. Semin Cell Dev Biol, 2014. 31: p. 2-10.

43. Pasqual, G., et al., Old world arenaviruses enter the host cell via the multivesicular body and depend on the endosomal sorting complex required for transport. PLoS Pathog, 2011. 7(9): p. e1002232.

44. Quirin, K., et al., Lymphocytic choriomeningitis virus uses a novel endocytic pathway for infectious entry via late endosomes. Virology, 2008. 378(1): p. 21-33.

45. McCormick, J.B. and S.P. Fisher-Hoch, Lassa fever. Curr Top Microbiol Immunol, 2002. 262: p. 75-109.

46. Eskelinen, E.L., Roles of LAMP-1 and LAMP-2 in lysosome biogenesis and autophagy. Mol Aspects Med, 2006. 27(5-6): p. 495-502.

47. Andrejewski, N., et al., Normal lysosomal morphology and function in LAMP-1-deficient mice. J Biol Chem, 1999. 274(18): p. 12692-701.

48. Cohen-Dvashi, H., et al., Role of LAMP1 Binding and pH Sensing by the Spike Complex of Lassa Virus. J Virol, 2016. 90(22): p. 10329-10338.

49. Israeli, $\mathrm{H}$., et al., Mapping of the Lassa virus LAMP1 binding site reveals unique determinants not shared by other old world arenaviruses. PLoS pathogens, 2017. 13(4): p. e1006337.

50. Hulseberg, C.E., et al., Lamp1 Increases the Efficiency of Lassa Virus Infection by Promoting Fusion in Less Acidic Endosomal Compartments. mBio, 2018. 9(1): p. e01818-17.

51. Hoenen, T., L. Kolesnikova, and S. Becker, Recent advances in filovirusand arenavirus-like particles. Future Virology, 2007. 2(2): p. 193-203.

52. Wolff, S., H. Ebihara, and A. Groseth, Arenavirus budding: a common pathway with mechanistic differences. Viruses, 2013. 5(2): p. 528-49.

53. Martinez-Sobrido, L., et al., Inhibition of the type I interferon response by the nucleoprotein of the prototypic arenavirus lymphocytic choriomeningitis virus. J Virol, 2006. 80(18): p. 9192-9.

54. Martinez-Sobrido, L., et al., Differential inhibition of type I interferon induction by arenavirus nucleoproteins. J Virol, 2007. 81(22): p. 12696703.

55. Goni, S.E., et al., Genomic features of attenuated Junin virus vaccine strain candidate. Virus Genes, 2006. 32(1): p. 37-41. 
56.

Johnson, K.M., S.B. Halstead, and S.N. Cohen, Hemorrhagic fevers of Southeast Asia and South America: a comparative appraisal. Prog Med Virol, 1967. 9: p. 105-58.

57. Murphy, B.R. and R. Webster, Fields virology. Fields virology, 1996.

58. Buchmeier MJ, P.C., de la Torre JC., Arenaviridae: the virus and their replication. Fields Virology, 2007. 2: p. 1792-1827.

59. Meyer BJ, d.L.T.J., Southern PJ. , Arenaviruses: Genomic RNAs, Transcription, and Replication. Oldstone MB, ed. Arenaviruses I, 2002. 262: p. 139-149.

60. Pfaffl, M.W., et al., Development of clenbuterol reference materials: lyophilized bovine eye samples free of clenbuterol (CRM 673) and containing clenbuterol (CRM 674). Part 2: certification. Fresenius J Anal Chem, 2001. 371(8): p. 1092-7.

61. Buchmeier, M.J., et al., The virology and immunobiology of lymphocytic choriomeningitis virus infection. Adv Immunol, 1980. 30: p. 275-331.

62. Stenglein, M.D., et al., Identification, characterization, and in vitro culture of highly divergent arenaviruses from boa constrictors and annulated tree boas: candidate etiological agents for snake inclusion body disease. MBio, 2012. 3(4): p. e00180-12.

63. Young, P.R. and C.R. Howard, Fine structure analysis of Pichinde virus nucleocapsids. J Gen Virol, 1983. 64 (Pt 4): p. 833-42.

64. PJ, S., Arenaviridae: The viruses and their replicaiton. Fields virology, 1996: p. 1505-1519.

65. Eichler, R., et al., Identification of Lassa virus glycoprotein signal peptide as a trans-acting maturation factor. EMBO reports, 2003. 4(11): p. 10841088.

66. Eichler, R., et al., Signal peptide of Lassa virus glycoprotein GP-C exhibits an unusual length. FEBS letters, 2003. 538(1-3): p. 203-206.

67. York, J., et al., The signal peptide of the Junin arenavirus envelope glycoprotein is myristoylated and forms an essential subunit of the mature G1-G2 complex. Journal of virology, 2004. 78(19): p. 10783-10792.

68. York, J. and J.H. Nunberg, Role of the stable signal peptide of Junin arenavirus envelope glycoprotein in $\mathrm{pH}$-dependent membrane fusion. Journal of virology, 2006. 80(15): p. 7775-7780.

69. York, J. and J.H. Nunberg, Distinct requirements for signal peptidase processing and function in the stable signal peptide subunit of the Junin virus envelope glycoprotein. Virology, 2007. 359(1): p. 72-81.

70. Froeschke, M., et al., Long-lived signal peptide of lymphocytic choriomeningitis virus glycoprotein pGP-C. Journal of Biological Chemistry, 2003. 278(43): p. 41914-41920.

71. Kunz, S., et al., Mechanisms for lymphocytic choriomeningitis virus glycoprotein cleavage, transport, and incorporation into virions. Virology, 2003. 314(1): p. 168-178.

72. Saunders, A.A., et al., Mapping the landscape of the lymphocytic choriomeningitis virus stable signal peptide reveals novel functional domains. Journal of virology, 2007. 81(11): p. 5649-5657.

73. Schrempf, S., et al., Signal peptide requirements for lymphocytic choriomeningitis virus glycoprotein $C$ maturation and virus infectivity. Journal of virology, 2007. 81(22): p. 12515-12524. 
74. Eschli, B., et al., Identification of an N-terminal trimeric coiled-coil core within arenavirus glycoprotein 2 permits assignment to class I viral fusion proteins. J Virol, 2006. 80(12): p. 5897-907.

75. Parsy, M.L., et al., Crystal structure of Venezuelan hemorrhagic fever virus fusion glycoprotein reveals a class 1 postfusion architecture with extensive glycosylation. J Virol, 2013. 87(23): p. 13070-5.

76. Igonet, S., et al., X-ray structure of the arenavirus glycoprotein GP2 in its postfusion hairpin conformation. Proc Natl Acad Sci U S A, 2011. 108(50): p. 19967-72.

77. Klewitz, C., H.D. Klenk, and J. ter Meulen, Amino acids from both Nterminal hydrophobic regions of the Lassa virus envelope glycoprotein GP-2 are critical for $\mathrm{pH}$-dependent membrane fusion and infectivity. J Gen Virol, 2007. 88(Pt 8): p. 2320-8.

78. Khan, S.H., et al., New opportunities for field research on the pathogenesis and treatment of Lassa fever. Antiviral Res, 2008. 78(1): p. 103-15.

79. Fichet-Calvet, E. and D.J. Rogers, Risk maps of Lassa fever in West Africa. PLoS Negl Trop Dis, 2009. 3(3): p. e388.

80. Loureiro, M.E., et al., Molecular determinants of arenavirus $Z$ protein homo-oligomerization and L polymerase binding. J Virol, 2011. 85(23): p. 12304-14.

81. Richmond, J.K. and D.J. Baglole, Lassa fever: epidemiology, clinical features, and social consequences. Bmj, 2003. 327(7426): p. 1271-5.

82. Buchmeier, M., Bowen, M. \& Peters, C., Arenaviridae: the viruses and their replication. Fields Virology 4th Edition, 2001.

83. McCormick, J.B., et al., Lassa fever. Effective therapy with ribavirin. N Engl J Med, 1986. 314(1): p. 20-6.

84. Goicochea, M.A., et al., Evaluation of Lassa virus vaccine immunogenicity in a CBA/J-ML29 mouse model. Vaccine, 2012. 30(8): p. 1445-52.

85. Lukashevich, I.S., et al., A live attenuated vaccine for Lassa fever made by reassortment of Lassa and Mopeia viruses. J Virol, 2005. 79(22): p. 13934-42.

86. Barkar, N.D. and I.S. Lukashevich, [Lassa and Mozambique viruses: cross protection in experiments on mice and action of immunosuppressants on experimental infections]. Vopr Virusol, 1989. 34(5): p. 598-603.

87. Carrion, R., Jr., et al., A ML29 reassortant virus protects guinea pigs against a distantly related Nigerian strain of Lassa virus and can provide sterilizing immunity. Vaccine, 2007. 25(20): p. 4093-102.

88. Lukashevich, I.S., et al., Safety, immunogenicity, and efficacy of the ML29 reassortant vaccine for Lassa fever in small non-human primates.

Vaccine, 2008. 26(41): p. 5246-54.

89. Zapata, J.C., et al., An attenuated Lassa vaccine in SIV-infected rhesus macaques does not persist or cause arenavirus disease but does elicit Lassa virus-specific immunity. Virol J, 2013. 10: p. 52.

90. Carrion, R., Jr., et al., Lassa virus infection in experimentally infected marmosets: liver pathology and immunophenotypic alterations in target tissues. J Virol, 2007. 81(12): p. 6482-90.

91. Moshkoff, D.A., M.S. Salvato, and I.S. Lukashevich, Molecular characterization of a reassortant virus derived from Lassa and Mopeia viruses. Virus Genes, 2007. 34(2): p. 169-76. 
92. Monath, T.P. and J. Casals, Diagnosis of Lassa fever and the isolation and management of patients. Bull World Health Organ, 1975. 52(4-6): $p$. 707-15.

93. McCormick, J.B., et al., A case-control study of the clinical diagnosis and course of Lassa fever. J Infect Dis, 1987. 155(3): p. 445-55.

94. Moraz, M.L. and S. Kunz, Pathogenesis of arenavirus hemorrhagic fevers. Expert Rev Anti Infect Ther, 2011. 9(1): p. 49-59.

95. Cummins, D., et al., Acute sensorineural deafness in Lassa fever. JAMA, 1990. 264(16): p. 2093-6.

96. McCormick, J.B., et al., Lassa virus hepatitis: a study of fatal Lassa fever in humans. Am J Trop Med Hyg, 1986. 35(2): p. 401-7.

97. McCormick, J.B., Clinical, epidemiologic, and therapeutic aspects of Lassa fever. Med Microbiol Immunol, 1986. 175(2-3): p. 153-5.

98. Johnson, K.M., et al., Clinical virology of Lassa fever in hospitalized patients. J Infect Dis, 1987. 155(3): p. 456-64.

99. Walker, D.H., et al., Pathologic and virologic study of fatal Lassa fever in man. Am J Pathol, 1982. 107(3): p. 349-56.

100. Roberts, P.J., et al., Plasma from patients with severe Lassa fever profoundly modulates $f$-met-leu-phe induced superoxide generation in neutrophils. Br J Haematol, 1989. 73(2): p. 152-7.

101. Fisher-Hoch, S., et al., Hematologic dysfunction in Lassa fever. J Med Virol, 1988. 26(2): p. 127-35.

102. Lukashevich, I.S., et al., Lassa and Mopeia virus replication in human monocytes/macrophages and in endothelial cells: different effects on IL-8 and TNF-alpha gene expression. J Med Virol, 1999. 59(4): p. 552-60.

103. Walker, D.H., et al., Experimental infection of rhesus monkeys with Lassa virus and a closely related arenavirus, Mozambique virus. J Infect Dis, 1982. 146(3): p. 360-8.

104. Paessler, S. and D.H. Walker, Pathogenesis of the viral hemorrhagic fevers. Annu Rev Pathol, 2013. 8: p. 411-40.

105. Mahanty, S., et al., Low levels of interleukin-8 and interferon-inducible protein-10 in serum are associated with fatal infections in acute Lassa fever. J Infect Dis, 2001. 183(12): p. 1713-21.

106. Baize, S., et al., Lassa virus infection of human dendritic cells and macrophages is productive but fails to activate cells. J Immunol, 2004. 172(5): p. 2861-9.

107. Mahanty, S., et al., Cutting edge: impairment of dendritic cells and adaptive immunity by Ebola and Lassa viruses. J Immunol, 2003. 170(6): p. 2797-801.

108. Rivers, T.M. and T.F. McNair Scott, Meningitis in Man Caused by a Filterable Virus. Science, 1935. 81(2105): p. 439-40.

109. Traub, E., A Filterable Virus Recovered from White Mice. Science, 1935. 81(2099): p. 298-9.

110. Scott, T.F. and T.M. Rivers, Meningitis in Man Caused by a Filterable Virus : I. Two Cases and the Method of Obtaining a Virus from Their Spinal Fluids. J Exp Med, 1936. 63(3): p. 397-414.

111. Childs, J.E. and C.J. Peters, Ecology and epidemiology of arenaviruses and their hosts, in The Arenaviridae. 1993, Springer. p. 331-384.

112. $\quad$ CDC, MMWR Morb. Mortal Wkly Rep. 54, 2005: p. 537-539.

113. Fischer, S.A., et al., Transmission of lymphocytic choriomeningitis virus by organ transplantation. N Engl J Med, 2006. 354(21): p. 2235-49. 
114. Macneil, A., et al., Solid organ transplant-associated lymphocytic choriomeningitis, United States, 2011. Emerg Infect Dis, 2012. 18(8): p. 1256-62.

115. MB, G., Bulliten. WHO 52. 1975: p. 549-553.

116. Hotchin, J., et al., Lymphocytic choriomeningitis in a hamster colony causes infection of hospital personnel. Science, 1974. 185(4157): p. 1173-1174.

117. Childs, J.E., et al., Human-rodent contact and infection with lymphocytic choriomeningitis and Seoul viruses in an inner-city population. The American journal of tropical medicine and hygiene, 1991. 44(2): p. 117121.

118. Childs, J.E., et al., Lymphocytic choriomeningitis virus infection and house mouse (Mus musculus) distribution in urban Baltimore. The American journal of tropical medicine and hygiene, 1992. 47(1): p. 27-34.

119. Park, J., et al., Development of an RT-PCR assay for diagnosis of lymphocytic choriomeningitis virus (LCMV) infection and its use in a prospective surveillance study. J Med Virol, 1997. 1997: p. 107-114.

120. Stephensen, C.B., et al., Prevalence of serum antibodies against lymphocytic choriomeningitis virus in selected populations from two U.S. cities. J Med Virol, 1992. 38(1): p. 27-31.

121. Dobec, M., et al., High prevalence of antibodies to lymphocytic choriomeningitis virus in a murine typhus endemic region in Croatia. Journal of medical virology, 2006. 78(12): p. 1643-1647.

122. Reiserová, L., et al., Identification of MaTu-MX agent as a new strain of lymphocytic choriomeningitis virus (LCMV) and serological indication of horizontal spread of LCMV in human population. Virology, 1999. 257(1): p. 73-83.

123. Marrie, T.J. and M.-F. Saron, Seroprevalence of lymphocytic choriomeningitis virus in Nova Scotia. The American journal of tropical medicine and hygiene, 1998. 58(1): p. 47-49.

124. Ackermann, R. and F. Lehmann-Grube, Lymphocytic Choriomeningitis Virus and other Arena Viruses. 1973, Springer-Verlag, Berlin.

125. Riera, L., et al., Serological study of the lymphochoriomeningitis virus (LCMV) in an inner city of Argentina. Journal of medical virology, 2005. 76(2): p. 285-289.

126. Lledó, L., et al., Lymphocytic choriomeningitis virus infection in a province of Spain: analysis of sera from the general population and wild rodents. Journal of medical virology, 2003. 70(2): p. 273-275.

127. Kallio-Kokko, H., et al., Hantavirus and arenavirus antibody prevalence in rodents and humans in Trentino, Northern Italy. Epidemiology \& Infection, 2006. 134(4): p. 830-836.

128. Bonthius, D.J., Lymphocytic choriomeningitis virus: an underrecognized cause of neurologic disease in the fetus, child, and adult. Semin Pediatr Neurol, 2012. 19(3): p. 89-95.

129. Kunz, S. and J.-C. de la Torre, Arenavirus infection in the nervous system: uncovering principles of virus-host interaction and viral pathogenesis. 2008, Cambridge University Press, Cambridge, UK. p. 7593.

130. Jamieson, D.J., et al., Lymphocytic choriomeningitis virus: an emerging obstetric pathogen? American Journal of Obstetrics \& Gynecology, 2006. 194(6): p. 1532-1536. 
131. (CDC), C.f.D.C.a.P., Brief Report: Lymphocytic choriomeningitis virus transmitted through solid organ transplantation-Massachussetts, 2008. MMWR Morb Mortal Wkly Rep, 2008(57): p. 799-801.

132. Barton, L.L. and M.B. Mets, Lymphocytic choriomeningitis virus: pediatric pathogen and fetal teratogen. The Pediatric infectious disease journal, 1999. 18(6): p. 540-541.

133. Barton, L.L., M.B. Mets, and C.L. Beauchamp, Lymphocytic choriomeningitis virus: emerging fetal teratogen. American Journal of Obstetrics \& Gynecology, 2002. 187(6): p. 1715-1716.

134. Bonthius, D.J. and S. Perlman, Congenital viral infections of the brain: lessons learned from lymphocytic choriomeningitis virus in the neonatal rat. PLoS pathogens, 2007. 3(11): p. e149.

135. Wright, R., et al., Congenital lymphocytic choriomeningitis virus syndrome: a disease that mimics congenital toxoplasmosis or Cytomegalovirus infection. Pediatrics, 1997. 100(1): p. E9.

136. Peters, C.J., Lymphocytic choriomeningitis virus--an old enemy up to new tricks. N Engl J Med, 2006. 354(21): p. 2208-11.

137. Ter Meulen, J., et al., Hunting of peridomestic rodents and consumption of their meat as possible risk factors for rodent-to-human transmission of Lassa virus in the Republic of Guinea. Am J Trop Med Hyg, 1996. 55(6): p. 661-6.

138. Schlie, K., et al., Viral protein determinants of Lassa virus entry and release from polarized epithelial cells. J Virol, 2010. 84(7): p. 3178-88.

139. Dylla, D.E., et al., Basolateral entry and release of New and Old World arenaviruses from human airway epithelia. J Virol, 2008. 82(12): p. 60348.

140. Esclatine, A., et al., Human cytomegalovirus infects Caco-2 intestinal epithelial cells basolaterally regardless of the differentiation state. J Virol, 2000. 74(1): p. 513-7.

141. Basak, S. and R.W. Compans, Polarized entry of canine parvovirus in an epithelial cell line. J Virol, 1989. 63(7): p. 3164-7.

142. Tseng, C.T., et al., Apical entry and release of severe acute respiratory syndrome-associated coronavirus in polarized Calu-3 lung epithelial cells. J Virol, 2005. 79(15): p. 9470-9.

143. Krautkramer, E. and M. Zeier, Hantavirus causing hemorrhagic fever with renal syndrome enters from the apical surface and requires decayaccelerating factor (DAF/CD55). J Virol, 2008. 82(9): p. 4257-64.

144. Excoffon, K.J., et al., Reovirus preferentially infects the basolateral surface and is released from the apical surface of polarized human respiratory epithelial cells. J Infect Dis, 2008. 197(8): p. 1189-97.

145. Zurzolo, C., et al., Opposite polarity of virus budding and of viral envelope glycoprotein distribution in epithelial cells derived from different tissues. $\mathrm{J}$ Cell Biol, 1992. 117(3): p. 551-64.

146. Rossen, J.W., et al., A murine and a porcine coronavirus are released from opposite surfaces of the same epithelial cells. Virology, 1996. 224(1): p. 345-51.

147. ter Meulen, J., et al., Characterization of human CD4(+) T-cell clones recognizing conserved and variable epitopes of the Lassa virus nucleoprotein. J Virol, 2000. 74(5): p. 2186-92.

148. Geisbert, T.W., et al., Development of a new vaccine for the prevention of Lassa fever. PLoS Med, 2005. 2(6): p. e183. 
149. Fisher-Hoch, S.P., et al., Effective vaccine for lassa fever. J Virol, 2000. 74(15): p. 6777-83.

150. Meulen, J., et al., Old and New World arenaviruses share a highly conserved epitope in the fusion domain of the glycoprotein 2, which is recognized by Lassa virus-specific human CD4+ T-cell clones. Virology, 2004. 321(1): p. 134-43.

151. Baize, S., et al., Early and strong immune responses are associated with control of viral replication and recovery in lassa virus-infected cynomolgus monkeys. J Virol, 2009. 83(11): p. 5890-903.

152. Branco, L.M., et al., Emerging trends in Lassa fever: redefining the role of immunoglobulin $M$ and inflammation in diagnosing acute infection. Virol $\mathrm{J}$, 2011. 8: p. 478.

153. Lukashevich, I.S., et al., Arenavirus-Mediated Liver Pathology: Acute Lymphocytic Choriomeningitis Virus Infection of Rhesus Macaques Is Characterized by High-Level Interleukin-6 Expression and Hepatocyte Proliferation. Journal of Virology, 2003. 77(3): p. 1727-1737.

154. Hensley, L.E., et al., Pathogenesis of Lassa fever in cynomolgus macaques. Virol J, 2011. 8: p. 205.

155. Lukashevich, I., Hemorrhagic Fever occurs after intravenous but not after intragastric inoculation of rhesus macaques with LCMV. 2002.

156. Lukashevich, I.S., et al., LCMV-mediated hepatitis in rhesus macaques: WE but not ARM strain activates hepatocytes and induces liver regeneration. Arch Virol, 2004. 149(12): p. 2319-36.

157. Pannetier, D., et al., Human macrophages, but not dendritic cells, are activated and produce alpha/beta interferons in response to Mopeia virus infection. J Virol, 2004. 78(19): p. 10516-24.

158. Groseth, A., et al., Tacaribe virus but not junin virus infection induces cytokine release from primary human monocytes and macrophages.

PLoS Negl Trop Dis, 2011. 5(5): p. e1137.

159. Cuevas, C.D. and S.R. Ross, Toll-like receptor 2-mediated innate immune responses against Junin virus in mice lead to antiviral adaptive immune responses during systemic infection and do not affect viral replication in the brain. J Virol, 2014. 88(14): p. 7703-14.

160. Zhou, S., et al., MyD88 is critical for the development of innate and adaptive immunity during acute lymphocytic choriomeningitis virus infection. Eur J Immunol, 2005. 35(3): p. 822-30.

161. Zhou, S., et al., Lymphocytic choriomeningitis virus (LCMV) infection of CNS glial cells results in TLR2-MyD88/Mal-dependent inflammatory responses. J Neuroimmunol, 2008. 194(1-2): p. 70-82.

162. Zhou, S., et al., MyD88 intrinsically regulates CD4 T-cell responses. J Virol, 2009. 83(4): p. 1625-34.

163. Qi, X., et al., Cap binding and immune evasion revealed by Lassa nucleoprotein structure. Nature, 2010. 468(7325): p. 779-83.

164. Hastie, K.M., et al., Crystal structure of the Lassa virus nucleoproteinRNA complex reveals a gating mechanism for RNA binding. Proc Natl Acad Sci U S A, 2011. 108(48): p. 19365-70.

165. Jensen, S. and A.R. Thomsen, Sensing of RNA viruses: a review of innate immune receptors involved in recognizing $R N A$ virus invasion. J Virol, 2012. 86(6): p. 2900-10.

166. Kato, H., et al., Differential roles of MDA5 and RIG-I helicases in the recognition of $R N A$ viruses. Nature, 2006. 441(7089): p. 101-5. 
167.

Hayes, M.W., et al., Pathogenic Old World arenaviruses inhibit TLR2/Maldependent proinflammatory cytokines in vitro. J Virol, 2012. 86(13): p. 7216-26.

168. Djavani, M.M., et al., Early blood profiles of virus infection in a monkey model for Lassa fever. J Virol, 2007. 81(15): p. 7960-73.

169. Rai, S.K., et al., Murine infection with lymphocytic choriomeningitis virus following gastric inoculation. J Virol, 1996. 70(10): p. 7213-8.

170. Rai, S.K., et al., Timed appearance of lymphocytic choriomeningitis virus after gastric inoculation of mice. Am J Pathol, 1997. 151(2): p. 633-9.

171. Lukashevich, I.S., et al., Hemorrhagic fever occurs after intravenous, but not after intragastric, inoculation of rhesus macaques with lymphocytic choriomeningitis virus. Journal of medical virology, 2002. 67(2): p. 171186.

172. Fogh, J., J.M. Fogh, and T. Orfeo, One hundred and twenty-seven cultured human tumor cell lines producing tumors in nude mice. J Natl Cancer Inst, 1977. 59(1): p. 221-6.

173. Hidalgo, I.J., T.J. Raub, and R.T. Borchardt, Characterization of the human colon carcinoma cell line (Caco-2) as a model system for intestinal epithelial permeability. Gastroenterology, 1989. 96(3): p. 736-749.

174. Pinto, I.M., Enterocyte-like Differentiation and Polarization of the Human Colon Carcinoma Cell Line Caco-2 in Culture. Biol. Cell, 1983. 47: p. 323330.

175. Artursson, P. and J. Karlsson, Correlation between oral drug absorption in humans and apparent drug permeability coefficients in human intestinal epithelial (Caco-2) cells. Biochem Biophys Res Commun, 1991. 175(3): p. 880-5.

176. Cheng, K.C., C. Li, and A.S. Uss, Prediction of oral drug absorption in humans--from cultured cell lines and experimental animals. Expert Opin Drug Metab Toxicol, 2008. 4(5): p. 581-90.

177. Sun, $\mathrm{H}$., et al., The Caco-2 cell monolayer: usefulness and limitations. Expert Opin Drug Metab Toxicol, 2008. 4(4): p. 395-411.

178. Sun, H., et al., A catenary model to study transport and conjugation of baicalein, a bioactive flavonoid, in the Caco-2 cell monolayer: demonstration of substrate inhibition. J Pharmacol Exp Ther, 2008. 326(1): p. 117-26.

179. Engle, M.J., G.S. Goetz, and D.H. Alpers, Caco-2 cells express a combination of colonocyte and enterocyte phenotypes. J Cell Physiol, 1998. 174(3): p. 362-9.

180. Montali, R.J., et al., A common-source outbreak of callitrichid hepatitis in captive tamarins and marmosets. J Infect Dis, 1993. 167(4): p. 946-50.

181. Montali, R.J., et al., Pathology and immunohistochemistry of callitrichid hepatitis, an emerging disease of captive New World primates caused by lymphocytic choriomeningitis virus. Am J Pathol, 1995. 147(5): p. 1441-9.

182. Rodas, J.D., et al., Mucosal arenavirus infection of primates can protect them from lethal hemorrhagic fever. J Med Virol, 2004. 72(3): p. 424-35.

183. Stephenson, E.H., E.W. Larson, and J.W. Dominik, Effect of environmental factors on aerosol-induced Lassa virus infection. J Med Virol, 1984. 14(4): p. 295-303.

184. Lukashevich, I.S., et al., [The isolation and characteristics of reassortants between the Lassa and Mopeia arenaviruses]. Vopr Virusol, 1991. 36(2): p. $146-50$. 
185. Lukashevich, I.S., Generation of reassortants between African arenaviruses. Virology, 1992. 188(2): p. 600-5.

186. Mori, A., H. Satsu, and M. Shimizu, New model for studying the migration of immune cells into intestinal epithelial cell monolayers. Cytotechnology, 2003. 43(1): p. 57-64.

187. Grasset, E., et al., Epithelial properties of human colonic carcinoma cell line Caco-2: electrical parameters. Am J Physiol, 1984. 247(3 Pt 1): p. C260-7.

188. Benda, R. and J. Cinatl, Active Immunoprophylaxis of Experimental Inhalation Lymphocytic Choriomeningitis. J Hyg Epidemiol Microbiol Immunol, 1964. 8: p. 252-61.

189. Benda, R., L. Danes, and M. Fuchsova, Experimental Inhalation Infection of Guinea-Pigs with the Virus of Lymphocytic Choriomeningitis. J Hyg Epidemiol Microbiol Immunol, 1964. 8: p. 87-99.

190. $\quad$ Oppliger, J., et al., Lassa Virus Cell Entry via Dystroglycan Involves an Unusual Pathway of Macropinocytosis. J Virol, 2016. 90(14): p. 6412-29.

191. Torriani, G., C. Galan-Navarro, and S. Kunz, Lassa Virus Cell Entry Reveals New Aspects of Virus-Host Cell Interaction. J Virol, 2017. 91(4).

192. Salvato, M., et al., Family Arenaviridae, $p$ 715-723. Virus taxonomy: ninth report of the International Committee on Taxonomy of Viruses. Academic Press, New York, NY, 2012.

193. Maes P, S.V.A., Y. Boa, M Beer, M. Birkhead, al. TBe, Taxonomy of the family ARENAVIRIDAE and the order BUNYAVIRALES: Updates 2018. Arch Virol, 2018(in press).

194. Peters, C., et al., Experimental studies of arenaviral hemorrhagic fevers, in Arenaviruses. 1987, Springer. p. 5-68.

195. Zapata, J.C., et al., Lymphocytic choriomeningitis virus (LCMV) infection of macaques: a model for Lassa fever. Antiviral Res, 2011. 92(2): p. 12538.

196. Bowen, M.D., et al., Genetic diversity among Lassa virus strains. J Virol, 2000. 74(15): p. 6992-7004.

197. Albariño, C.G., et al., High diversity and ancient common ancestry of lymphocytic choriomeningitis virus. Emerging infectious diseases, 2010. 16(7): p. 1093.

198. Safronetz, D., et al., Detection of Lassa virus, Mali. Emerging infectious diseases, 2010. 16(7): p. 1123.

199. Kouadio, L., et al., Lassa virus in multimammate rats, Côte d'lvoire, 2013. Emerging Infectious Diseases, 2015. 21(8): p. 1481.

200. Manning, J.T., N. Forrester, and S. Paessler, Lassa virus isolates from Mali and the Ivory Coast represent an emerging fifth lineage. Frontiers in microbiology, 2015. 6: p. 1037.

201. Whitmer, S.L., et al., New Lineage of Lassa Virus, Togo, 2016. Emerging infectious diseases, 2018. 24(3): p. 599.

202. LapoŠOvÁ, K., S. PastorekovÁ, and J. TomÁŠKovÁ, Lymphocytic choriomeningitis virus: invisible but not innocent. Acta virologica, 2013. 57(02): p. 160-170.

203. Price, M.E., et al., A prospective study of maternal and fetal outcome in acute Lassa fever infection during pregnancy. BMJ, 1988. 297(6648): p. 584-7. 
204. Riviere, Y., et al., Genetic mapping of lymphocytic choriomeningitis virus pathogenicity: virulence in guinea pigs is associated with the L RNA segment. J Virol, 1985. 55(3): p. 704-9.

205. Lukashevich, I.S., Advanced vaccine candidates for Lassa fever. Viruses, 2012. 4(11): p. 2514-57.

206. Russier, M., D. Pannetier, and S. Baize, Immune responses and Lassa virus infection. Viruses 4: 2766-2785. 2012.

207. Prescott, J.B., et al., Immunobiology of Ebola and Lassa virus infections. Nature Reviews Immunology, 2017. 17(3): p. 195.

208. Warner, N.L., et al., Mammarenaviral Infection Is Dependent on Directional Exposure to and Release from Polarized Intestinal Epithelia. Viruses, 2018. 10(2).

209. Hastie, K.M., et al., Crystal structure of the prefusion surface glycoprotein of the prototypic arenavirus LCMV. Nature Structural and Molecular Biology, 2016. 23(6): p. 513.

210. Goncalves, A.R., et al., Role of DC-SIGN in Lassa virus entry into human dendritic cells. J Virol, 2013. 87(21): p. 11504-15.

211. Fedeli, C., et al., Axl Can Serve as Entry Factor for Lassa Virus Depending on the Functional Glycosylation of Dystroglycan. J Virol, 2018. 92(5).

212. Beier, J.I., et al., Novel mechanism of arenavirus-induced liver pathology. PLoS One, 2015. 10(3): p. e0122839.

213. Glushakova, S.E., et al., The fusion of artificial lipid membranes induced by the synthetic arenavirus 'fusion peptide'. Biochim Biophys Acta, 1992. 1110(2): p. 202-8.

214. Iwasaki, M., Minder P, Caì Y, Kuhn JH, Yates JR III, Torbett BE, et al., Interactome analysis of the lymphocytic choriomeningitis virus nucleoprotein in infected cells reveals ATPase $\mathrm{Na}+\mathrm{K}+$ transporting subunit Alpha 1 and prohibitin as host-cell factors involved in the life cycle of mammarenaviruses. . PLoS Pathog, 2018. 4(2) p. e1006892.

215. Danthi, P. and M. Chow, Cholesterol Removal by Methyl- -Cyclodextrin Inhibits Poliovirus Entry. Journal of Virology, 2003. 78(1): p. 33-41.

216. Lambert, D., C.A. O'Neill, and P.J. Padfield, Depletion of Caco-2 cell cholesterol disrupts barrier function by altering the detergent solubility and distribution of specific tight-junction proteins. Biochem J, 2005. 387(Pt 2): p. 553-60.

217. Le Blanc, I., et al., Endosome-to-cytosol transport of viral nucleocapsids. Nat Cell Biol, 2005. 7(7): p. 653-64.

218. Cosset, F.L., et al., Characterization of Lassa virus cell entry and neutralization with Lassa virus pseudoparticles. J Virol, 2009. 83(7): p. 3228-37.

219. Iwasaki, A. and R. Medzhitov, Toll-like receptor control of the adaptive immune responses. Nature immunology, 2004. 5(10): p. 987.

220. Blasius, A.L. and B. Beutler, Intracellular toll-like receptors. Immunity, 2010. 32(3): p. 305-315.

221. Lee, B.L. and G.M. Barton, Trafficking of endosomal Toll-like receptors. Trends in cell biology, 2014. 24(6): p. 360-369.

222. Brown, J., et al., TLR-signaling networks: an integration of adaptor molecules, kinases, and cross-talk. Journal of dental research, 2011. 90(4): p. 417-427. 
223.

Kawagoe, T., et al., Sequential control of Toll-like receptor-dependent responses by IRAK1 and IRAK2. Nature immunology, 2008. 9(6): p. 684.

224. Beier, J.I., Jokinen,J.D., Gretchen E. Holz, G.E., Whang, P.S., Amah M. Martin, A.M., Warner, N.L., Arteel, G.E., Lukashevich, I.S., Novel Mechanism of Arenavirus-Induced Liver Pathology. PLoS One, 2015. 10(3): p. e0122839.

225. Glushakova, S.E. and I.S. Lukashevich, Early events in arenavirus replication are sensitive to lysosomotropic compounds. Arch Virol, 1989. 104(1-2): p. 157-61.

226. Bergthaler, A., et al., Contributions of the lymphocytic choriomeningitis virus glycoprotein and polymerase to strain-specific differences in murine liver pathogenicity. Journal of General Virology, 2007. 88(2): p. 592-603.

227. Bergthaler, A., et al., Viral replicative capacity is the primary determinant of lymphocytic choriomeningitis virus persistence and immunosuppression. Proceedings of the National Academy of Sciences, 2010. 107(50): p. 21641-21646.

228. Zhou, S., et al., Discovery of a novel TLR2 signaling inhibitor with antiviral activity. Antiviral research, 2010. 87(3): p. 295-306.

229. Cuevas, C.D., et al., Junin virus infects mouse cells and induces innate immune responses. Journal of virology, 2011. 85(21): p. 11058-11068.

230. Jung, A., et al., Lymphocytoid choriomeningitis virus activates plasmacytoid dendritic cells and induces a cytotoxic T-cell response via MyD88. Journal of virology, 2008. 82(1): p. 196-206.

231. Mercer, J. and U.F. Greber, Virus interactions with endocytic pathways in macrophages and dendritic cells. Trends in microbiology, 2013. 21(8): p. 380-388.

232. Djavani, M., et al., Mucosal immunization with Salmonella typhimurium expressing Lassa virus nucleocapsid protein cross-protects mice from lethal challenge with lymphocytic choriomeningitis virus. Journal of human virology, 2001. 4(2): p. 103.

233. Droniou-Bonzom, M.E., et al., Substitutions in the glycoprotein (GP) of the Candid\#1 vaccine strain of Junin virus increase dependence on human transferrin receptor 1 for entry and destabilize the metastable conformation of GP. J Virol, 2011. 85(24): p. 13457-62.

234. Albarino, C.G., et al., The major determinant of attenuation in mice of the Candid1 vaccine for Argentine hemorrhagic fever is located in the G2 glycoprotein transmembrane domain. J Virol, 2011. 85(19): p. 10404-8. 235. O'Hearn, A.E., et al., Serosurveillance of viral pathogens circulating in West Africa. Virol J, 2016. 13(1): p. 163.

236. Epelboin, L., et al., Is dengue and malaria co-infection more severe than single infections? A retrospective matched-pair study in French Guiana. Malar J, 2012. 11: p. 142. 


\section{CURRICULUM VITAE \\ Nikole L. Warner \\ Department of Microbiology and Immunology \\ University of Louisville School of Medicine \\ 505 South Hancock Street \\ Louisville, KY, 40202 \\ (502) 852-5394 \\ nikole.warner@louisville.edu}

\section{EDUCATION}

$08 / 2006-05 / 2010$

$08 / 2012-12 / 2014$

08/2012- May 2018
B.S. in Biology, Marian University, Fond du Lac, WI M.S. in Microbiology and Immunology, University of Louisville, Louisville, KY

Louisville, Louisville, KY

Dissertation Title: The involvement of epithelial cells in arenavirus-induced pathogenesis.

Principal Investigator: Igor S. Lukashevich

\section{ACADEMIC APPOINTMENTS}

08/2012-Present Graduate Research Assistant, Department of Microbiology and Immunology, University of Louisville, Louisville, KY

08/2017-Present Adjunct Anatomy and Physiology Professor, Ivy Tech Community College, Sellersburg, IN

\section{OTHER POSITIONS AND EMPLOYMENT}

07/2011-07/2012 Gehl Foods Inc., Quality Assurance Lab Technician, Germantown, WI

\section{PROFESSIONAL MEMBERSHIPS AND ACTIVITIES}

2007-2010 Member of the American Society for Microbiology 
2013-present Center of Predictive Medicine for Biodefense and Emerging Infectious Diseases (CPM)

2014-present Member of the American Society for Virology

\section{HONORS AND AWARDS}

08/2012-Present Integrated Programs in Biomedical Sciences Fellow, University of Louisville, Louisville, KY

$06 / 2016$

Student Travel Award, American Society for Virology Annual Meeting, Blacksburg Virginia

\section{COMMITTEE ASSIGNMENTS AND ADMINISTRATIVE SERVICES}

$\begin{array}{ll}05 / 2008-05 / 2009 & \begin{array}{l}\text { Vice President of Science and Math Association, Marian } \\ \text { University, Fond du Lac, WI }\end{array} \\ 05 / 2009-05 / 2010 & \begin{array}{l}\text { President of Science and Math Association, Marian } \\ \text { University, Fond du Lac, WI }\end{array} \\ 01 / 2015-05 / 2016 & \begin{array}{l}\text { Outreach Coordinator for Science Policy and Outreach } \\ \text { Group, University of Louisville, } \\ \text { Louisville, KY }\end{array} \\ \text { 05/2015-05/2017 } & \begin{array}{l}\text { Admissions Representative for Microbiology and } \\ \text { Immunology Student Organization (MISO), University of } \\ \text { Louisville, Louisville, KY }\end{array} \\ \text { Director of Virology Discussion Group, University of } \\ \text { Louisville, Louisville, KY }\end{array}$

\section{EDUCATIONAL ACTIVITIES}

\section{LECTURES/PRIMARY INSTRUCTION}

08/2008-05/2009 Physics Tutor, Department of Math and Natural Science, Marian University, Fond du Lac, WI

08/2009-05/2010 First-Year Studies Program Student Mentor, Marian University, Fond du Lac, WI

08/2009-12/2009 Teaching Assistant for Freshman Biology Lab, Marian University, Fond du Lac, WI

2016-2017 Graduate Teaching Academy, University of Louisville, Louisville, $\mathrm{KY}$

11/2016 MBIO 610: Research Methods in Microbiology and Immunology Lecture: In vitro virology methods, University of Louisville, Louisville, KY

01/2017 MBIO 604: General Virology Lecture, University of Louisville, Louisville, KY

11/2017 MBIO 610: Research Methods in Microbiology and Immunology Lecture: In vitro virology methods, University of Louisville, Louisville, KY 


\section{OUTREACH}

03/2015

Career Fair volunteer, Stuart Middle School,

$03 / 2015$

$07 / 2015$

$10 / 2015$

$12 / 2015$

$03 / 2016$ Louisville, KY Louisville Regional Science and Engineering Fair Judge, Louisville, KY GEAR UP Camp, Volunteer leader and speaker, Louisville, KY

PULSE day at the Kentucky Science Center, Volunteer leader and speaker, Louisville, KY Student Mentoring for Louisville Science Fair Students, Louisville, KY Louisville Regional Science and Engineering Fair Judge, Louisville, KY

\section{ABSTRACTS AND PRESENTATIONS}

\section{ORAL PRESENTATIONS}

\section{Local/Regional}

1. Anders LC, Douglas AN, Mohammad MK, Massey VL, Kirpich IA, Falkner KC, Warner NL, Arteel GE, Cave M, McClain CJ and Beier JI (2014) Vinyl Chloride Predisposes The Liver To Injury: Implications For Human Exposure. Gastroenterology 146(5):S910. (Selected for oral presentation).

2. Warner NL (2013) The Effect of Cell Polarity on Viral Infection of Hemorrhagic Fever Viruses. Research mini seminar. Virology Research Discussion, Department of Microbiology and Immunology, University of Louisville, Louisville, KY

3. Warner NL (2014) Polarized Epithelial Cells in Mammarenavirus Pathogenicity. Research mini seminar. Virology Research Discussion, Department of Microbiology and Immunology, University of Louisville, Louisville, KY

4. Warner NL (2014) Polarized Epithelial Cells in Mammarenavirus Pathogenicity. Department of Microbiology and Immunology Seminar, University of Louisville, Louisville, KY

5. Warner NL (2015) Polarized Epithelial Cells in Mammarenavirus Pathogenicity. Center for Predictive Medicine Bi-annual Retreat, University of Louisville, Louisville, KY 
6. Warner, NL (2016) The Interaction of Mammarenaviruses with Polarized Epithelial Cells. Department of Microbiology and Immunology Seminar, University of Louisville, Louisville, KY

7. Warner NL (2016) Polarized Epithelial Cells in Mammarenavirus Pathogenicity. Center for Predictive Medicine Bi-annual Retreat, University of Louisville, Louisville, KY

8. Warner NL (2016) Polarized Epithelial Cells in Mammarenavirus Pathogenicity. Research mini seminar. Virology Research Discussion, Department of Microbiology and Immunology, University of Louisville, Louisville, KY

9. Warner, NL (2017) The Interaction of Mammarenaviruses with Polarized Epithelial Cells. Department of Microbiology and Immunology Seminar, University of Louisville, Louisville, KY

\section{POSTERS}

\section{National/International}

1. Holz GE, Beier JI, Jokinen JD, Warner NL, Arteel GE, Lukashevich IS. 2015. Arenavirus-induced hepatic cell cycle arrest. American Society for Virology Annual Meeting, Western University, London, Ontario, Canada.

2. Poole LG, Massey VL, Torres E, Falkner KC, Siow DL, Warner NL, Schmidt RH, Ritzenthaler JD, Roman J, Arteel GE. (2015) TNF $\alpha$ mediates the liver:lung axis in alcohol-enhanced acute lung injury in mice. The Liver Meeting, San Francisco CA

3. Poole LG, Massey VL, Torres E, Siow DL, Warner NL, Lang AL, Dolin CE, Ritzenthaler J, Roman J, Arteel GE. (2015) Chronic ethanol exposure sensitizes the lung in a mouse model of endotoxemia-induced acute lung injury: potential role of plasminogen activator inhibitor-1. Society for Leukocyte Biology Annual Meeting, Raleigh NC

4. Warner NL, Jokinen JD, Arteel GE, Lukashevich IS (2016). Interaction of arenaviruses with polarized epithelial cells. The American Society of Virology $25^{\text {th }}$ Annual Meeting. Blacksburg, VA.

5. Holz GE, Jokinen JD, Warner NL, Chung DH, Beier JI, Arteel GE, Lukashevich IS. 2016. Arenavirus-induced disruption of cell cycle progression of hepatocytes and liver pathology. Keystone Symposia Series: Hemorrhagic Fever Viruses, Santa Fe, New Mexico.

6. Poole LG, Beier JI, Torres-Gonzáles E, Anwar-Mohamed A, Warner NL, Dolin CE, Nguyen-Ho CT, Roman J, Arteel GE. 2016. Acute-on-chronic alcohol exposure using the 'NIAAA model' concomitantly damages the liver and lung. The Liver Meeting, Boston MA 
7. Poole LG, Massey VL, Torres-Gonzáles E, Warner NL, Siow DL, Luyendyk JP, Dolin CE, Ritzenthaler J, Roman J, Arteel GE. 2016. Plasminogen activator inhibitor-1 plays a critical role in alcoholenhanced acute lung injury. American Thoracic Society, San Francisco, CA

\section{Local/Regional}

1. Warner NL, Beier JI, Jokinen JD, Holz GE, Arteel GE, Lukashevich IS (2013) Modification of epithelial cell monolayer integrity increases apical infectivity to lymphocytic choriomeningitis virus (LCMV) in Caco-2 cells. Research! Louisville.

2. Warner NL, Jokinen J, Arteel GE, Lukashevich IS (2014) Interaction of arenaviruses with polarized Caco-2 cells. Reasearch!Louisville

3. Holz G, Jokinen J, Warner NL, Arteel GE, Lukashevich IL (2015) Arenavirus-induced liver pathology: search for biomarkers of liver involvement in in vitro models. Research!Louisville

4. Warner NL, Jokinen J, Holz GE, Arteel GE, Lukashevich IL (2015) Interaction of arenaviruses with polarized epithelial cells. Research!Louisville

5. Poole LG, Massey VL, Torres E, Siow DL, Warner NL, Lang AL, Dolin CE, Ritzenthaler J, Roman J, Arteel GE. (2015) Chronic ethanol exposure sensitizes the lung in a mouse model of endotoxemia-induced acute lung injury: potential role of plasminogen activator inhibitor-1. Research!Louisville

6. Warner NL, Jokinen J, Arteel GE, Lukashevich IL (2016) The Role of Epithelial Cell Polarity in Mammarenavirus Infections in Vivo. Research!Louisville

7. Holz GE, Jokinen JD, Warner NL, Chung DH, Lukashevich IS. 2016. TNF-a stimulation in LCMV-infected hepatic in vitro model promotes viral replication and upregulates cell cycling machinery. Research!Louisville, Louisville. KY.

8. Poole LG, Beier JI, Torres E, Mohamed A, Warner NL, Dolin CE, Nguyen-Ho CT, Ritzenthaler JD, Roman J, Arteel GE. 2016. Acute-onchronic alcohol exposure using the 'NIAAA model' concomitantly damages the liver and lung. Research!Louisville, Louisville, KY.

9. Poole LG, Beier JI, Torres E, Mohamed A, Warner NL, Dolin CE, Nguyen-Ho CT, Ritzenthaler JD, Roman J, Arteel GE. 2016. Acute-onchronic alcohol exposure using the 'NIAAA model' concomitantly damages the liver and lung. Ohio Valley Society of Toxicology Summer Meeting, Cincinnati $\mathrm{OH}$

10. Warner NL, Jokinen JD, Lukashevich IS. (2017). Research! Louisville Replication of OW Mammarenaviruses in Polarized Epithelial Cells. Research! Louisville. University of Louisville, Louisville, KY. 


\section{ABSTRACTS}

\section{National/International}

1. Warner NL, Kirpich IA, Mohammad MK, Falkner KC, Cave M, McClain CJ and Beier JI (2013) Vinyl Chloride And/Or Its Metabolites Induce Hepatic Necro-Inflammation In Mice And In Human Subjects With Chronic Low-Level Exposures. Hepatology 58:949A.

2. Holz GE, Beier JI, Jokinen JD, Warner NL, Arteel GE, Lukashevich IS. 2015. Arenavirus-induced hepatic cell cycle arrest. American Society for Virology Annual Meeting, Western University, London, Ontario, Canada.

3. Poole LG, Massey VL, Torres E, Falkner KC, Siow DL, Warner NL, Schmidt RH, Ritzenthaler JD, Roman J, Arteel GE (2015) TNFa mediates the liver:lung axis in alcohol-enhanced acute lung injury in mice. Hepatology in press

4. Poole LG, Massey VL, Torres E, Siow DL, Warner NL, Lang AL, Dolin CE, Ritzenthaler J, Roman J, Arteel GE. (2015) Chronic ethanol exposure sensitizes the lung in a mouse model of endotoxemia-induced acute lung injury: potential role of plasminogen activator inhibitor-1. Society for Leukocyte Biology Annual Meeting. Raleigh NC

5. Warner NL, Jokinen JD, Arteel GE, Lukashevich IS (2016). Interaction of arenaviruses with polarized epithelial cells. The American Society of Virology $25^{\text {th }}$ Annual Meeting. Blacksburg, VA.

6. Holz GE, Jokinen JD, Warner NL, Chung DH, Beier JI, Arteel GE, Lukashevich IS. 2016. Arenavirus-induced disruption of cell cycle progression of hepatocytes and liver pathology. Keystone Symposia Series: Hemorrhagic Fever Viruses, Santa Fe, New Mexico.

7. Poole LG, Beier JI, Torres-Gonzáles E, Anwar-Mohamed A, Warner NL, Dolin CE, Nguyen-Ho CT, Roman J, Arteel GE. Acute-on-chronic alcohol exposure using the 'NIAAA model' concomitantly damages the liver and lung. (2016) Hepatology 64 S1 (1221).

8. Poole LG, Massey VL, Torres-Gonzalez E, Siow DL, Warner NL, Luyendyk JP, Ritzenthaler JD, Roman J, Arteel GE. Plasminogen Activator Inhibitor-1 (PAI-1) Plays a Critical Role in Alcohol-Enhanced Acute Lung Injury [abstract]. Am J Respir Crit Care Med 2016:A2647.

\section{Local/Regional}

1. Warner NL, Beier JI, Jokinen JD, Holz GE, Arteel GE, Lukashevich IS (2013) Modification of epithelial cell monolayer integrity increases apical infectivity to lymphocytic choriomeningitis virus (LCMV) in Caco-2 cells. Research!Louisville. 
2. Anders LC, Douglas AN, Warner NL, Kirpich IA, Mohammad MK, Falkner KC, Cave M, McClain CJ and Beier JI (2013) Vinyl Chloride And/Or Its Metabolites Induce Hepatic Necro-Inflammation In Mice And In Human Subjects With Chronic Low-Level Exposures. Research! Louisville.

3. Holz GE, Beier JI, Jokinen JD, Warner NL, Arteel GE, Lukashevich IS (2013) Design of tissue culture model to study interaction between macrophages and hepatocytes in Arenavirus-induced liver pathology. Research!Louisville.

4. Whang PS, Beier JI, Jokinen JD, Martin AM, Warner NL, Arteel GE and Lukashevich IS (2013) A Novel Mechanism of Arenavirus-induced Liver Pathology. Research!Louisville.

5. Warner NL, Jokinen J, Arteel GE, Lukashevich IS (2014) Interaction of arenaviruses with polarized Caco-2 cells. Reasearch!Louisville

6. Holz G, Jokinen J, Warner NL, Arteel GE, Lukashevich IL (2015) Arenavirus-induced liver pathology: search for biomarkers of liver involvement in in vitro models. Research!Louisville

7. Warner NL, Jokinen J, Holz GE, Arteel GE, Lukashevich IL (2015) Interaction of arenaviruses with polarized epithelial cells. Research!Louisville

8. Poole LG, Massey VL, Torres E, Siow DL, Warner NL, Lang AL, Dolin CE, Ritzenthaler J, Roman J, Arteel GE. (2015) Chronic ethanol exposure sensitizes the lung in a mouse model of endotoxemia-induced acute lung injury: potential role of plasminogen activator inhibitor-1. Research!Louisville

9. Warner NL, Jokinen J, Arteel GE, Lukashevich IL (2016) The Role of Epithelial Cell Polarity in Mammarenavirus Infections in Vivo. Research!Louisville

10. Holz GE, Jokinen JD, Warner NL, Chung DH, Lukashevich IS. 2016. TNF- $\alpha$ stimulation in LCMV-infected hepatic in vitro model promotes viral replication and upregulates cell cycling machinery. Research!Louisville, Louisville. KY.

11. Poole LG, Beier JI, Torres-Gonzáles E, Anwar-Mohamed A, Warner NL, Dolin CE, Nguyen-Ho CT, Roman J, Arteel GE. (2016). Concomitant Liver and Lung Injury in the "NIAAA Model" of Chronic + Binge Alcohol Exposure. Ohio Valley Society of Toxicology Annual Meeting, Indianapolis, IN.

12. Poole LG, Beier JI, Torres-Gonzáles E, Anwar-Mohamed A, Warner NL, Dolin CE, Nguyen-Ho CT, Roman J, Arteel GE. (2016). Acute-onchronic alcohol exposure using the 'NIAAA model' concomitantly damages the liver and lung. Research!Louisville, Louisville, KY.

13. Poole LG, Beier JI, Torres-Gonzáles E, Anwar-Mohamed A, Warner NL, Dolin CE, Nguyen-Ho CT, Roman J, Arteel GE. (2016). Acute-onchronic alcohol exposure using the 'NIAAA model' concomitantly 
damages the liver and lung. Ohio Valley Society of Toxicology Summer Meeting, Cincinnati, $\mathrm{OH}$.

14. Warner NL, Jokinen JD, Lukashevich IS. (2017). Research! Louisville Replication of OW Mammarenaviruses in Polarized Epithelial Cells. Research! Louisville. University of Louisville, Louisville, KY.

\section{PUBLICATIONS}

\section{PEER-REVIEWED}

1. Beier JI, Jokinen JD, Holz GE, Whang PS, Martin AM, Warner NL, Arteel GE, Lukashevich IS. (2015) Novel mechanism of arenavirusinduced liver pathology. PLoS One 10: e0122839.

2. Massey VL, Poole LG, Siow DL, Torres E, Warner NL, Schmidt RH, et al. Chronic Alcohol Exposure Enhances Lipopolysaccharide-Induced Lung Injury in Mice: Potential Role of Systemic Tumor Necrosis FactorAlpha. Alcohol Clin Exp Res. 2015;39(10):1978-88. doi:

10.1111/acer.12855. PubMed PMID: 26380957.

3. Anders LC, Lang AL, Anwar-Mohamed A, Douglas AN, Bushau AM, Falkner KC, Hill BG, Warner NL, et al. Vinyl Chloride Metabolites Potentiate Inflammatory Liver Injury Caused by LPS in Mice. Toxicological sciences : an official journal of the Society of Toxicology. 2016;151(2):312-23. Epub 2016/03/11. doi: 10.1093/toxsci/kfw045. PubMed PMID: 26962056; PubMed Central PMCID: PMCPmc4880135.

4. Poole LG, Massey VL, Siow DL, Torres-Gonzalez E, Warner NL, Luyendyk JP, et al. Plasminogen Activator Inhibitor-1 is Critical in Alcohol-enhanced Acute Lung Injury in Mice. American journal of respiratory cell and molecular biology. 2017. Epub 2017/04/27. doi: 10.1165/rcmb.2016-0184OC. PubMed PMID: 28445073.

5. Warner NL, Jokinen JD, Beier JI, Sokoloski KJ, Lukashevich IS. Mammarenaviral Infection Is Dependent on Directional Exposure to and Release from Polarized Intestinal Epithelia. Viruses. 2018;10(2). doi: 10.3390/v10020075. PubMed PMID: 29439402.

\section{${ }^{\star}$ Featured on the cover of February Edition}

6. Poole LG, Beier JI, Torres-Gonzáles E, Nguyen-Ho CT, Schlueter CF, Hoyle GW, Warner NL, Dolin CE, Roman J, Arteel GE. Chronic + binge alcohol exposure promotes inflammation and alters airway mechanics in the lunkg. Alcohol. 2018. Under Review.

7. Wang, $\mathrm{M}^{\#}$, Warner NL\#, Jokinen, JD, Lukashevich, IS. Strain-specific intracellular trafficking of LCMV, a prototypic mammalian arenavirus. Viruses 2018. Under Review. \#denotes co-first authorship 